\title{
THERMOPHYSICAL PROPERTIES
}

\author{
Quarterly Report
}

1 October 1992 - 31 December 1992

Richard F. Kayser

\section{Thymophysics Division}

Building 221, Room A 105

National Institute of Standards and Technology

Gaithersburg, Maryland 20899

January 1993

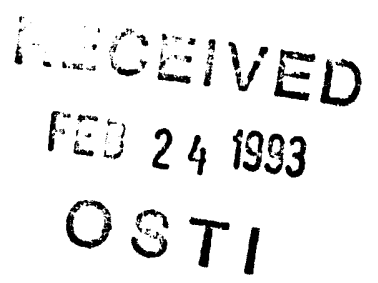

Prepared for

The Air-Conditioning and Refrigeration Technology Institute Under

ARTI MCLR Project Number 650-50800

This research project is supported, in whole or in part, by U.S. Department of Energy grant number DE-FG0291CE23810: Materials Compatibility and Lubricants Research (MCLR) on CFC-Refrigerant Substitutes. Federal funding supporting this project constitutes $93.67 \%$ of allowable costs. Funding from non-government sources supporting this project consists of direct cost sharing of $6.33 \%$ of allowable costs; and in-kind contributions from the air-conditioning and refrigeration industry. 


\section{DISCLAIMER}

The U.S. Department of Energy's and the air-conditioning industry's support for the Materials Compatibility and Lubricants Research (MCLR) program does not constitute an endorsement by the U.S. Department of Energy, nor by the air-conditioning and refrigeration industry, of the views expressed herein.

\section{NOTICE}

This report was prepared on account of work sponsored by the United States Government. Neither the United States Government, nor the Department of Energy, nor the Air Conditioning and Refrigeration Technology Institute, nor any of their employees, nor any of their contractors, subcontractors, or their employees, makes any warranty, expressed or implied, or assumes any legal liability or responsibility for the accuracy, completeness, or usefulness of any information, apparatus, product or process disclosed or represents that its use would not infringe privatelyowned rights.

\section{COPYRIGHT NOTICE}

(for journal publication submissions)

By acceptance of this article, the publisher and/or recipient acknowledges the right of the U.S. Government and the Air-Conditioning and Refrigeration Technology Institute, Inc. (ARTI) to retain a nonexclusive, royalty-free license in and to any copyrights covering this paper. 
DOE/CE/23810-8A

\title{
THERMOPHYSICAL PROPERTIES
}

\author{
ARTI MCLR Project Number 650-50800
}

\author{
Richard F. Kayser \\ Thermophysics Division \\ National Institute of Standards and Technology
}

\begin{abstract}
Numerous fluids have been identified as promising alternative refrigerants, but much of the information needed to predict their behavior as pure fluids and as components in mixtures does not exist. In particular, reliable thermophysical properties data and models are needed to predict the performance of the new refrigerants in heating and cooling equipment and to design and optimize equipment to be reliable and energy efficient. The objective of this project is to provide highly accurate, selected thermophysical properties data for Refrigerants $32,123,124$, and 125 , and to use these data to fit simple and complex equations of state and detailed transport property models. The new data will fill gaps in the existing data sets and resolve the problems and uncertainties that exist in and between the data sets. This report describes the prugress made during the fourth quarter of this fifteen-month project, which was initiated in late January, 1992.
\end{abstract}

\section{SCOPE}

This project involves selected measurements of the thermodynamic properties of HFC-32, HCFC-124, and HFC-125, and the development of high-accuracy modified Benedict-WebbRubin (MBWR) equations of state and improved Carnahan-Starling-DeSantis (CSD) equations of state for each fluid. It also includes selected measurements of the transport properties of HFC-32 (viscosity and thermal conductivity) and HCFC-123 (thermal conductivity) and the development of detailed correlations for same. The experimental thermodynamic measurements will include, as appropriate, accurate determinations of the critical temperature, pressure, and density; vapor pressures and saturated liquid densities; ideal-gas heat capacity from measurements of the vapor-phase speed of sound; the pressure-volume-temperature (PVT) behavior in the superheated vapor region; the PVT behavior of the compressed liquid; and seiected measurements of the liquid-phase heat capacity. The experimental transport measurements will cover the one-phase and saturated liquid and vapor states over the temperature range of interest. Efforts prior to this quarter were focused mostly on measurements of the vapor- and liquid-phase PVT behavior, vapor pressure, coexisting densities, isochoric heat capacity, and transport properties of HFC-32; the vapor pressure, coexisting densities, and speed of sound of HCFC-124; the vapor pressure, coexisting densities, and isochoric heat capacity of HFC-125; and the thermal conductivity of HCFC-123. Efforts during this quarter were focused mostly on completing measurements of the vapor pressure, isochoric heat capacity, supercritical thermal conductivity, and liquid-phase viscosity of HFC-32; 
completing measurements of the low-temperature vapor pressure of HCFC-124; performing Burnett measurements of the vapor-phase PVT and high-temperature vapor pressure of HCFC124; and completing measurements of the compressed liquid density and low-temperature vapor pressure of HFC-125.

\section{SIGNIFICANT RESULTS}

HFC-32

As reported previously, the Burnett apparatus has been used in the isochoric mode to determine the PVT relation for the vapor phase. Eleven isochores were completed spanning the ranges 268 to $373 \mathrm{~K}\left(23\right.$ to $\left.212^{\circ} \mathrm{F}\right)$ and 0.018 to 1.3 times the critical density $\left(7.5\right.$ to $550 \mathrm{~kg} / \mathrm{m}^{3} ; 0.47$ to $\left.34.3 \mathrm{Ib} / \mathrm{ft}^{3}\right)$; the highest absolute pressure was $9.7 \mathrm{MPa}(1400 \mathrm{psi})$. Two Burnett expansions were completed at $373 \mathrm{~K}\left(212^{\circ} \mathrm{F}\right)$ to establish the densities of the isochores. These 147 gasphase data have now been analyzed, and the results are given in both SI and PI units in Table 1 (see Appendix A, which includes all tables).

In addition to the Burnett measurements, the vibrating tube densimeter was used previously to determine the PVT relation for the liquid phase. Twenty-one isotherms were completed spanning the ranges 243 to $343 \mathrm{~K}\left(-22\right.$ to $\left.158^{\circ} \mathrm{F}\right)$ and 2000 to $6500 \mathrm{kPa}$ (290 to $\left.940 \mathrm{psi}\right)$. These 654 liquid-phase data have now been analyzed, and the results are given in Table 2.

The Burnett apparatus has been used to measure the vapor pressure of HFC-32 at 18 temperatures in the range from $268 \mathrm{~K}\left(23^{\circ} \mathrm{F}\right)$ to the critical temperature at $351.36 \mathrm{~K}\left(172.78^{\circ} \mathrm{F}\right)$. These new vapor-pressure data are given in Table 3. As reported previously, an ebulliometer was used to measure the vapor pressure of HFC-32 at low temperatures in the range between 208 and $237 \mathrm{~K}\left(-85\right.$ and $\left.-32^{\circ} \mathrm{F}\right)$. A manuscript reporting these data has been accepted for publication in the Journal of Chemical and Engineering Data. The NIST Burnett and ebulliometric vapor-pressure data for HFC-32 have been correlated and their deviations from the resulting correlation are shown in Figure 1. Also shown in Figure 1 are the HFC-32 data of P.F. Malbrunot, et al. [J. Chem. \& Eng. Data 13, 16 (1968)]. The uncertainties in the NIST measurements are of order $0.05 \%$.

The saturated vapor and liquid densities have been obtained by extrapolating the Burnett vaporphase and vibrating tube liquid-phase PVT data to the vapor pressure curve. The saturated vapor and liquid densities so obtained are given in Tables 4 and 5, respectively.

An adiabatic calorimeter has been used to measure the molar heat capacity at constant volume $\left\{\mathrm{C}_{\mathrm{v}}\right\}$ for HFC-32 along five additional isochores, thereby extending the measurements to eight isochores. In total, $79 \mathrm{C}_{\mathrm{v}}$ values were measured in the liquid state and 105 values were measured in the vapor + liquid two-phase region. The sample purity was 0.9994 mole fraction. The temperatures ranged from 141 to $342 \mathrm{~K}\left(-206\right.$ to $\left.156^{\circ} \mathrm{F}\right)$ with pressures to $35 \mathrm{MPa}(5000$ psi). Figure 2 illustrates the pressure and temperature range covered by this study, Figure 3 shows the liquid heat capacity data as a function of temperature, and Figure 4 gives the saturated liquid heat capacity, $\mathrm{C}_{\sigma}$, derived from the two-phase measurements. The measured values are given in Tables 6 through 13 for the liquid phase and in Tables 14 through 16 for the two-phase 
region. These values are preliminary and are subject to change by as much as $\pm 1 \%$ when an accurate equation of state becomes available. In addition to the temperature-density-pressure ( $\mathrm{T}$ $\rho$-P) state conditions, the tables present estimated uncertainties of the measurements. Provided that an accurate equation of state is available for data reduction, the uncertainty of the heat capacity measurements is estimated to be $\pm 0.5 \%$.

The transient hot-wire study of the thermal conductivity of HFC-32 has been extended to include two supercritical isotherms at 365 and $380 \mathrm{~K}\left(197\right.$ and $\left.225^{\circ} \mathrm{F}\right)$. The thermal conductivity surface now includes 1926 data points over the region from 160 to $380 \mathrm{~K}\left(-167\right.$ to $\left.225^{\circ} \mathrm{F}\right)$ at pressures to $70 \mathrm{MPa}(10,000 \mathrm{psi})$. The data from 160 to $340 \mathrm{~K}\left(-167\right.$ to $\left.152^{\circ} \mathrm{F}\right)$ were provided in the last progress report. A plot of the entire thermal conductivity surface is provided for reference in Figure 5. The thermal conductivity critical enhancement is clearly visible in the two supercritical isotherms.

Measurements of the thermal conductivity of HFC- 32 at $400 \mathrm{~K}\left(260^{\circ} \mathrm{F}\right)$ were in progress when 3 of the 4 leads to the bottom of the hot wires failed. These leads were constructed of 0.003 " diameter tantalum wire. When the cell was disassembled, a reddish-brown deposit was found coating the platinum hot wires and the 316 stainless steel cell walls. The tantalum leads were extremely corroded and quite brittle; they may have experienced some type of fluorine corrosion. A chemical analysis is planned of the fluid sample and reddish-brown deposit that were taken from the cell. The transient hot-wire cell has been rebuilt using copper leads instead of the tantalum leads. The new isotherms above $340 \mathrm{~K}\left(152^{\circ} \mathrm{F}\right)$ must be rechecked with the rebuilt cell since it is not known at what temperature the corrosion became a significant problem and whether it affected the thermal conductivity data. For this reason the new supercritical data points are not provided in this report.

Measurements of the shear viscosity of compressed fluid HFC-32 have been completed at temperatures between 150 and $315 \mathrm{~K}\left(-190\right.$ to $\left.116^{\circ} \mathrm{F}\right)$ and at pressures to $30 \mathrm{MPa}(4400 \mathrm{psi})$. At small molar volumes, the fluidity (reciprocal viscosity) increases linearly with molar volume, as indicated in Figure 6, with deviations as indicated in Figure 7. Most of the data of Phillips and Murphy [J. Chem. \& Eng. Data 15, 304 (1970)] and of Ripple and Matar (NIST Gaithersburg, 1992) differ from the present data by less than the combined experimental errors, as shown in Figures 8 and 9, respectively. Additional measurements at temperatures to $420 \mathrm{~K}$ $\left(297^{\circ} \mathrm{F}\right)$ and pressures to $50 \mathrm{MPa}(7300 \mathrm{psi})$ are in progress.

\section{HCFC-124}

The vibrating tube densimeter was used previously to determine the PVT relation for HCFC-124 in the liquid phase. Twenty-two isotherms were completed spanning the ranges 275 to $372 \mathrm{~K}$ $\left(-35\right.$ to $\left.210^{\circ} \mathrm{F}\right)$ and 396 to $6500 \mathrm{kPa}(57$ to $922 \mathrm{psi})$. These liquid-phase data have now been analyzed, and the results are given in Table 17.

An ebulliometer has been used to measure the vapor pressure of $\mathrm{HCFC}-124$ at 44 temperatures between 222 and $286 \mathrm{~K}\left(-60\right.$ and $\left.55^{\circ} \mathrm{F}\right)$; the corresponding pressures ranged from 14 to $259 \mathrm{kPa}$ ( 2 to $37.5 \mathrm{psi})$. The new vapor-pressure data are very precise, with a standard deviation of $0.014 \%$. These data are presented in Table 18, and the results of an Antoine equation fit are 
given in Table 19. The vapor-pressure data and Antoine equation are plotted versus temperature in Figure 10, along with the deviations of the vapor-pressure data from the Antoine equation.

Burnett measurements of the gas-phase PVT behavior of HCFC-124 and of the vapor pressure at higher temperatures are in progress; approx imately 150 data have been obtained between 278 and $423 \mathrm{~K}\left(41\right.$ and $\left.302^{\circ} \mathrm{F}\right)$. These measurements will be completed by the end of January 1993.

\section{HFC-125}

The vibrating tube densimeter was used previously to determine the PVT relation for HFC-125 in the liquid phase. Fifteen isotherms were completed spanning the ranges 275 to $369 \mathrm{~K}$ (36 to $205^{\circ} \mathrm{F}$ ) and 1500 to $6200 \mathrm{kPa}(230$ to $900 \mathrm{psi})$. These liquid-phase data have now been analyzed, and the results are given in Table 20.

An isochoric PVT apparatus has been used to measure the density of compressed liquid HFC125. The sample purity is 0.9973 mole fraction. The measured temperatures ranged from 174 to $398 \mathrm{~K}\left(-146\right.$ to $\left.257^{\circ} \mathrm{F}\right)$ with pressures to $35 \mathrm{MPa}(5000 \mathrm{psi})$. Densities were measured at 87 liquid-state conditions, as shown in Figure 11. The pressures, temperatures, and densities are presented in Table 21 . The uncertainty of the densities is estimated to be $\pm 0.1 \%$.

An ebulliometer has been used to measure the vapor pressure of HFC-125 at 41 temperatures between 219 and $247 \mathrm{~K}\left(-65\right.$ and $\left.-15^{\circ} \mathrm{F}\right)$; the corresponding pressures ranged from 74 to $262 \mathrm{kPa}$ (10.7 to $38 \mathrm{psi})$. The new vapor-pressure data are very precise, with a standard deviation of $0.01 \%$. These data are presented in Table 22, and the results of an Antoine equation fit are given in Table 23. The vapor-pressure data and Antoine equation are plotted versus temperature in Figure 12, along with the deviations of the vapor-pressure data from the Antoine equation.

Burnett measurements of the gas-phase PVT behavior of HFC-125 and of the vapor pressure of HFC-125 at higher temperatures will be initiated when the work on HCFC-124 is complete.

A preliminary MBWR equation of state for HFC-125 has been developed during the current reporting period. It is valid at temperatures from 200 to $400 \mathrm{~K}\left(-100\right.$ to $\left.260^{\circ} \mathrm{F}\right)$, and it appears to be reasonable upon extrapolation to $500 \mathrm{~K}\left(440^{\circ} \mathrm{F}\right)$; the maximum pressure is $20 \mathrm{MPa}(3000$ psi). Although this equation was developed for another sponsor and can not be released until approved by the sponsor, this work will greatly speed up the MBWR fit to be done for ARTI. The existing data for HFC-125 have been put into computer files in the form required by the fitting program, and preliminary evaluations of the data have been performed. The process of fitting this equation has revealed a number of gaps and inconsistencies in the existing data and has confirmed the need for the additional measurements on HFC-125 that are being carried out under the current project.

HCFC-1̂23

The analysis of the HCFC-123 thermal conductivity data is proceeding and will be based on the new 32-term MBWR equation of state developed recently at NIST by McLinden, et al. (see below). The thermal conductivity data set includes 1618 transient hot-wire data points from 180 
to $480 \mathrm{~K}\left(-130\right.$ to $\left.405^{\circ} \mathrm{F}\right)$ at pressures to $70 \mathrm{MPa}(10,000 \mathrm{psi})$. Preliminary data points were provided in the previous progress report based on densities and heat capacities obtained from NIST corresponding-states predictions. A plot of the entire thermal conductivity surface is provided for reference in Figure 13. The thermal conductivity critical enhancement is clearly visible in the supercritical isotherm, as was the case for HFC-32.

An isochoric PVT apparatus has been used to measure the density of compressed liquid HCFC123 using the same cylinder of sample as used in a study of $\mathrm{C}_{\mathrm{v}}$ (see below). The temperatures ranged from 176 to $380 \mathrm{~K}\left(-143\right.$ to $\left.224^{\circ} \mathrm{F}\right)$ with pressures to $35 \mathrm{MPa}(5000 \mathrm{psi})$. Densities were measured at 105 liquid-state conditions. The pressures, temperatures, and densities are presented in Table 24. The uncertainty of the densities is estimated to be $\pm 0.1 \%$.

An adiabatic calorimeter has been used to measure the molar heat capacity at constant volume, $\mathrm{C}_{\mathrm{v}}$. The measurements were carried out at 79 single-phase-liquid states and 92 saturated-liquid states. Six isochores were completed on a sample of 0.9999 mole fraction purity. The temperatures ranged from 167 to $341 \mathrm{~K}\left(-159\right.$ to $\left.155^{\circ} \mathrm{F}\right)$ with pressures to $35 \mathrm{MPa}(5000 \mathrm{psi})$. The measurements for the liquid phase are presented in Tables 25 through 30 and for the twophase region in Tables 31 and 32. These values are preliminary and are subject to change by as much as $\pm 1 \%$.

The MBWR equation of state for HCFC-123 has been revised and considerably improved using the liquid-phase PVT and isochoric heat capacity data described above. This work was prompted by an evaluation of the equation of state for HCFC-123 carried out by Annex 18 of the International Energy Agency. Preliminary results of this evaluation revealed weaknesses in some of the derived properties (specifically, sound speed and heat capacity) for the previous HCFC-123 MBWR fit. The new equation is accurate for all thermodynamic properties from just above the triple point to about $550 \mathrm{~K}\left(530^{\circ} \mathrm{F}\right)$ and at pressures to $40 \mathrm{MPa}(6000 \mathrm{psi})$. Although not part of the ARTI work, this equation is needed to analyze the completed thermal conductivity measurements (see above), and it should be of great interest to many ARI members. It is available upon request.

\section{COMPLIANCE WITH AGREEMENT}

NIST has complied with all terms of the grant agreement during the fourth quarter of calendar year 1992 modulo small shifts in the estimated level of effort from one property and/or fluid to another.

\section{PRINCIPAL INVESTIGATOR EFFORT}

Dr. Richard F. Kayser is the NIST Principal Investigator for the MCLR program. During the fourth quarter of calendar year 1992, Dr. Kayser devoted approximately one week to monitoring and reviewing the research, and preparing the quarterly report. The project involves multiple researchers and capabilities in Gaithersburg, MD and Boulder, CO. 

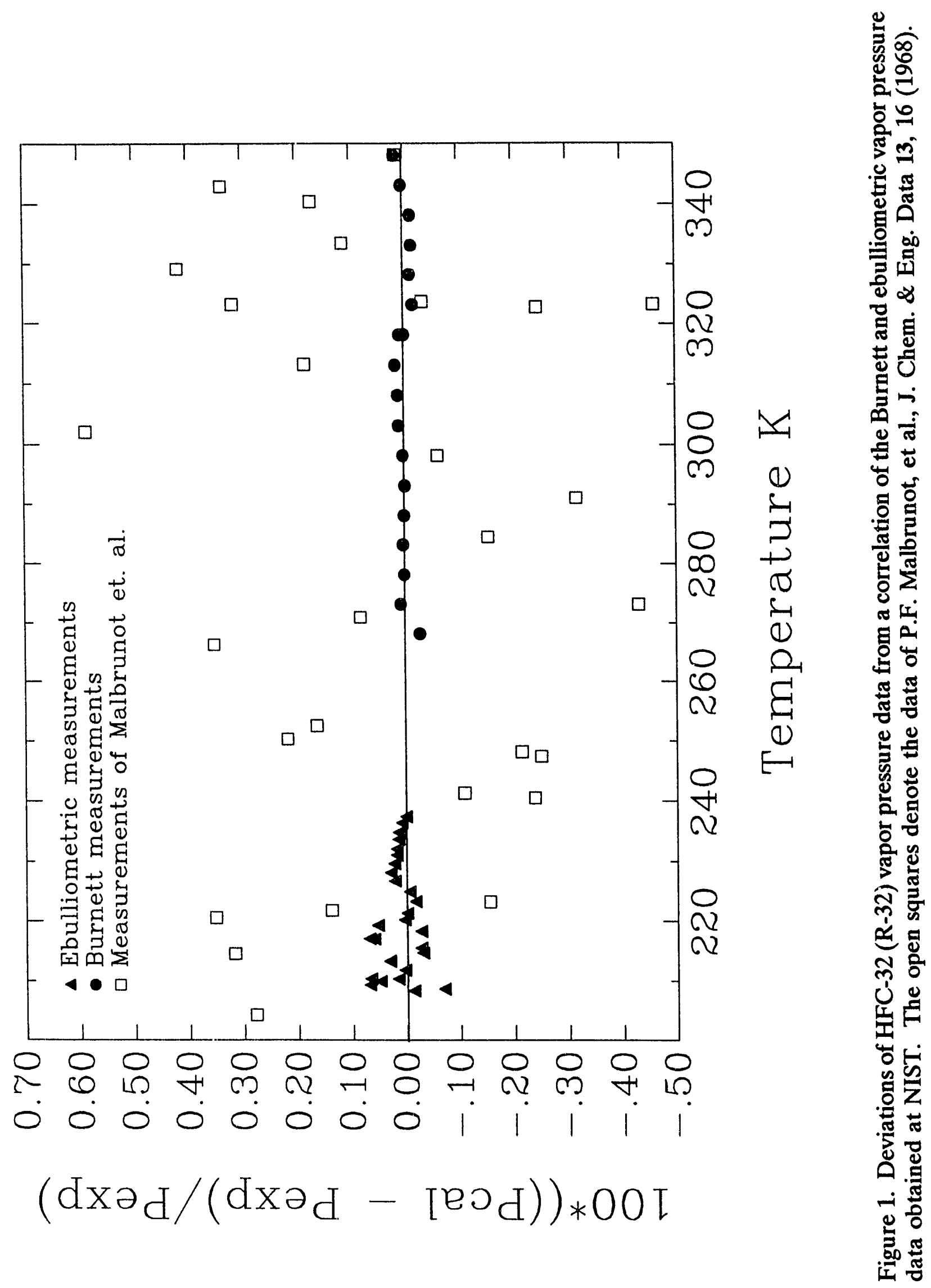


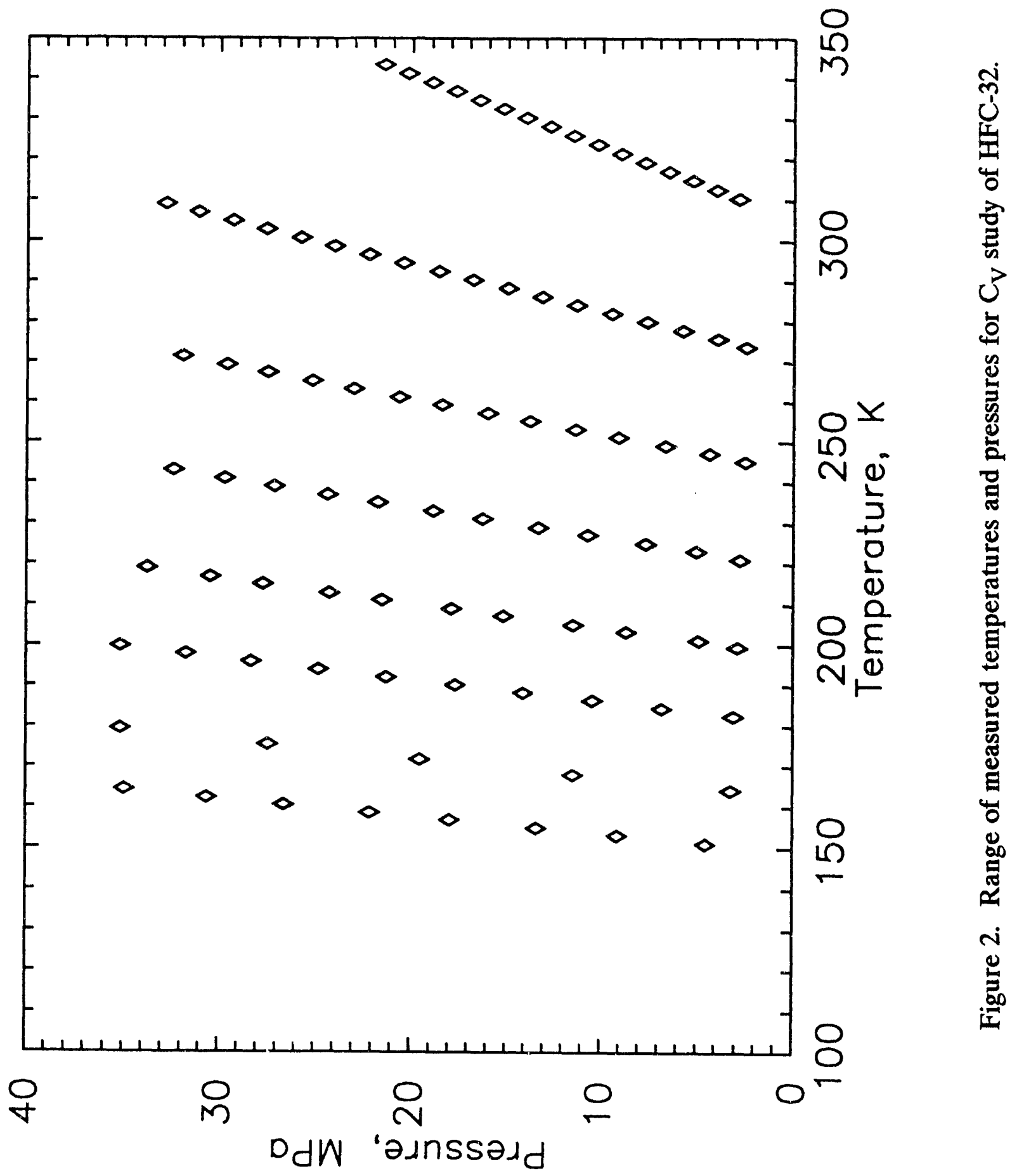




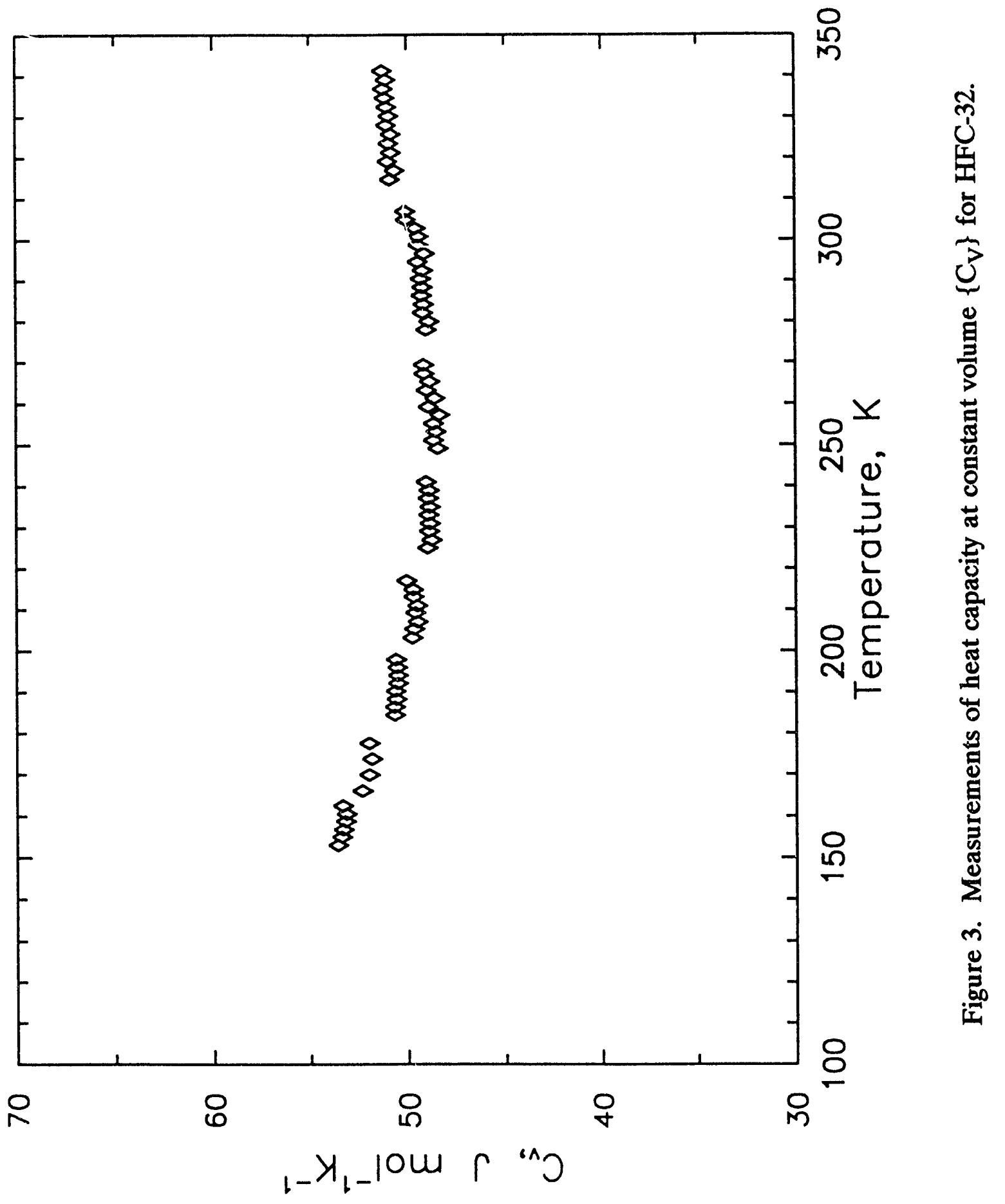




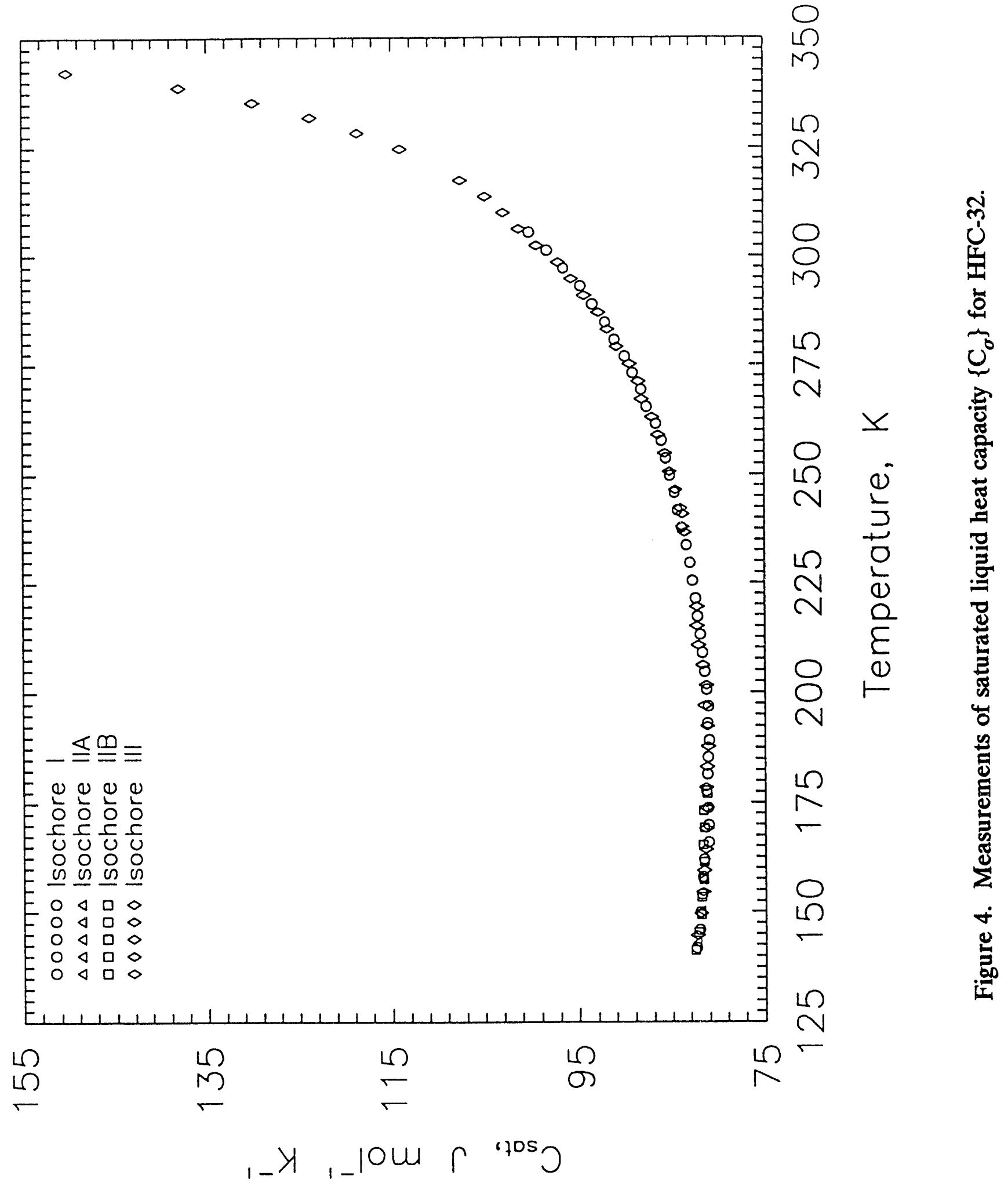




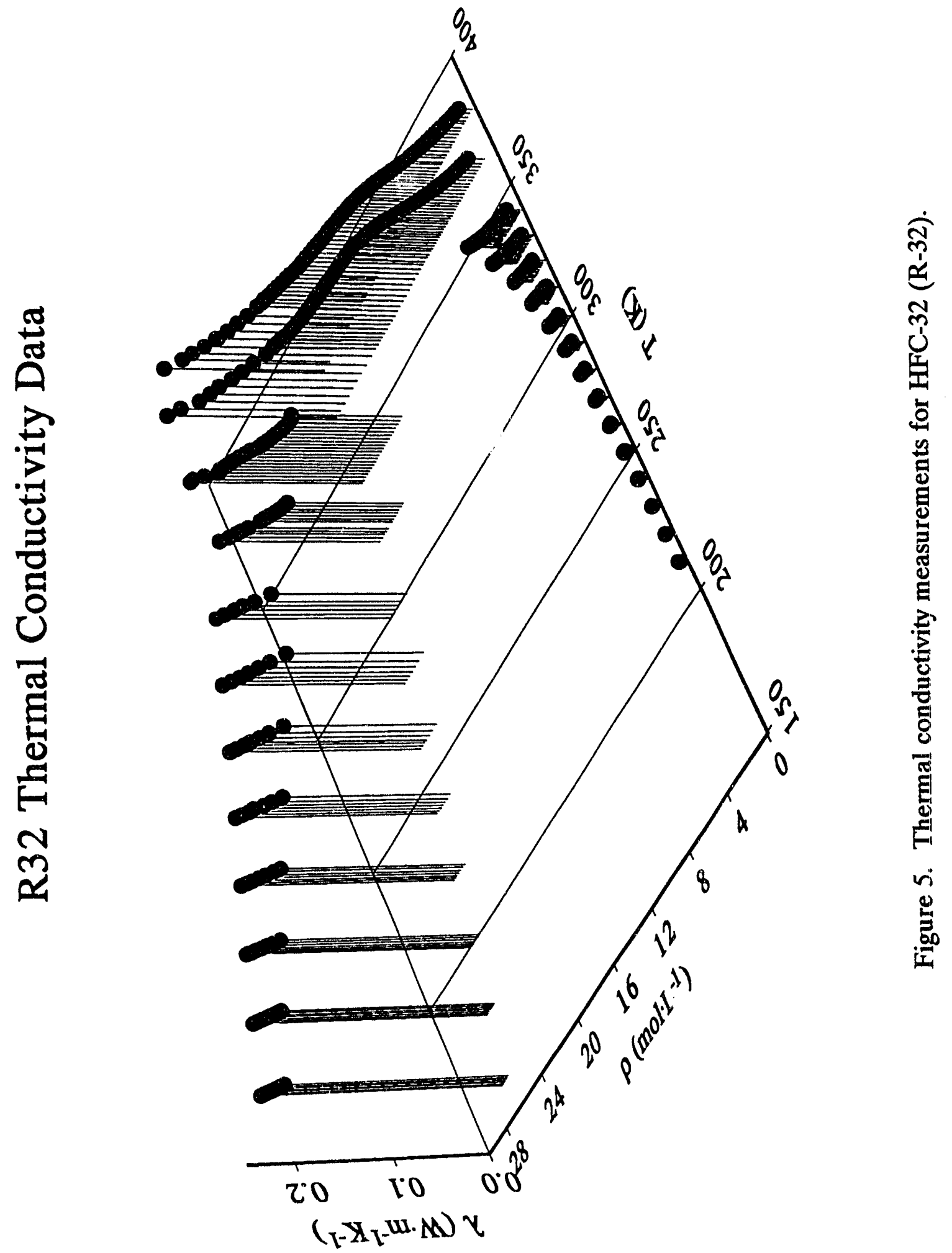




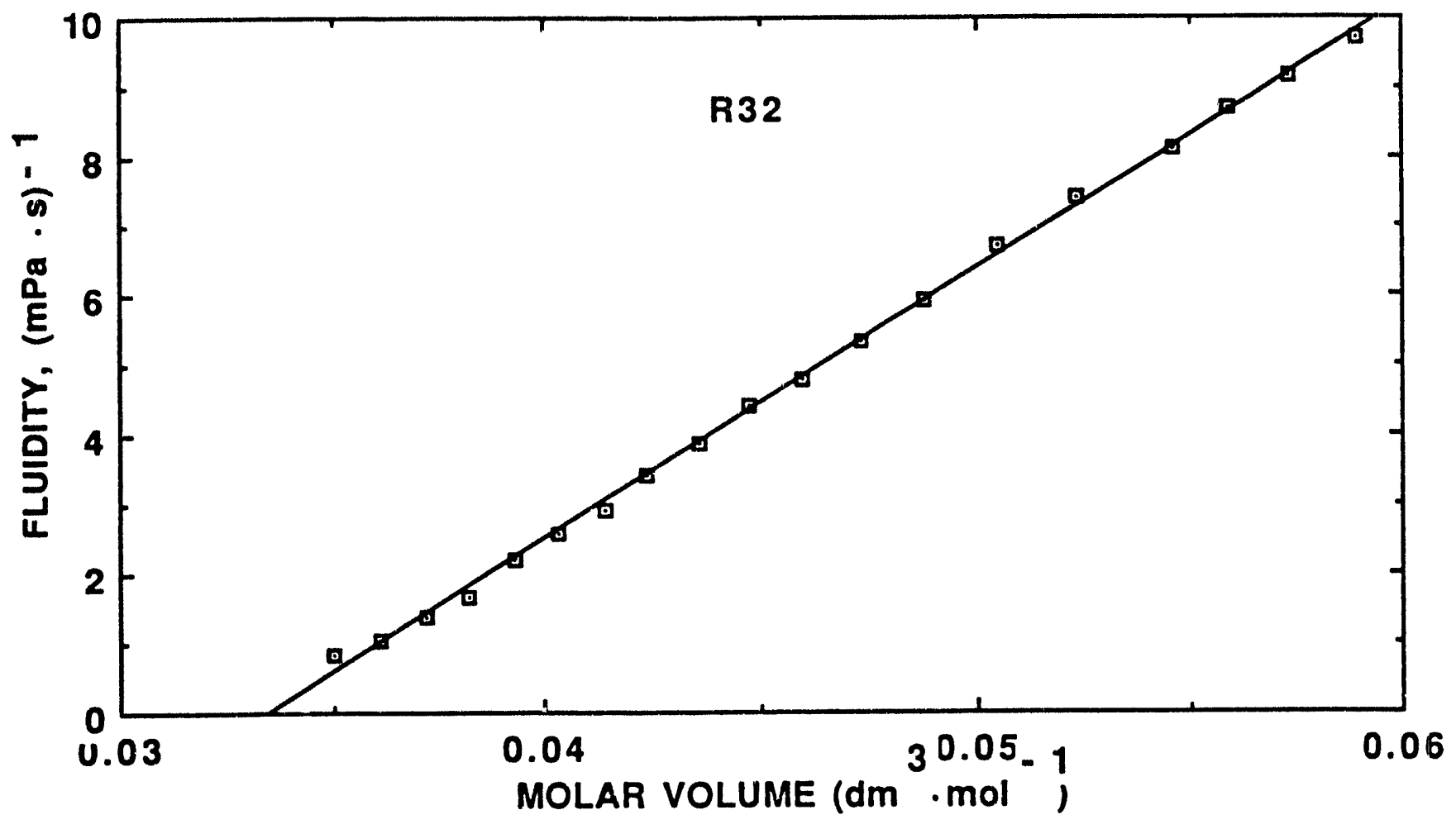

Figure 5. Dependence of HFC-32 (R-32) fluidity (reciprocal viscosity) data on molar volume. The data have been correlated with the equation, $1 / \eta=406.1(\mathrm{~V}-0.034)$, where the viscosity, $\eta$, is in $\mathrm{mPa} \cdot \mathrm{s}$ and the molar volume, $\mathrm{V}$, is in $\mathrm{dm}^{3} / \mathrm{mol}$. 


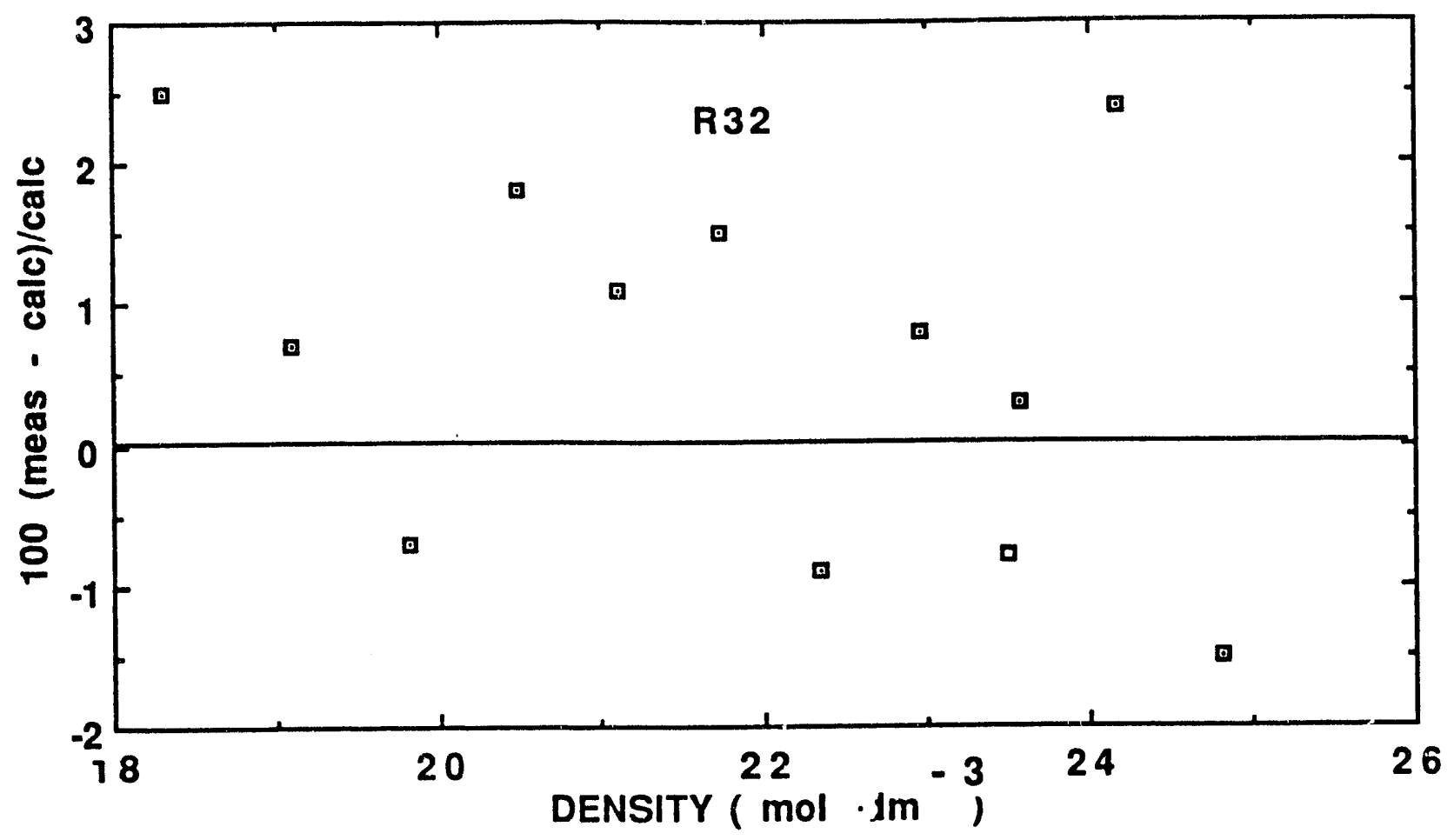

Figure 7. Comparison of HFC-32 (R-32) viscosity data with correlating equation. The differences are consistent with an imprecision of \pm 3 percent. 


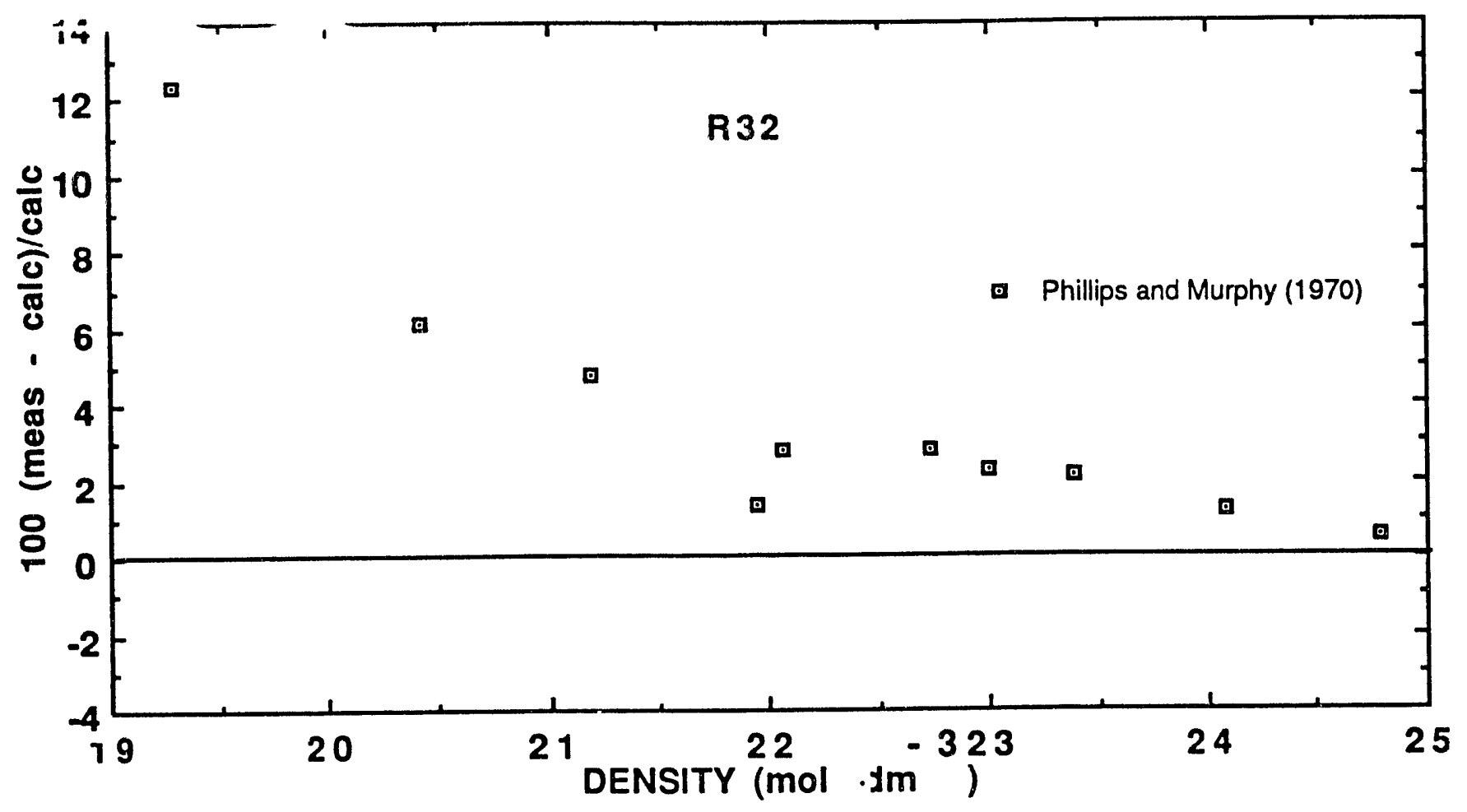

Figure 8. Comparison of Phillips and Murphy's HFC-32 (R-32) viscosity data with correlating equation. At high densities (low temperatures) the differences are within combined experimental errors. 


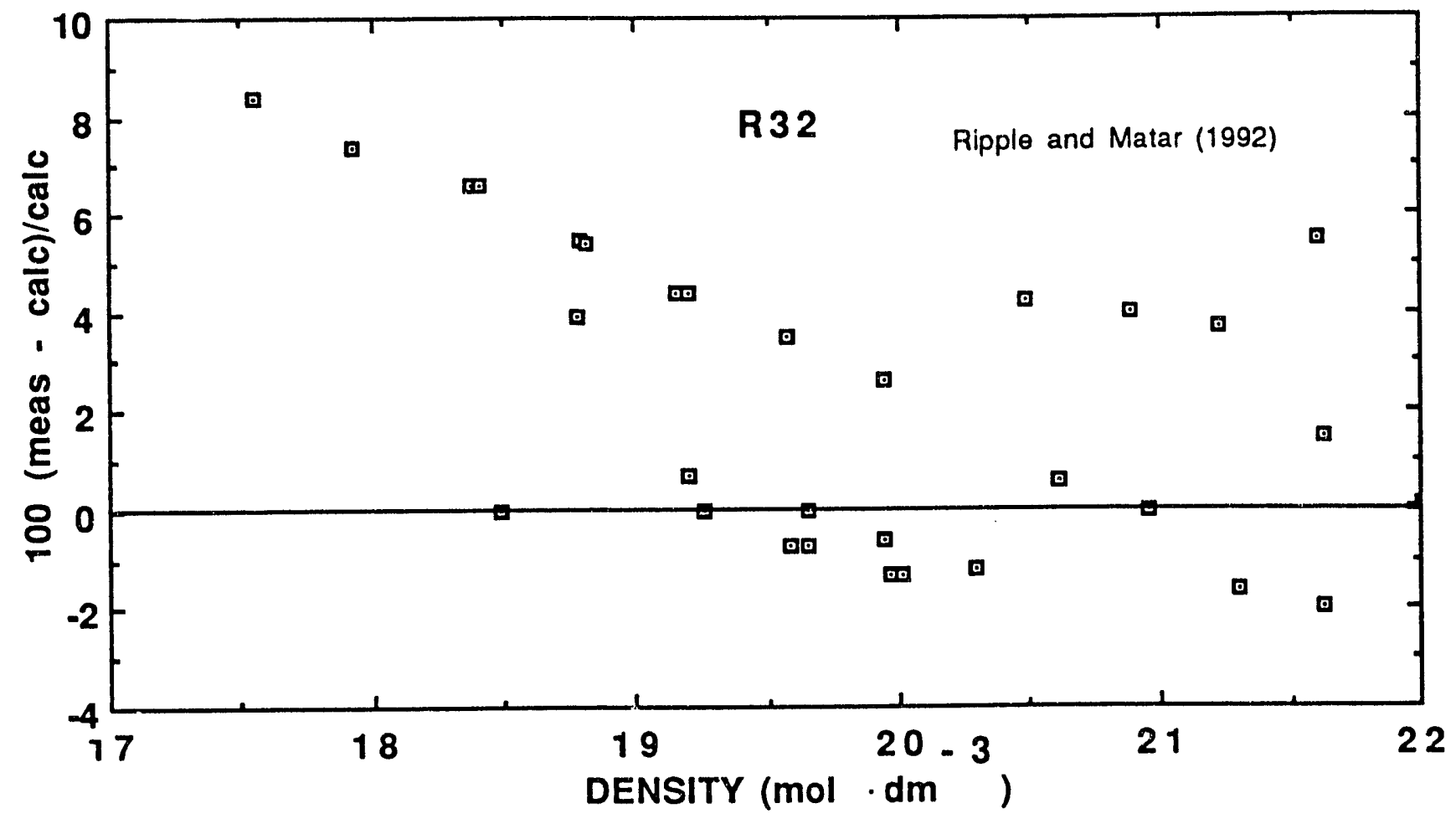

Figure 9. Comparison of Ripple and Matar's HFC-32 (R-32) viscosity data (NIST, Gaithersburg, MD, 1992) with correlating equation. At high densities (low temperatures), the differences are within combined experimental errors. 


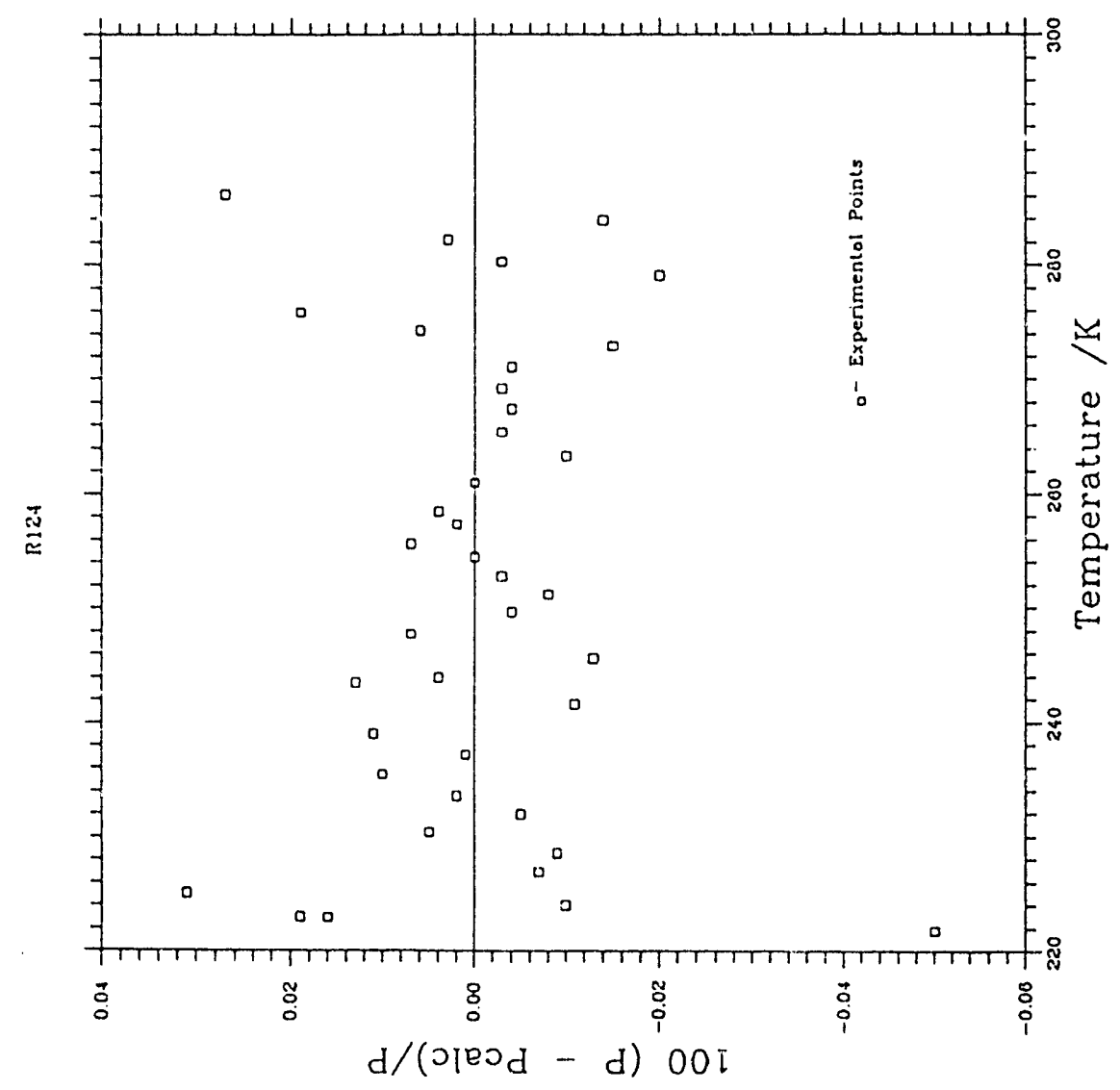

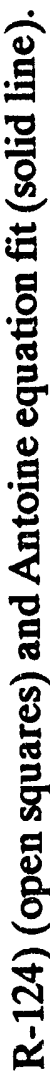

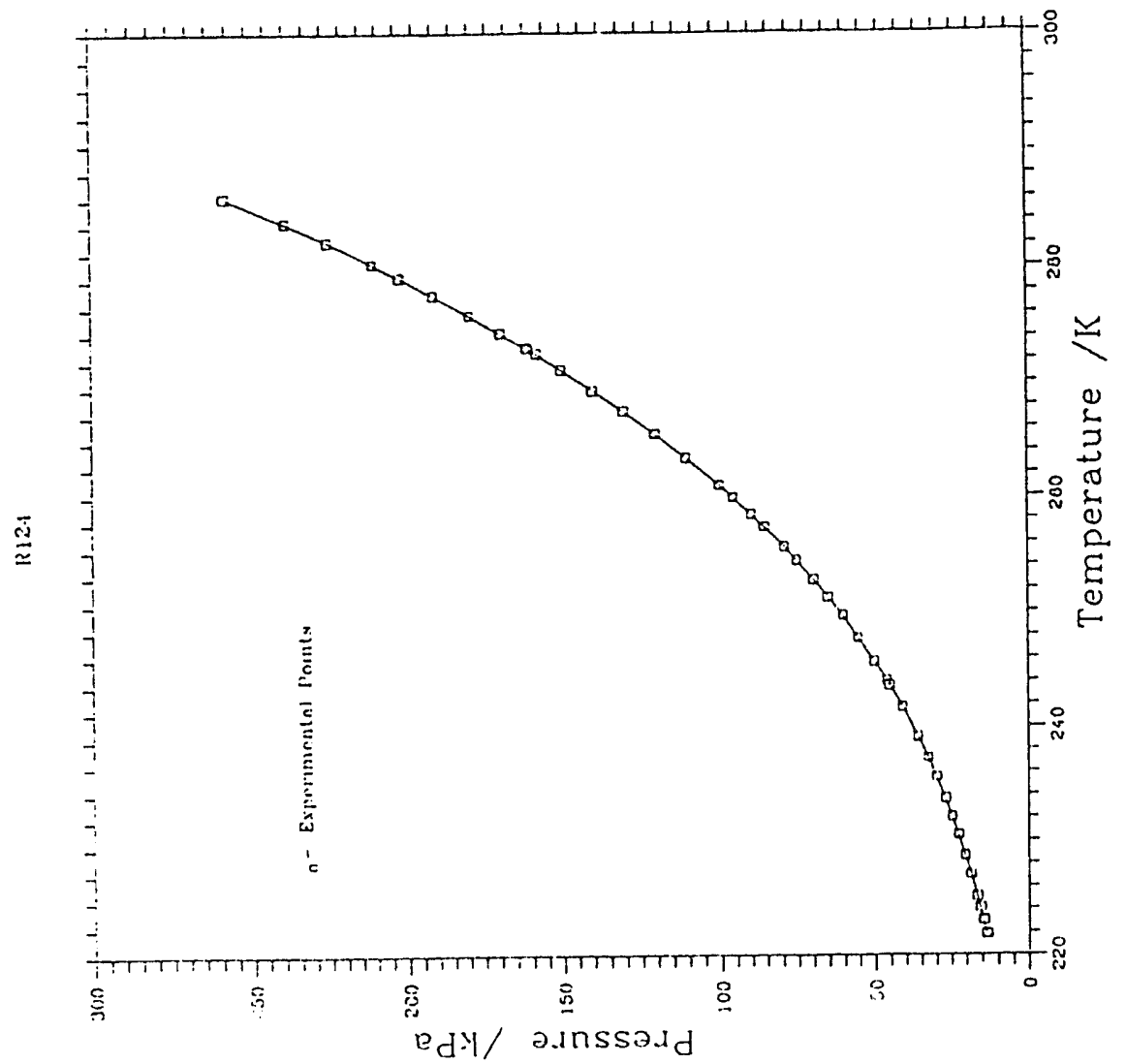




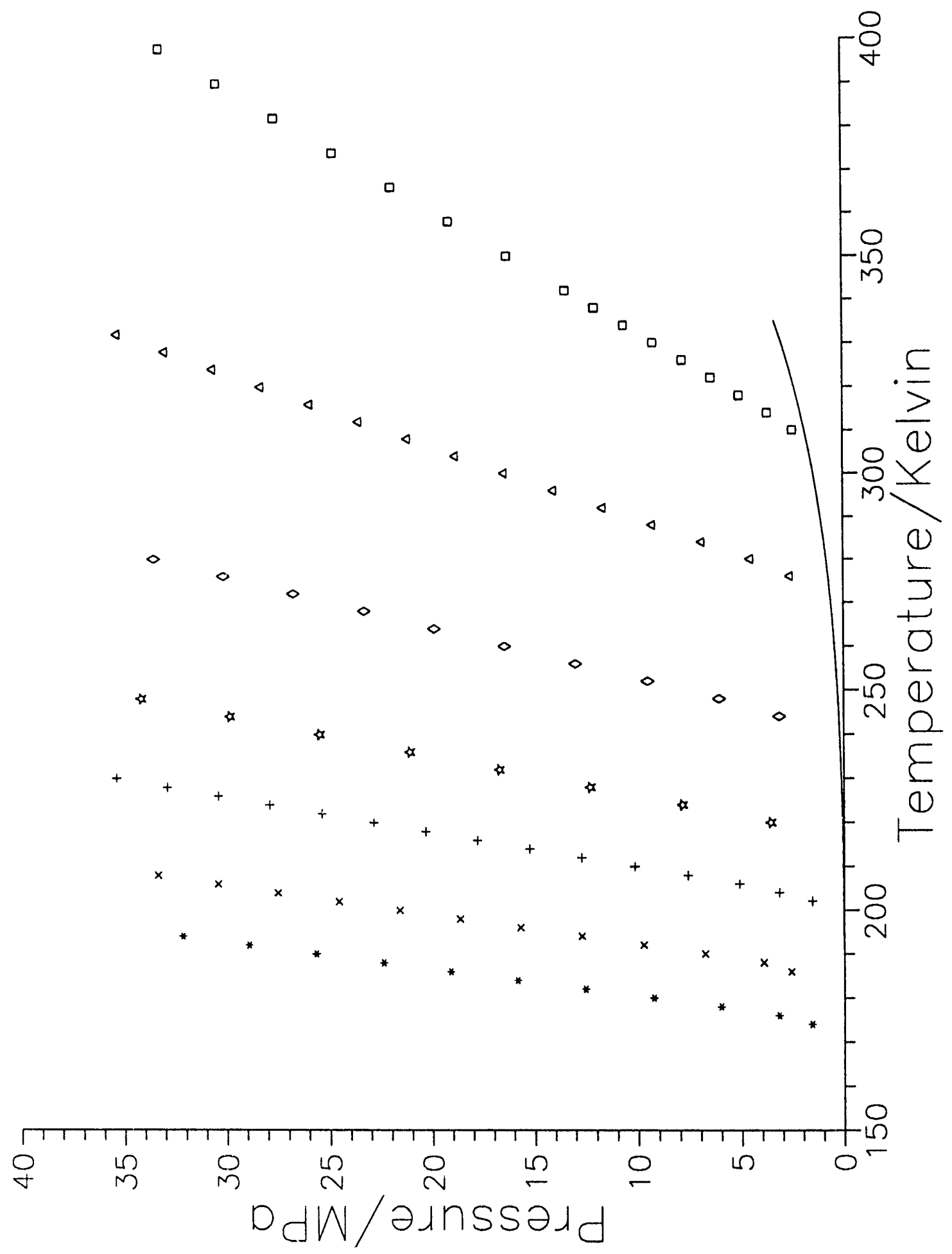

ปิ 

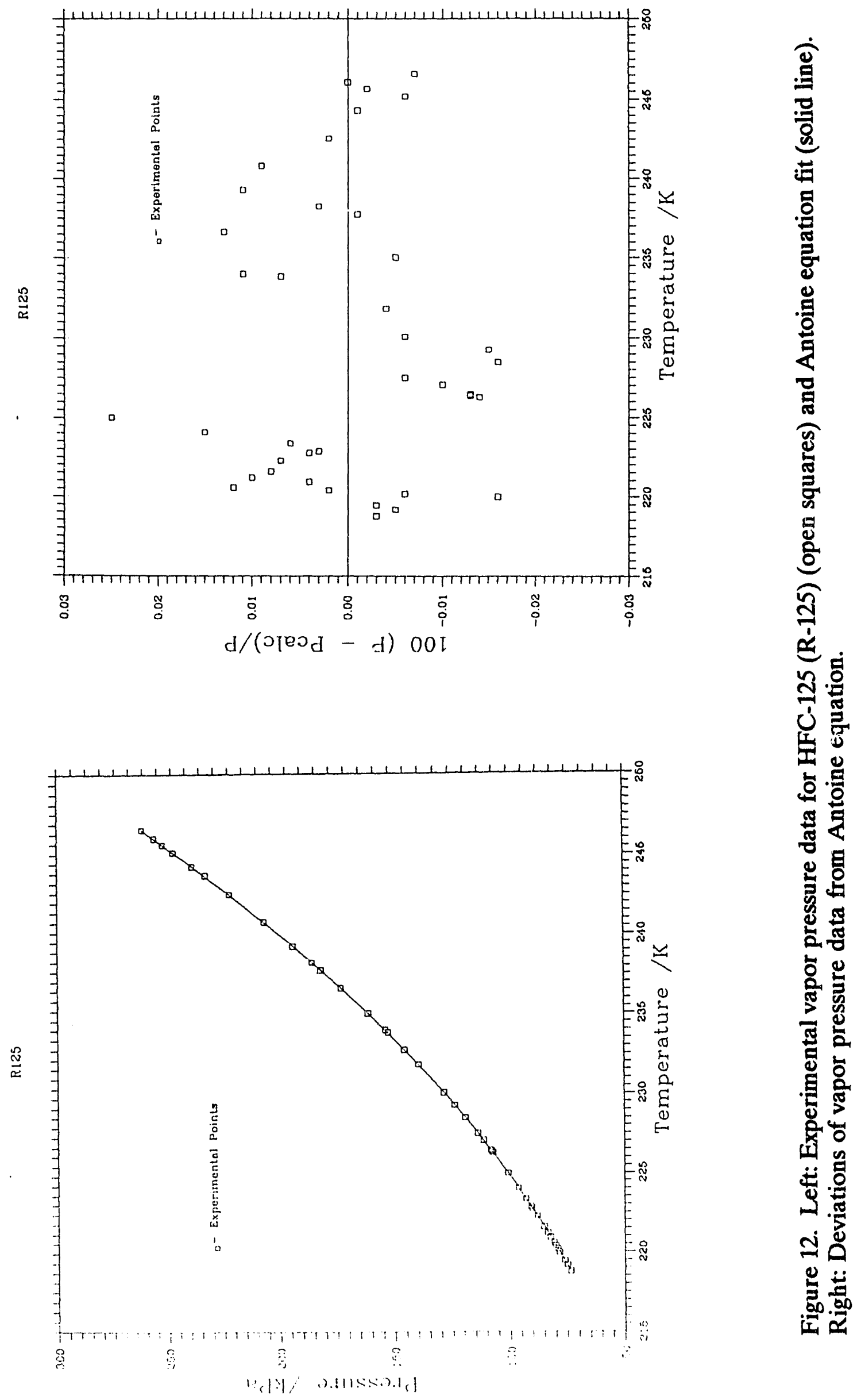


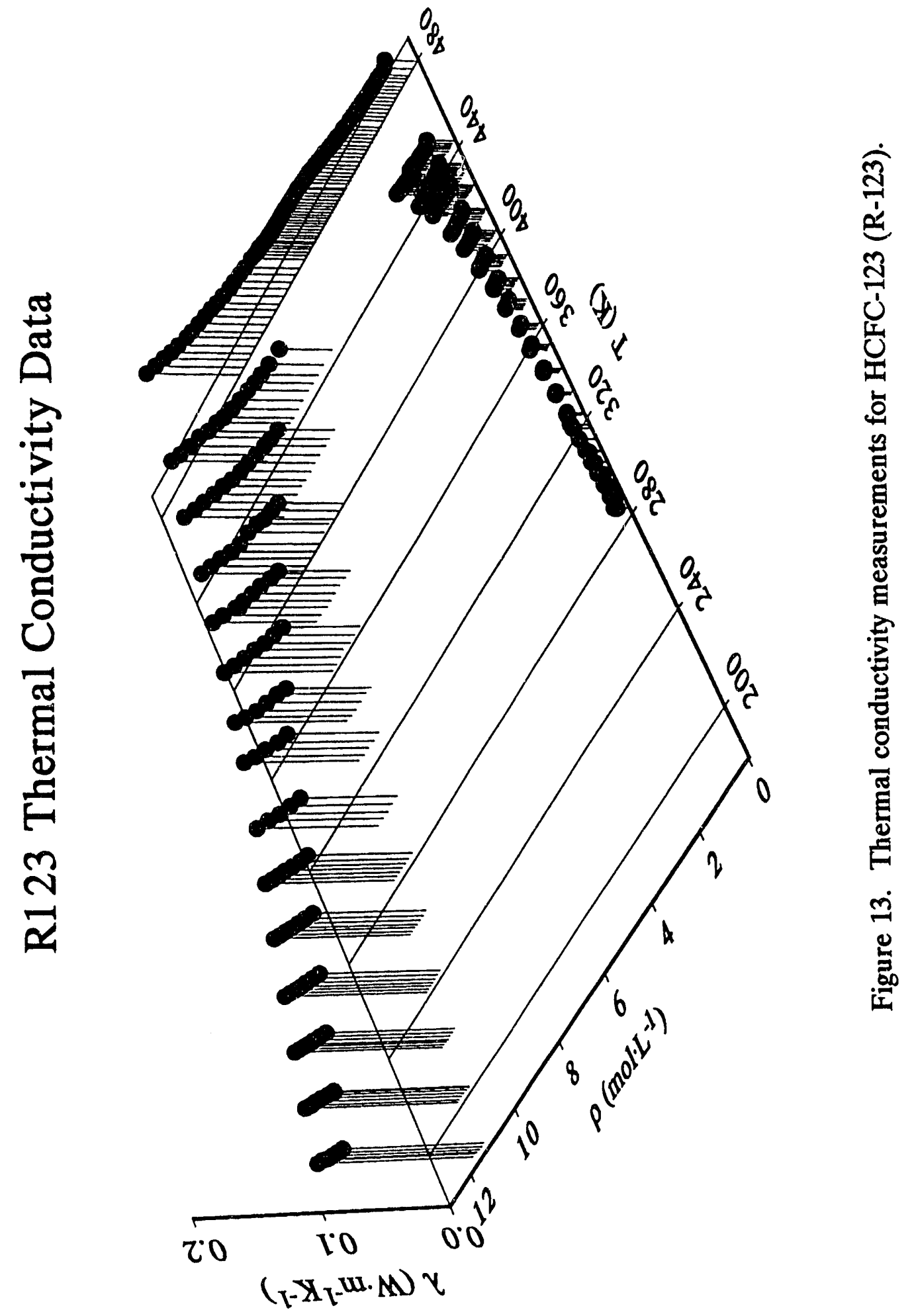


APPENDIX A

TABLES OF THERMOPHYSICAL PROPERTIES DATA

NOTE: SOME OF THESE DATA ARE PRELIMINARY AND SUBJECT TO CHANGE 
Table 1a. Vapor-phase PVT measurements for HFC-32 (SI units).

$\begin{array}{lll}\text { T/K } & \text { rho/g/cc } & \text { P/kPa } \\ 353.110 & .55188 & 6145.1 \\ 358.089 & .55176 & 7014.0 \\ 363.132 & .55164 & 7921.3 \\ 368.149 & .55153 & 8842.1 \\ 373.131 & .55141 & 9769.8 \\ 353.111 & .30979 & 5930.6 \\ 358.095 & .30973 & 6387.2 \\ 363.135 & .30966 & 6840.9 \\ 368.142 & .30960 & 7286.7 \\ 373.145 & .30953 & 7728.8 \\ 343.151 & .17391 & 4724.2 \\ 348.130 & .17388 & 4964.3 \\ 353.117 & .17384 & 5199.1 \\ 358.101 & .17380 & 5429.6 \\ 363.135 & .17377 & 5658.7 \\ 368.142 & .17373 & 5883.7 \\ 373.086 & .17369 & 6103.1 \\ 338.104 & .13323 & 4090.3 \\ 343.118 & .13320 & 4255.1 \\ 348.125 & .13318 & 4436.5 \\ 353.095 & .13315 & 4603.9 \\ 358.084 & .13312 & 4769.3 \\ 363.129 & .13309 & 4934.4 \\ 363.143 & .13309 & 4934.9 \\ 363.119 & .13309 & 4934.1 \\ 368.129 & .13306 & 5096.1 \\ 373.143 & .13304 & 5256.4 \\ 328.222 & .09765 & 3255.4 \\ 333.082 & .09763 & 3373.7 \\ 338.103 & .09761 & 3493.4 \\ 343.124 & .09759 & 3611.0 \\ 348.141 & .09757 & 3726.9 \\ 353.120 & .09755 & 3840.4 \\ 358.145 & .09753 & 3953.5 \\ 363.133 & .09751 & 4064.4 \\ 368.140 & .09749 & 4174.7 \\ 373.142 & .09747 & 4283.8 \\ 318.099 & .07486 & 2602.6 \\ 323.130 & .07484 & 2692.8 \\ 333.119 & .07481 & 2866.8 \\ 338.132 & .07479 & 2952.1 \\ 343.139 & .07478 & 3036.1 \\ 348.159 & .07476 & 3119.3 \\ 353.128 & .07475 & 3200.7 \\ 358.124 & .07473 & 3281.8 \\ 363.121 & .07472 & 3361.9 \\ 368.103 & .07470 & 3441.3 \\ 372.442 & .07469 & 3509.6 \\ 372.446 & .07469 & 3509.6 \\ & & \\ 353\end{array}$




$\begin{array}{llr}308.164 & .05484 & 1994.0 \\ 313.138 & .05483 & 2055.9 \\ 318.131 & .05482 & 2116.9 \\ 323.145 & .05481 & 2177.3 \\ 328.145 & .05480 & 2236.6 \\ 333.148 & .05479 & 2295.2 \\ 338.150 & .05477 & 2353.1 \\ 343.109 & .05476 & 2409.9 \\ 348.136 & .05475 & 2466.8 \\ 353.108 & .05474 & 2522.6 \\ 358.138 & .05473 & 2578.6 \\ 363.131 & .05472 & 2633.8 \\ 368.139 & .05470 & 2688.7 \\ 373.141 & .05469 & 2743.1 \\ 308.177 & .04202 & 1645.0 \\ 313.168 & .04201 & 1689.5 \\ 318.143 & .04201 & 1733.2 \\ 323.084 & .04200 & 1776.2 \\ 323.135 & .04200 & 1776.4 \\ 328.057 & .04199 & 1818.8 \\ 333.165 & .04198 & 1862.2 \\ 338.134 & .04197 & 1904.0 \\ 343.100 & .04196 & 1945.3 \\ 348.163 & .04195 & 1987.3 \\ 353.127 & .04194 & 2028.0 \\ 358.120 & .04194 & 2068.6 \\ 363.115 & .04193 & 2108.9 \\ 368.109 & .04192 & 2149.1 \\ 278.145 & .02361 & 882.3 \\ 281.187 & .02361 & 897.1 \\ 283.166 & .02361 & 907.1 \\ 288.132 & .02360 & 930.7 \\ 293.172 & .02360 & 954.2 \\ 298.125 & .02359 & 977.1 \\ 303.140 & .02359 & 1000.0 \\ 308.158 & .02358 & 1022.7 \\ 313.101 & .02358 & 1044.8 \\ 318.156 & .02357 & 1067.2 \\ 323.155 & .02357 & 1089.2 \\ 323.186 & .02357 & 1089.4 \\ 328.073 & .02356 & 1110.4 \\ 333.118 & .02356 & 1132.2 \\ 338.128 & .02355 & 1154.5 \\ 343.144 & .02355 & 1175.4 \\ 348.152 & .02354 & 1196.8 \\ 353.130 & .02354 & 1217.9 \\ 358.128 & .02353 & 1239.0 \\ 363.120 & .02353 & 1259.9 \\ 368.106 & .02352 & 1280.8 \\ 268.916 & .01325 & 512.1 \\ 268.834 & .01325 & 511.8 \\ 273.143 & .01325 & 522.6 \\ 278.129 & .01325 & 534.9 \\ 283.164 & .01325 & 547.2\end{array}$




$\begin{array}{lll}288.191 & .01324 & 559.4 \\ 293.167 & .01324 & 571.3 \\ 298.122 & .01324 & 583.1 \\ 303.138 & .01324 & 594.9 \\ 308.156 & .01323 & 606.7 \\ 313.161 & .01323 & 618.4 \\ 318.138 & .01323 & 630.0 \\ 322.930 & .01322 & 641.2 \\ 328.063 & .01322 & 653.1 \\ 333.121 & .01322 & 664.7 \\ 338.129 & .01322 & 676.1 \\ 343.140 & .01321 & 687.5 \\ 348.155 & .01321 & 698.9 \\ 353.133 & .01321 & 710.2 \\ 358.133 & .01321 & 721.4 \\ 363.170 & .01320 & 732.8 \\ 368.110 & .01320 & 743.8 \\ 268.133 & .00744 & 299.7 \\ 268.530 & .00744 & 300.2 \\ 273.158 & .00744 & 306.3 \\ 278.146 & .00744 & 312.8 \\ 283.161 & .00743 & 319.2 \\ 288.154 & .00743 & 325.5 \\ 293.144 & .00743 & 331.9 \\ 298.176 & .00743 & 338.3 \\ 303.133 & .00743 & 344.6 \\ 308.155 & .00743 & 350.9 \\ 313.112 & .00742 & 357.2 \\ 318.110 & .00742 & 363.4 \\ 323.155 & .00742 & 369.8 \\ 323.130 & .00742 & 369.9 \\ 323.175 & .00742 & 369.8 \\ 323.148 & .00742 & 369.8 \\ 323.150 & .00742 & 369.8 \\ 328.116 & .00742 & 376.1 \\ 333.070 & .00742 & 382.3 \\ 338.080 & .00742 & 388.5 \\ 343.157 & .00741 & 394.8 \\ 348.126 & .00741 & 400.9 \\ 353.146 & .00741 & 407.0 \\ 358.130 & .00741 & 413.1 \\ 363.120 & .00741 & 419.3 \\ 368.109 & .00741 & 425.4 \\ & & \end{array}$


Table 1b. Vaper-phase PVT measurements for HFC-32 (PI units).

\begin{tabular}{crr} 
T/F & rho/lb/ft & \multicolumn{1}{c}{ P/psia } \\
& & \\
175.928 & 34.452 & 891.27 \\
184.890 & 34.445 & 1017.30 \\
193.968 & 34.438 & 1148.89 \\
202.998 & 34.431 & 1282.43 \\
211.966 & 34.424 & 1416.99 \\
175.930 & 19.340 & 860.17 \\
184.901 & 19.336 & 926.39 \\
193.973 & 19.332 & 992.19 \\
202.986 & 19.327 & 1056.85 \\
211.991 & 19.323 & 1120.96 \\
158.002 & 10.857 & 685.19 \\
166.964 & 10.855 & 720.02 \\
175.941 & 10.853 & 754.07 \\
184.912 & 10.850 & 787.50 \\
193.973 & 10.848 & 820.72 \\
202.986 & 10.846 & 853.35 \\
211.885 & 10.843 & 885.18 \\
148.917 & 8.317 & 593.25 \\
157.942 & 8.316 & 618.61 \\
166.955 & 8.314 & 643.47 \\
175.901 & 8.312 & 667.74 \\
184.881 & 8.310 & 691.73 \\
193.962 & 8.309 & 715.68 \\
193.987 & 8.309 & 715.74 \\
193.944 & 8.309 & 715.63 \\
209.962 & 8.307 & 739.13 \\
211.987 & 8.305 & 762.38 \\
131.130 & 6.096 & 472.16 \\
139.878 & 6.095 & 489.31 \\
148.915 & 6.094 & 506.67 \\
157.953 & 6.092 & 523.74 \\
166.984 & 6.091 & 540.54 \\
175.946 & 6.090 & 557.00 \\
184.991 & 6.088 & 573.40 \\
193.969 & 6.087 & 589.49 \\
202.982 & 6.086 & 605.49 \\
211.986 & 6.085 & 621.31 \\
112.908 & 4.673 & 377.48 \\
121.964 & 4.672 & 390.56 \\
139.944 & 4.670 & 415.79 \\
148.968 & 4.669 & 428.16 \\
157.980 & 4.668 & 440.34 \\
167.016 & 4.667 & 452.41 \\
175.960 & 4.666 & 464.23 \\
184.953 & 4.665 & 475.98 \\
193.948 & 4.664 & 487.61 \\
202.915 & 4.663 & 499.12 \\
210.726 & 4.663 & 509.03 \\
210.733 & 4.663 & 509.02
\end{tabular}




$\begin{array}{rrr}95.025 & 3.424 & 289.21 \\ 103.978 & 3.423 & 298.18 \\ 112.966 & 3.422 & 307.04 \\ 121.991 & 3.422 & 31.5 .80 \\ 130.991 & 3.421 & 324.39 \\ 139.996 & 3.420 & 332.89 \\ 149.000 & 3.419 & 341.29 \\ 157.926 & 3.419 & 349.52 \\ 166.975 & 3.418 & 357.78 \\ 175.924 & 3.417 & 365.88 \\ 184.978 & 3.417 & 373.99 \\ 193.966 & 3.416 & 381.99 \\ 202.980 & 3.415 & 389.96 \\ 211.984 & 3.414 & 397.85 \\ 95.049 & 2.623 & 238.59 \\ 104.032 & 2.623 & 245.04 \\ 112.587 & 2.622 & 251.37 \\ 121.881 & 2.622 & 257.62 \\ 121.973 & 2.622 & 257.65 \\ 130.833 & 2.621 & 263.80 \\ 140.027 & 2.621 & 270.09 \\ 148.971 & 2.620 & 276.15 \\ 157.910 & 2.620 & 282.15 \\ 167.023 & 2.619 & 288.23 \\ 175.959 & 2.618 & 294.14 \\ 184.946 & 2.618 & 300.03 \\ 193.937 & 2.617 & 305.88 \\ 202.926 & 2.617 & 311.70 \\ 40.991 & 1.474 & 127.96 \\ 46.467 & 1.474 & 130.12 \\ 50.029 & 1.474 & 131.56 \\ 58.968 & 1.473 & 134.98 \\ 68.040 & 1.473 & 138.40 \\ 76.955 & 1.473 & 141.71 \\ 85.982 & 1.472 & 145.03 \\ 95.014 & 1.472 & 148.32 \\ 103.912 & 1.472 & 151.53 \\ 113.011 & 1.472 & 154.79 \\ 122.009 & 1.471 & 157.98 \\ 122.065 & 1.471 & 158.00 \\ 130.861 & 1.471 & 161.06 \\ 139.942 & 1.471 & 164.20 \\ 148.960 & 1.470 & 167.44 \\ 157.989 & 1.470 & 170.47 \\ 167.004 & 1.470 & 173.58 \\ 175.964 & 1.469 & 176.64 \\ 184.960 & 1.469 & 179.70 \\ 193.946 & 1.469 & 182.73 \\ 202.921 & 1.468 & 185.76 \\ 24.379 & .827 & 74.27 \\ 24.231 & .827 & 74.24 \\ 31.987 & .827 & 75.79 \\ 40.962 & .827 & 77.58 \\ 50.025 & .827 & 79.37\end{array}$




$\begin{array}{rrr}59.074 & .827 & 81.13 \\ 68.031 & .827 & 82.86 \\ 76.950 & .826 & 84.57 \\ 85.978 & .826 & 86.28 \\ 95.011 & .826 & 88.00 \\ 104.020 & .826 & 89.70 \\ 112.978 & .826 & 91.37 \\ 121.604 & .826 & 93.00 \\ 130.943 & .825 & 94.72 \\ 139.948 & .825 & 96.40 \\ 148.962 & .825 & 98.06 \\ 157.982 & .825 & 99.72 \\ 167.009 & .825 & 101.37 \\ 175.969 & .825 & 103.00 \\ 184.969 & .824 & 104.63 \\ 194.036 & .824 & 106.28 \\ 202.928 & .824 & 107.88 \\ 22.969 & .464 & 43.47 \\ 23.684 & .464 & 43.55 \\ 32.014 & .464 & 44.43 \\ 40.993 & .464 & 45.37 \\ 50.020 & .464 & 46.29 \\ 59.007 & .464 & 47.21 \\ 67.989 & .464 & 48.13 \\ 77.047 & .464 & 49.07 \\ 85.969 & .464 & 49.97 \\ 95.009 & .464 & 50.90 \\ 103.932 & .463 & 51.80 \\ 112.928 & .463 & 52.71 \\ 122.009 & .453 & 53.63 \\ 121.964 & .463 & 53.65 \\ 122.045 & .463 & 53.64 \\ 121.996 & .463 & 53.63 \\ 122.000 & .463 & 53.64 \\ 130.939 & .463 & 54.55 \\ 139.856 & .463 & 55.44 \\ 148.874 & .463 & 56.35 \\ 158.013 & .463 & 57.26 \\ 166.957 & .463 & 58.14 \\ 175.993 & .463 & 59.04 \\ 184.964 & .463 & 59.92 \\ 193.946 & .463 & 60.81 \\ 202.926 & .462 & 61.70 \\ & & \end{array}$


Table 2a. Compressed liquid density measurements for HFC-32 (SI units).

$\begin{array}{lcl}\text { T/R } & \text { rho/8/cc } & \text { P/kPa } \\ 242.593 & 1.1572 & 1999.3 \\ 242.596 & 1.1583 & 2499.3 \\ 242.583 & 1.1594 & 3001.3 \\ 242.584 & 1.1605 & 3501.1 \\ 242.583 & 1.1616 & 4001.1 \\ 242.588 & 1.1626 & 4502.0 \\ 242.586 & 1.1637 & 5002.4 \\ 242.594 & 1.1647 & 5502.3 \\ 242.588 & 1.1657 & 6002.1 \\ 242.592 & 1.1667 & 6503.0 \\ & & \\ 246.019 & 1.1470 & 2000.8 \\ 246.023 & 1.1481 & 2500.0 \\ 246.033 & 1.1492 & 3000.9 \\ 246.053 & 1.1503 & 3501.2 \\ 246.062 & 1.1514 & 4000.9 \\ 246.064 & 1.1525 & 4502.1 \\ 246.077 & 1.1535 & 5003.0 \\ 246.091 & 1.1546 & 5503.3 \\ 246.108 & 1.1556 & 6003.1 \\ 246.102 & 1.1567 & 6503.9 \\ & & \\ 250.555 & 1.1333 & 2000.6 \\ 250.570 & 1.1344 & 2500.8 \\ 250.568 & 1.1356 & 3000.9 \\ 250.578 & 1.1368 & 3501.2 \\ 250.581 & 1.1380 & 4001.2 \\ 250.581 & 1.1392 & 4502.0 \\ 250.572 & 1.1404 & 5002.9 \\ 250.582 & 1.1415 & 5503.3 \\ 250.587 & 1.1426 & 6003.0 \\ 250.572 & 1.1438 & 6503.9 \\ 255.116 & 1.1191 & 2000.5 \\ 255.120 & 1.1204 & 2500.8 \\ 255.125 & 1.1217 & 3001.1 \\ 255.130 & 1.1230 & 3501.3 \\ 255.135 & 1.1242 & 4000.9 \\ 255.136 & 1.1255 & 4501.9 \\ 255.141 & 1.1267 & 5-02.7 \\ 255.142 & 1.1280 & 5503.2 \\ 255.146 & 1.1292 & 6003.2 \\ 255.133 & 1.1304 & 6504.0 \\ 259.752 & 1.1045 & 2000.7 \\ 259.754 & 1.1059 & 2500.8 \\ 259.766 & 1.1072 & 3001.0 \\ 259.771 & 1.1086 & 3501.3 \\ 259.771 & 1.1100 & 4001.1\end{array}$




\begin{tabular}{|c|c|c|}
\hline $\begin{array}{l}259.779 \\
259.790 \\
259.799 \\
259.798 \\
259.806\end{array}$ & $\begin{array}{l}1.1113 \\
1.1126 \\
1.1139 \\
1.1152 \\
1.1165\end{array}$ & $\begin{array}{l}4502.3 \\
5002.9 \\
5503.2 \\
6003.0 \\
6503.9\end{array}$ \\
\hline $\begin{array}{l}264.488 \\
264.488 \\
264.501 \\
264.507 \\
264.529 \\
264.505 \\
264.520 \\
264.518 \\
264.511 \\
264.524\end{array}$ & $\begin{array}{l}1.0891 \\
1.0906 \\
1.0921 \\
1.0936 \\
1.0950 \\
1.0966 \\
1.0980 \\
1.0994 \\
1.1008 \\
1.1022\end{array}$ & $\begin{array}{l}2000.5 \\
2500.5 \\
3000.9 \\
3501.5 \\
4001.1 \\
4502.0 \\
5002.6 \\
5503.0 \\
6002.9 \\
6503.9\end{array}$ \\
\hline $\begin{array}{l}269.378 \\
269.382 \\
269.382 \\
269.388 \\
269.397 \\
269.398 \\
269.398 \\
269.401 \\
269.394 \\
269.397\end{array}$ & $\begin{array}{l}1.0728 \\
1.0745 \\
1.0761 \\
1.0778 \\
1.0793 \\
1.0809 \\
1.0825 \\
1.0840 \\
1.0856 \\
1.0871\end{array}$ & $\begin{array}{l}2000.4 \\
2500.8 \\
3000.9 \\
3501.1 \\
4001.2 \\
4502.2 \\
5003.0 \\
5503.1 \\
6003.1 \\
6503.9\end{array}$ \\
\hline $\begin{array}{l}274.000 \\
274.003 \\
274.003 \\
274.005 \\
274.009 \\
274.008 \\
274.010 \\
274.005 \\
274.009 \\
274.011\end{array}$ & $\begin{array}{l}1.0569 \\
1.0587 \\
1.0605 \\
1.0623 \\
1.0640 \\
1.0657 \\
1.0674 \\
1.0691 \\
1.0707 \\
1.0724\end{array}$ & $\begin{array}{l}2000.4 \\
2500.6 \\
3001.0 \\
3501.6 \\
4001.3 \\
4502.2 \\
5003.0 \\
5503.4 \\
6003.2 \\
6504.0\end{array}$ \\
\hline $\begin{array}{l}278.813 \\
278.813 \\
278.817 \\
278.828 \\
278.827 \\
278.834 \\
278.836 \\
278.828 \\
278.823 \\
278.816\end{array}$ & $\begin{array}{l}1.0398 \\
1.0419 \\
1.0438 \\
1.0457 \\
1.0476 \\
1.0495 \\
1.0513 \\
1.0532 \\
1.0550 \\
1.0568\end{array}$ & $\begin{array}{l}2000.3 \\
2500.7 \\
3001.0 \\
3501.7 \\
4001.4 \\
4502.2 \\
5002.9 \\
5503.2 \\
6003.0 \\
6504.0\end{array}$ \\
\hline $\begin{array}{l}283.647 \\
283.647 \\
283.647 \\
283.647\end{array}$ & $\begin{array}{l}1.0220 \\
1.0243 \\
1.0264 \\
1.0286\end{array}$ & $\begin{array}{l}2000.3 \\
2500.3 \\
3001.5 \\
3501.6\end{array}$ \\
\hline
\end{tabular}




$\begin{array}{lrl}283.647 & 1.0307 & 4001.3 \\ 283.643 & 1.0328 & 4502.3 \\ 283.642 & 1.0348 & 5003.0 \\ 283.643 & 1.0368 & 5503.4 \\ 283.643 & 1.0387 & 6003.3 \\ 283.643 & 1.0406 & 6504.1 \\ & & \\ 288.527 & 1.0033 & 2000.6 \\ 288.530 & 1.0057 & 2500.5 \\ 288.533 & 1.0081 & 3001.5 \\ 288.526 & 1.0105 & 3501.5 \\ 288.529 & 1.0128 & 4001.2 \\ 288.530 & 1.0151 & 4502.2 \\ 288.531 & 1.0173 & 5003.0 \\ 288.530 & 1.0195 & 5503.5 \\ 288.532 & 1.0217 & 6003.3 \\ 288.532 & 1.0238 & 6504.2 \\ & & \\ 293.459 & .9833 & 2000.5 \\ 293.460 & .9861 & 2500.4 \\ 293.460 & .9888 & 3001.5 \\ 293.459 & .9915 & 3501.5 \\ 293.465 & .9941 & 4001.3 \\ 293.463 & .9966 & 4502.2 \\ 293.462 & .9991 & 5003.1 \\ 293.458 & 1.0015 & 5503.4 \\ 293.461 & 1.0039 & 6003.3 \\ 293.461 & 1.0062 & 6504.3 \\ 298.407 & .9622 & 2000.5 \\ 298.410 & .9653 & 2500.4 \\ 298.419 & .9684 & 3001.4 \\ 298.415 & .9714 & 3501.4 \\ 298.417 & .9743 & 4001.4 \\ 298.419 & .9771 & 4502.3 \\ 298.426 & .9799 & 5003.2 \\ 298.425 & .9826 & 5503.4 \\ 298.427 & .9852 & 6003.2 \\ 303.425 & .9428 & 2501.4 \\ 303.428 & .9464 & 3001.4 \\ 303.425 & .9499 & 3501.6 \\ 303.423 & .9532 & 4001.5 \\ 303.421 & .9564 & 4502.4 \\ 303.420 & .9596 & 5003.2 \\ 303.418 & .9626 & 5503.5 \\ 303.423 & .9655 & 6003.3 \\ 303.347 & .9687 & 6504.2 \\ 308.304 & .9194 & 2501.2 \\ 308.288 & .9235 & 3001.3 \\ 308.254 & .9277 & 3501.6 \\ 308.253 & .9316 & 4001.5 \\ 308.255 & .9352 & 4502.4 \\ & & \end{array}$




$\begin{array}{lll}308.262 & .9388 & 5003.2 \\ 308.269 & .9422 & 5503.4 \\ 308.267 & .9455 & 6003.3 \\ 308.248 & .9488 & 6504.2 \\ & & \\ 313.123 & .8989 & 3001.8 \\ 313.118 & .9036 & 3501.7 \\ 313.127 & .9081 & 4001.4 \\ 313.131 & .9124 & 4502.4 \\ 313.134 & .9165 & 5003.1 \\ 313.144 & .9204 & 5503.5 \\ 313.156 & .9242 & 6003.3 \\ 313.148 & .9279 & 6504.2 \\ & & \\ 318.251 & .8756 & 3502.4 \\ 318.255 & .8811 & 4001.7 \\ 318.255 & .8863 & 4502.7 \\ 318.255 & .8912 & 5003.6 \\ 318.253 & .8959 & 5503.8 \\ 318.253 & .9004 & 6003.7 \\ 318.252 & .9046 & 6504.5 \\ 323.108 & .8457 & 3502.4 \\ 323.111 & .8526 & 4001.8 \\ 323.111 & .8590 & 4502.7 \\ 323.110 & .8650 & 5003.5 \\ 323.109 & .8706 & 5503.8 \\ 323.111 & .8758 & 6003.7 \\ 323.112 & .8808 & 6504.6 \\ 327.961 & .8162 & 3802.6 \\ 327.962 & .8197 & 4001.9 \\ 327.963 & .8281 & 4502.7 \\ 327.964 & .8356 & 5003.5 \\ 327.964 & .8426 & 5503.9 \\ 327.969 & .8490 & 6003.7 \\ 327.965 & .8550 & 6504.5 \\ & & \\ 332.789 & .7850 & 4202.4 \\ 332.796 & .7918 & 4502.8 \\ 332.800 & .8020 & 5003.5 \\ 332.800 & .8110 & 5503.8 \\ 332.800 & .8191 & 6003.6 \\ 332.801 & .8266 & 6504.5 \\ 337.789 & .7435 & 4503.0 \\ 337.792 & .7594 & 5003.4 \\ 337.793 & .7724 & 5503.7 \\ 337.791 & .7835 & 6003.6 \\ 337.793 & .7933 & 6504.5 \\ 347.638 & .7057 & 6504.4 \\ 347.702 & .6673 & 5803.9\end{array}$




$\begin{array}{lll}347.683 & .6801 & 6003.4 \\ 347.659 & .6912 & 6203.9 \\ 347.604 & .7009 & 6404.0 \\ 347.562 & .7057 & 6504.6 \\ & & \\ 348.531 & .6059 & 5303.7 \\ 348.538 & .6104 & 5353.7 \\ 348.568 & .6073 & 5403.5 \\ 348.569 & .6143 & 5453.4 \\ 348.592 & .6204 & 5503.3 \\ 348.608 & .6260 & 5553.7 \\ 348.602 & .6317 & 5603.6 \\ 348.599 & .6370 & 5653.5 \\ 348.601 & .6420 & 5703.4 \\ 348.605 & .6466 & 5753.2 \\ 348.622 & .6509 & 5803.8 \\ 348.630 & .6547 & 5853.7 \\ 348.567 & .6592 & 5903.5 \\ 348.579 & .6632 & 5953.2 \\ 348.568 & .6668 & 6003.0 \\ 348.569 & .6701 & 6053.6 \\ 348.578 & .6731 & 6103.5 \\ 348.584 & .6760 & 6153.4 \\ 348.602 & .6786 & 6203.4 \\ 348.612 & .6812 & 6253.5 \\ 343.609 & .6839 & 6304.1 \\ 348.608 & .6864 & 6354.0 \\ 348.596 & .6890 & 6403.9 \\ 348.605 & .6914 & 6454.0 \\ 348.595 & .6937 & 6504.0\end{array}$


Table 2b. Compressed 1iquid density measurements for HFC-32 (PI units).

\begin{tabular}{|c|c|c|}
\hline$T / F$ & $\mathrm{rho} / 1 \mathrm{~b} / \mathrm{ft} \mathrm{t}^{3}$ & P/psia \\
\hline $\begin{array}{l}-23.002 \\
-22.997 \\
-23.021 \\
-23.018 \\
-23.021 \\
-23.011 \\
-23.015 \\
-23.001 \\
-23.012 \\
-23.004\end{array}$ & $\begin{array}{l}72.242 \\
72.311 \\
72.382 \\
72.448 \\
72.515 \\
72.580 \\
72.646 \\
72.709 \\
72.774 \\
72.838\end{array}$ & $\begin{array}{l}289.97 \\
362.50 \\
435.31 \\
507.79 \\
580.30 \\
652.97 \\
725.53 \\
798.04 \\
870.53 \\
943.19\end{array}$ \\
\hline $\begin{array}{l}-16.836 \\
-16.829 \\
-16.810 \\
-16.774 \\
-16.759 \\
-16.754 \\
-16.730 \\
-16.706 \\
-16.675 \\
-16.686\end{array}$ & $\begin{array}{l}71.605 \\
71.675 \\
71.744 \\
71.810 \\
71.878 \\
71.947 \\
72.014 \\
72.079 \\
72.144 \\
72.211\end{array}$ & $\begin{array}{l}290.20 \\
362.59 \\
435.25 \\
507.81 \\
580.28 \\
652.98 \\
725.62 \\
798.18 \\
870.68 \\
943.31\end{array}$ \\
\hline $\begin{array}{l}-8.672 \\
-8.645 \\
-8.647 \\
-8.629 \\
-8.623 \\
-8.625 \\
-8.640 \\
-8.623 \\
-8.613 \\
-8.640\end{array}$ & $\begin{array}{l}70.747 \\
70.819 \\
70.896 \\
70.969 \\
71.043 \\
71.117 \\
71.191 \\
71.261 \\
71.332 \\
71.405\end{array}$ & $\begin{array}{l}290.17 \\
362.70 \\
435.24 \\
507.80 \\
580.33 \\
652.96 \\
725.61 \\
798.18 \\
870.67 \\
943.32\end{array}$ \\
\hline $\begin{array}{l}-.461 \\
-.454 \\
-.445 \\
-.436 \\
-.428 \\
-.425 \\
-.416 \\
-.414 \\
-.407 \\
-.431\end{array}$ & $\begin{array}{l}69.865 \\
69.946 \\
70.026 \\
70.106 \\
70.184 \\
70.263 \\
70.340 \\
70.417 \\
70.493 \\
70.570\end{array}$ & $\begin{array}{l}290.14 \\
362.71 \\
435.28 \\
507.81 \\
580.28 \\
652.95 \\
725.58 \\
798.17 \\
870.69 \\
943.32\end{array}$ \\
\hline $\begin{array}{l}7.884 \\
7.886 \\
7.909 \\
7.918 \\
7.917\end{array}$ & $\begin{array}{l}68.950 \\
69.037 \\
69.123 \\
69.208 \\
69.294\end{array}$ & $\begin{array}{l}290.18 \\
362.71 \\
435.26 \\
507.82 \\
580.31\end{array}$ \\
\hline
\end{tabular}




$\begin{array}{lll}7.933 & 69.377 & 653.00 \\ 7.952 & 69.459 & 725.61 \\ 7.969 & 69.541 & 798.17 \\ 7.966 & 69.623 & 870.66 \\ 7.981 & 69.702 & 943.32 \\ 16.408 & 67.992 & 290.15 \\ 16.408 & 68.087 & 362.67 \\ 16.433 & 68.179 & 435.24 \\ 16.443 & 68.272 & 507.85 \\ 16.482 & 68.361 & 580.31 \\ 16.439 & 68.456 & 652.96 \\ 16.467 & 68.544 & 725.56 \\ 16.463 & 68.633 & 798.14 \\ 16.450 & 68.722 & 870.65 \\ 16.473 & 68.807 & 943.31 \\ & & \\ 25.211 & 66.975 & 290.13 \\ 25.218 & 67.078 & 362.70 \\ 25.217 & 67.181 & 435.25 \\ 25.227 & 67.282 & 507.79 \\ 25.245 & 67.381 & 580.32 \\ 25.247 & 67.479 & 652.98 \\ 25.246 & 67.577 & 725.63 \\ 25.251 & 67.674 & 798.15 \\ 25.239 & 67.770 & 870.68 \\ 25.244 & 67.864 & 943.31 \\ & & \\ 33.529 & 65.977 & 290.13 \\ 33.536 & 66.091 & 362.69 \\ 33.536 & 66.204 & 435.26 \\ 33.538 & 66.314 & 507.87 \\ 33.546 & 66.423 & 580.34 \\ 33.544 & 66.530 & 652.99 \\ 33.549 & 66.636 & 725.63 \\ 33.539 & 66.741 & 798.20 \\ 33.545 & 66.844 & 870.69 \\ 33.550 & 66.945 & 943.33 \\ & & \\ 42.193 & 64.915 & 290.12 \\ 42.194 & 65.041 & 362.70 \\ 42.200 & 65.163 & 435.26 \\ 42.220 & 65.282 & 507.87 \\ 42.219 & 65.401 & 580.35 \\ 42.231 & 65.516 & 652.98 \\ 42.234 & 65.632 & 725.61 \\ 42.221 & 65.746 & 798.17 \\ 42.211 & 65.859 & 870.67 \\ 42.198 & 65.971 & 943.33 \\ 50.895 & 63.804 & 290.12 \\ 50.895 & 63.942 & 362.64 \\ 50.896 & 64.079 & 435.33 \\ 50.895 & 64.212 & 507.86\end{array}$




$\begin{array}{lll}50.895 & 64.343 & 580.34 \\ 50.887 & 64.473 & 653.00 \\ 50.886 & 64.599 & 725.62 \\ 50.888 & 64.723 & 798.19 \\ 50.887 & 64.844 & 870.70 \\ 50.888 & 64.964 & 943.35 \\ & & \\ 59.679 & 62.632 & 290.17 \\ 59.685 & 62.786 & 362.66 \\ 59.690 & 62.937 & 435.33 \\ 59.677 & 63.086 & 507.85 \\ 59.683 & 63.230 & 580.33 \\ 59.684 & 63.372 & 652.99 \\ 59.686 & 63.511 & 725.63 \\ 59.684 & 63.647 & 798.22 \\ 59.688 & 63.780 & 870.71 \\ 59.687 & 63.911 & 943.36 \\ & & \\ 68.556 & 61.388 & 290.14 \\ 68.558 & 61.561 & 362.65 \\ 68.558 & 61.731 & 435.33 \\ 68.557 & 61.897 & 507.85 \\ 68.567 & 62.058 & 580.34 \\ 68.564 & 62.216 & 653.00 \\ 68.562 & 62.371 & 725.63 \\ 68.555 & 62.522 & 798.21 \\ 68.560 & 62.669 & 870.71 \\ 68.561 & 62.814 & 943.36 \\ & & \\ 77.463 & 60.066 & 290.14 \\ 77.467 & 60.264 & 362.66 \\ 77.484 & 60.455 & 435.32 \\ 77.477 & 60.643 & 507.84 \\ 77.481 & 60.824 & 580.35 \\ 77.484 & 61.001 & 653.01 \\ 77.497 & 61.172 & 725.65 \\ 77.495 & 61.340 & 798.20 \\ 77.498 & 61.504 & 870.70 \\ 86.495 & 58.858 & 362.80 \\ 86.500 & 59.081 & 435.32 \\ 86.495 & 59.298 & 507.87 \\ 86.491 & 59.506 & 580.37 \\ 86.488 & 59.707 & 653.02 \\ 86.486 & 59.903 & 725.65 \\ 86.483 & 60.093 & 798.22 \\ 86.492 & 60.276 & 870.70 \\ 86.355 & 60.473 & 943.36 \\ & & \\ 95.277 & 57.393 & 362.77 \\ 95.248 & 57.655 & 435.31 \\ 95.187 & 57.912 & 507.86 \\ 95.185 & 58.155 & 580.37 \\ 95.189 & 58.385 & 653.02 \\ & & \end{array}$




$\begin{array}{lll}95.201 & 58.606 & 725.65 \\ 95.214 & 58.819 & 798.21 \\ 95.211 & 59.026 & 870.71 \\ 95.177 & 59.234 & 943.36 \\ 103.952 & 56.115 & 435.37 \\ 103.943 & 56.410 & 507.87 \\ 103.958 & 56.690 & 580.35 \\ 103.966 & 56.958 & 653.01 \\ 103.971 & 57.215 & 725.65 \\ 103.989 & 57.459 & 798.22 \\ 104.012 & 57.694 & 870.71 \\ 103.997 & 57.924 & 943.36 \\ & & \\ 113.183 & 54.664 & 507.97 \\ 113.190 & 55.006 & 580.40 \\ 113.190 & 55.330 & 653.06 \\ 113.189 & 55.637 & 725.71 \\ 113.186 & 55.929 & 798.26 \\ 113.186 & 56.208 & 870.77 \\ 113.183 & 56.474 & 943.40 \\ & & \\ 121.924 & 52.793 & 507.98 \\ 121.929 & 53.224 & 580.41 \\ 121.929 & 53.624 & 653.07 \\ 121.928 & 53.997 & 725.70 \\ 121.927 & 54.347 & 798.26 \\ 121.930 & 54.676 & 870.76 \\ 121.931 & 54.988 & 943.41 \\ 130.659 & 50.954 & 551.52 \\ 130.662 & 51.175 & 580.42 \\ 130.663 & 51.695 & 653.06 \\ 130.665 & 52.166 & 725.70 \\ 130.665 & 52.599 & 798.27 \\ 130.673 & 52.999 & 870.76 \\ 130.667 & 53.375 & 943.40 \\ & & \\ 139.351 & 49.006 & 609.50 \\ 139.362 & 49.429 & 653.07 \\ 139.371 & 50.065 & 725.69 \\ 139.369 & 50.628 & 798.26 \\ 139.370 & 51.135 & 870.75 \\ 139.371 & 51.600 & 943.40 \\ 148.350 & 46.414 & 653.11 \\ 148.356 & 47.407 & 725.68 \\ 148.358 & 48.219 & 798.25 \\ 148.354 & 48.912 & 870.75 \\ 148.357 & 49.522 & 943.39 \\ 166.079 & 44.053 & 943.39 \\ 166.145 & 40.729 & 812.84 \\ 166.193 & 41.658 & 841.78\end{array}$




$\begin{array}{lll}166.159 & 42.459 & 870.71 \\ 166.117 & 43.149 & 899.80 \\ 166.017 & 43.758 & 928.82 \\ 165.942 & 44.055 & 943.41 \\ 167.685 & 37.824 & 769.24 \\ 167.698 & 38.108 & 776.48 \\ 167.753 & 37.912 & 783.71 \\ 167.755 & 38.349 & 790.96 \\ 167.796 & 38.727 & 798.18 \\ 167.825 & 39.079 & 805.50 \\ 167.814 & 39.434 & 812.74 \\ 167.809 & 39.768 & 819.97 \\ 167.812 & 40.078 & 827.20 \\ 167.820 & 40.368 & 834.43 \\ 167.850 & 40.632 & 841.77 \\ 167.864 & 40.869 & 849.00 \\ 167.751 & 41.156 & 856.23 \\ 167.772 & 41.401 & 863.44 \\ 167.752 & 41.627 & 870.66 \\ 167.754 & 41.832 & 878.00 \\ 167.770 & 42.019 & 885.23 \\ 167.781 & 42.203 & 892.47 \\ 167.813 & 42.363 & 899.73 \\ 167.831 & 42.525 & 906.99 \\ 167.827 & 42.693 & 914.34 \\ 167.824 & 42.850 & 921.57 \\ 167.803 & 43.014 & 928.81 \\ 167.819 & 43.160 & 936.07 \\ 167.802 & 43.308 & 943.32\end{array}$


Table 3. Vapor pressures of HFC-32 obtained using the NIST Burnett apparatus.

$\begin{array}{llrl}\text { T/K } & \text { P/kPa } & \text { T/F } & \text { P/psia } \\ 268.154 & 690.56 & 23.007 & 10.015 \\ 273.163 & 813.62 & 32.023 & 11.800 \\ 278.137 & 951.22 & 40.976 & 13.796 \\ 283.184 & 1108.22 & 50.061 & 16.073 \\ 288.129 & 1280.21 & 58.962 & 18.567 \\ 293.121 & 1473.5 & 67.947 & 21.371 \\ 298.174 & 1690.81 & 77.043 & 24.523 \\ 303.122 & 1926.26 & 85.949 & 27.938 \\ 308.143 & 2189.5 & 94.987 & 31.756 \\ 313.122 & 2476.63 & 103.949 & 35.920 \\ 318.154 & 2794.65 & 113.007 & 40.532 \\ 318.169 & 2795.42 & 113.034 & 40.544 \\ 323.161 & 3140.65 & 122.019 & 45.551 \\ 328.202 & 3522.52 & 131.093 & 51.089 \\ 333.105 & 3927.61 & 139.919 & 56.965 \\ 338.150 & 4382.59 & 149.000 & 63.564 \\ 343.110 & 4871.98 & 157.928 & 70.662 \\ 348.081 & 5409.01 & 166.875 & 78.451\end{array}$


Table 4. Saturated vapor densities for HFC-32 (difluoromethane)

$\begin{array}{rrrrrr}\text { T/K } & \text { P/Bar } & \text { rho/mol/L } & \text { T/F } & \text { P/Psia } & \text { rho/lbs/ft3 } \\ 219 & 0.890 & 0.0509 & -65.469 & 12.921 & 0.1653 \\ 224 & 1.149 & 0.0647 & -56.469 & 16.669 & 0.2101 \\ 229 & 1.464 & 0.0813 & -47.469 & 21.234 & 0.2640 \\ 234 & 1.843 & 0.1010 & -38.469 & 26.737 & 0.3280 \\ 239 & 2.296 & 0.1244 & -29.469 & 33.303 & 0.4040 \\ 244 & 2.831 & 0.1519 & -20.469 & 41.067 & 0.4933 \\ 249 & 3.459 & 0.1840 & -11.469 & 50.170 & 0.5975 \\ 254 & 4.189 & 0.2214 & -2.469 & 60.760 & 0.7190 \\ 259 & 5.032 & 0.2645 & 6.530 & 72.993 & 0.8590 \\ 264 & 6.000 & 0.3142 & 15.530 & 87.029 & 1.0204 \\ 269 & 7.104 & 0.3713 & 24.530 & 103.037 & 1.2058 \\ 274 & 8.355 & 0.4367 & 33.530 & 121.190 & 1.4182 \\ 279 & 9.767 & 0.5114 & 42.530 & 141.668 & 1.6608 \\ 284 & 11.352 & 0.5967 & 51.530 & 164.646 & 1.9378 \\ 289 & 13.124 & 0.6941 & 60.530 & 190.347 & 2.2542 \\ 294 & 15.097 & 0.8054 & 69.530 & 218.963 & 2.6156 \\ 299 & 17.284 & 0.9326 & 78.530 & 250.683 & 3.0287 \\ 304 & 19.702 & 1.0785 & 87.530 & 285.753 & 3.5026 \\ 309 & 22.367 & 1.2466 & 96.530 & 324.405 & 4.0485 \\ 314 & 25.296 & 1.4414 & 105.530 & 366.887 & 4.6812 \\ 319 & 28.508 & 1.6690 & 114.530 & 413.473 & 5.4203 \\ 324 & 32.023 & 1.9385 & 123.530 & 464.454 & 6.2956 \\ 329 & 35.865 & 2.2631 & 132.530 & 520.177 & 7.3498 \\ 334 & 40.059 & 2.6649 & 141.530 & 581.006 & 8.6547 \\ 336 & 41.843 & 2.8552 & 145.130 & 606.881 & 9.2728 \\ 339 & 44.639 & 3.1841 & 150.530 & 647.434 & 10.3409 \\ 340 & 45.604 & 3.3084 & 152.330 & 661.430 & 10.7446 \\ 343 & 48.604 & 3.7403 & 157.730 & 704.941 & 12.1473\end{array}$


Table 5. Saturated liquid densities for HFC-32 (difluoromethane)

$\begin{array}{lrrrrr}\text { T/K } & \text { P/Bar } & \text { rho/mol/L } & \text { T/F } & \text { P/Psia } & \text { rho/1bs/ft3 } \\ 242.589 & 2.671 & 22.168 & -23.009 & 38.739 & 71.994 \\ 246.063 & 3.079 & 21.974 & -16.756 & 44.650 & 71.364 \\ 250.574 & 3.677 & 21.706 & -8.636 & 53.330 & 70.494 \\ 255.132 & 4.370 & 21.433 & -0.432 & 63.381 & 69.607 \\ 259.778 & 5.175 & 21.148 & 7.930 & 75.057 & 68.682 \\ 264.509 & 6.106 & 20.853 & 16.446 & 88.560 & 67.724 \\ 269.391 & 7.197 & 20.539 & 25.233 & 104.383 & 66.704 \\ 274.006 & 8.357 & 20.231 & 33.540 & 121.208 & 65.704 \\ 278.823 & 9.715 & 19.908 & 42.211 & 140.904 & 64.655 \\ 283.645 & 11.234 & 19.568 & 50.890 & 162.935 & 63.550 \\ 288.530 & 12.950 & 19.216 & 59.684 & 187.823 & 62.407 \\ 293.461 & 14.874 & 18.845 & 68.559 & 215.729 & 61.202 \\ 298.418 & 17.018 & 18.457 & 77.482 & 246.825 & 59.942 \\ 303.414 & 19.407 & 18.044 & 86.475 & 281.474 & 58.601 \\ 308.266 & 21.960 & 17.620 & 95.208 & 318.502 & 57.224 \\ 313.135 & 24.770 & 17.178 & 103.973 & 359.258 & 55.788 \\ 318.253 & 28.010 & 16.670 & 113.185 & 406.250 & 54.139 \\ 323.110 & 31.374 & 16.147 & 121.928 & 455.041 & 52.440 \\ 327.963 & 35.041 & 15.582 & 130.663 & 508.226 & 50.605 \\ 332.797 & 39.017 & 14.955 & 139.364 & 565.893 & 48.569 \\ 337.791 & 43.494 & 14.175 & 148.353 & 630.827 & 46.036\end{array}$


Table $6(a)$. Experimental 11quid heat capacity date for HFC-32.

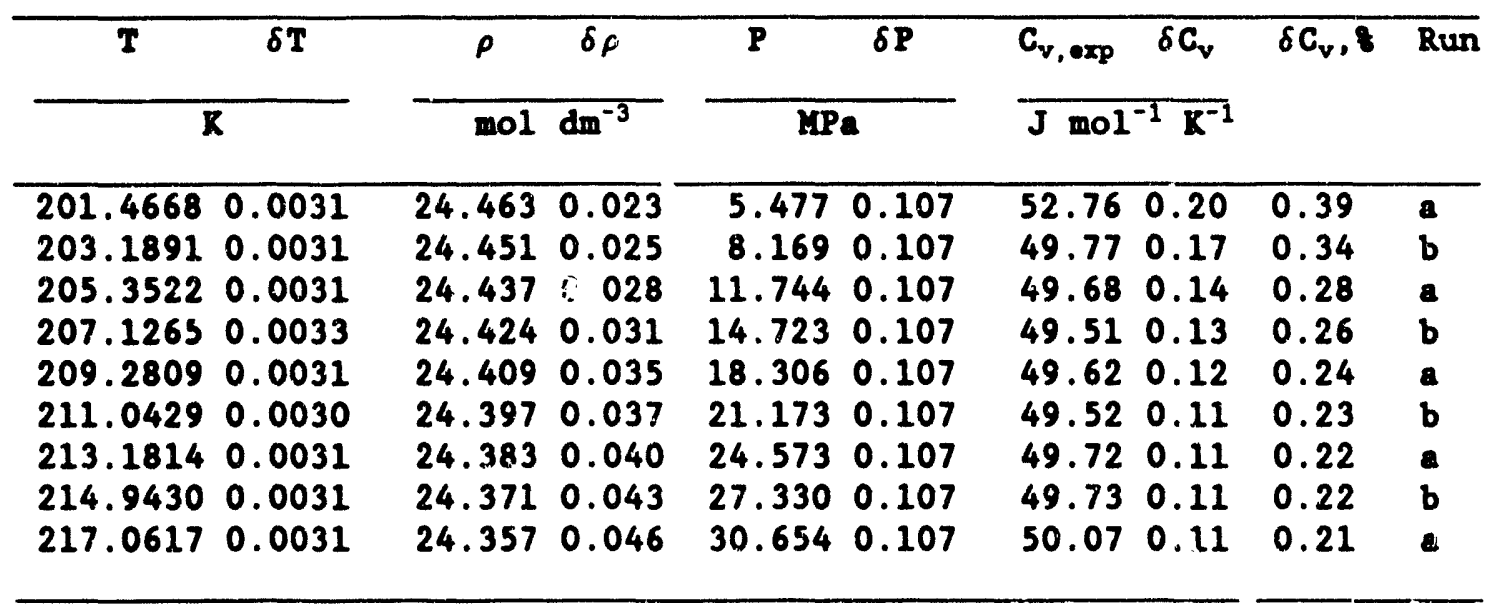

Table 6(b). Experimental 11quid heat capacity data for HFC-32.

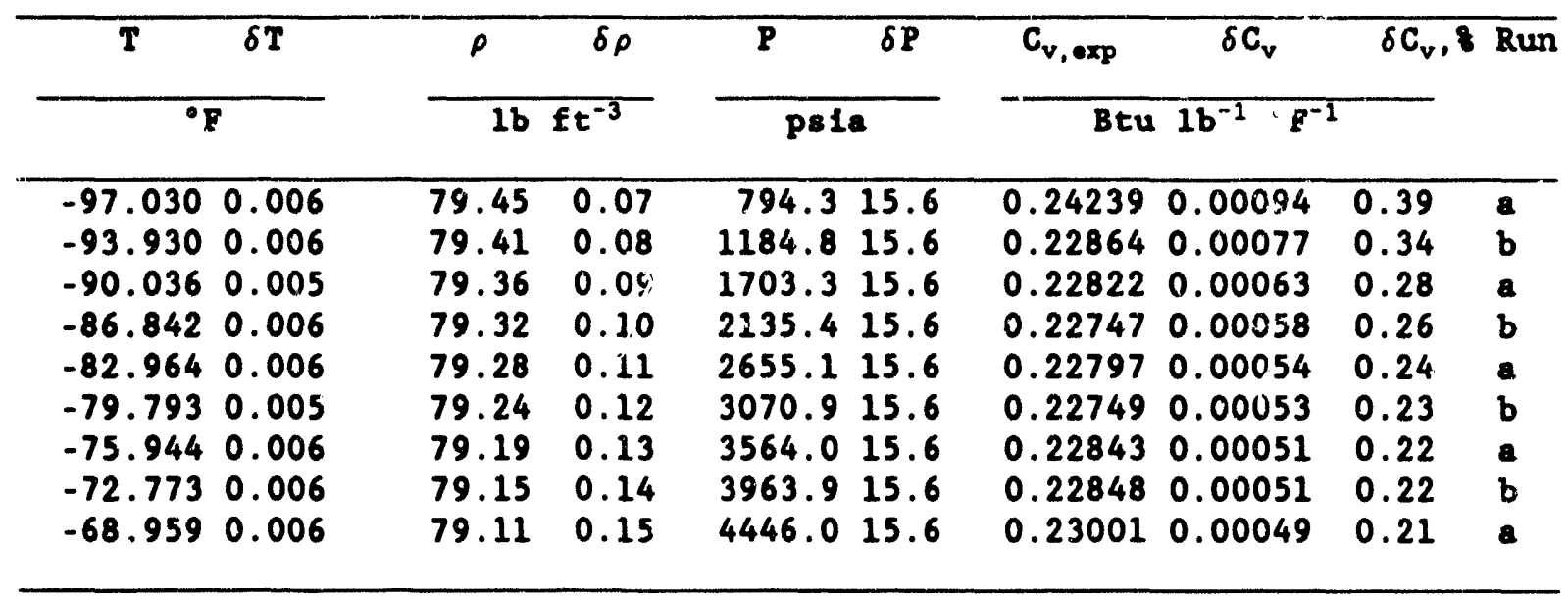


Table $7(a)$. Experimental liquid haat capacity data for HFC-32.

\begin{tabular}{|c|c|c|c|c|c|c|c|c|c|}
\hline $\bar{T}$ & $\delta T$ & $\rho$ & $\delta \rho$ & $\mathbf{P}$ & $\delta P$ & $C_{v, \exp }$ & & $\delta C_{v}, 8$ & $\overline{\text { Run }}$ \\
\hline \multicolumn{2}{|c|}{$\bar{R}$} & mol & $\mathrm{dm}^{-3}$ & \multicolumn{2}{|c|}{ MPa } & \multicolumn{4}{|c|}{$\mathrm{J} \mathrm{mol}{ }^{-1} \mathrm{~K}^{-1}$} \\
\hline $\begin{array}{l}223.0399 \\
225.1392 \\
226.9877 \\
229.1349 \\
231.0003 \\
233.1008 \\
234.9878 \\
237.0561 \\
238.95410 \\
240.9935\end{array}$ & $\begin{array}{l}0.0031 \\
0.0032 \\
0.0031 \\
0.0034 \\
0.0032 \\
0.0033 \\
0.0732 \\
0.0033 \\
0.0032 \\
0.0034\end{array}$ & $\begin{array}{l}23.372 \\
23.360 \\
23.349 \\
23.336 \\
23.325 \\
23.312 \\
23.301 \\
23.289 \\
23.278 \\
23.266\end{array}$ & $\begin{array}{l}0.021 \\
0.024 \\
0.026 \\
0.029 \\
0.031 \\
0.034 \\
0.036 \\
0.038 \\
0.041 \\
0.043\end{array}$ & $\begin{array}{r}5.067 \\
7.844 \\
10.421 \\
13.473 \\
16.121 \\
19.063 \\
21.658 \\
24.455 \\
26.999 \\
29.745\end{array}$ & $\begin{array}{l}0.059 \\
0.059 \\
0.059 \\
0.059 \\
0.059 \\
0.059 \\
0.059 \\
0.059 \\
0.059 \\
0.059\end{array}$ & $\begin{array}{l}52.53 \\
48.98 \\
48.78 \\
48.90 \\
48.87 \\
48.93 \\
48.88 \\
48.96 \\
48.92 \\
49.06\end{array}$ & $\begin{array}{l}0.20 \\
0.15 \\
0.13 \\
0.12 \\
0.11 \\
0.11 \\
0.10 \\
0.10 \\
0.10 \\
0.10\end{array}$ & $\begin{array}{l}0.39 \\
0.32 \\
0.27 \\
0.24 \\
0.22 \\
0.22 \\
0.21 \\
0.21 \\
0.20 \\
0.20\end{array}$ & $\begin{array}{l}a \\
b \\
a \\
b \\
a \\
b \\
a \\
b \\
a \\
b\end{array}$ \\
\hline
\end{tabular}

Table $7(b)$. Experimental 1iquid heat capacity data for HFC-32.

\begin{tabular}{|c|c|c|c|c|c|c|c|c|c|}
\hline$T$ & $\delta \mathrm{T}$ & $\rho$ & $\delta \rho$ & $\mathbf{P}$ & $\delta \mathbf{P}$ & $C_{v, \exp }$ & $\delta \mathrm{C}_{\mathrm{v}}$ & $\delta \mathrm{C}_{v}, 8$ & Run \\
\hline \multicolumn{2}{|c|}{${ }^{\circ} \mathrm{P}$} & \multicolumn{2}{|c|}{$\overline{1 b} z^{-3}$} & \multicolumn{2}{|c|}{ psia } & \multicolumn{3}{|c|}{ Btu $1 b^{-1} \cdot{ }^{0} \mathrm{~F}^{-1}$} & \\
\hline $\begin{array}{l}-58.198 \\
-54.419 \\
-51.092 \\
-47.227 \\
-43.869 \\
-40.088 \\
-36.692 \\
-32.969 \\
-29.553 \\
-25.882\end{array}$ & $\begin{array}{l}0.006 \\
0.006 \\
0.006 \\
0.006 \\
0.006 \\
0.006 \\
0.006 \\
0.006 \\
0.006 \\
0.006\end{array}$ & $\begin{array}{l}75.90 \\
75.87 \\
75.83 \\
75.79 \\
75.75 \\
75.71 \\
75.68 \\
75.64 \\
75.60 \\
75.56\end{array}$ & $\begin{array}{l}0.07 \\
0.08 \\
0.08 \\
0.09 \\
0.10 \\
0.11 \\
0.12 \\
0.12 \\
0.13 \\
0.14\end{array}$ & $\begin{array}{r}734.9 \\
1137.7 \\
1511.4 \\
1954.1 \\
2338.2 \\
2764.9 \\
3141.2 \\
3546.9 \\
3916.0 \\
4314.2\end{array}$ & $\begin{array}{l}8.5 \\
8.5 \\
8.5 \\
8.5 \\
8.5 \\
8.5 \\
8.5 \\
8.5 \\
8.5 \\
8.5\end{array}$ & $\begin{array}{l}0.24133 \\
0.22503 \\
0.22409 \\
0.22465 \\
0.22450 \\
0.22478 \\
0.22457 \\
0.22493 \\
0.22476 \\
0.22539\end{array}$ & $\begin{array}{l}0.00094 \\
0.00071 \\
0.00060 \\
0.00054 \\
0.00050 \\
0.00048 \\
0.00047 \\
0.00046 \\
0.00045 \\
0.00045\end{array}$ & $\begin{array}{l}0.39 \\
0.32 \\
0.27 \\
0.24 \\
0.22 \\
0.22 \\
0.21 \\
0.21 \\
0.20 \\
0.20\end{array}$ & $\begin{array}{l}a \\
b \\
a \\
b \\
a \\
b \\
a \\
b \\
a \\
b\end{array}$ \\
\hline
\end{tabular}


Table 8(a). Experimental liquid heat capacity data for HFC-32.

\begin{tabular}{|c|c|c|c|c|c|c|c|c|c|}
\hline $\mathbf{T}$ & $\delta \mathbf{T}$ & $\rho$ & $\delta \rho$ & $\mathbf{P}$ & $\delta P$ & $C_{v, \exp }$ & $\delta C_{v}$ & $\delta C_{v}, 8$ & Run \\
\hline \multicolumn{2}{|c|}{$\overline{\mathbf{K}}$} & mol & $\mathrm{dm}^{-3}$ & \multicolumn{2}{|c|}{$\mathbf{M P a}$} & \multicolumn{4}{|c|}{$\mathrm{J} \operatorname{mol}^{-1} \mathrm{~K}^{-1}$} \\
\hline $\begin{array}{l}247.0969 \\
249.1753 \\
251.1224 \\
253.2549 \\
255.1851 \\
257.3175 \\
259.2450 \\
261.3647 \\
263.2759 \\
265.3986 \\
267.3116 \\
269.4156\end{array}$ & $\begin{array}{l}0.0031 \\
0.0032 \\
0.0031 \\
0.0035 \\
0.0031 \\
0.0032 \\
0.0031 \\
0.0033 \\
0.0031 \\
0.0032 \\
0.0032 \\
0.0033\end{array}$ & $\begin{array}{l}22.066 \\
22.056 \\
22.047 \\
22.036 \\
22.026 \\
22.016 \\
22.006 \\
21.996 \\
21.987 \\
21.977 \\
21.968 \\
21.957\end{array}$ & $\begin{array}{l}0.019 \\
0.021 \\
0.023 \\
0.025 \\
0.027 \\
0.029 \\
0.031 \\
0.033 \\
0.035 \\
0.037 \\
0.038 \\
0.040\end{array}$ & $\begin{array}{r}3.950 \\
6.199 \\
8.420 \\
10.911 \\
13.175 \\
15.653 \\
17.859 \\
20.243 \\
22.361 \\
24.696 \\
26.807 \\
29.167\end{array}$ & $\begin{array}{l}0.054 \\
0.054 \\
0.054 \\
0.054 \\
0.054 \\
0.054 \\
0.054 \\
0.054 \\
0.054 \\
0.054 \\
0.054 \\
0.054\end{array}$ & $\begin{array}{l}52.43 \\
48.46 \\
48.69 \\
48.56 \\
48.69 \\
48.37 \\
48.94 \\
48.61 \\
49.04 \\
48.88 \\
49.17 \\
49.19\end{array}$ & $\begin{array}{l}0.21 \\
0.15 \\
0.12 \\
0.11 \\
0.10 \\
0.09 \\
0.09 \\
0.09 \\
0.09 \\
0.09 \\
0.09 \\
0.09\end{array}$ & $\begin{array}{l}0.40 \\
0.31 \\
0.25 \\
0.23 \\
0.21 \\
0.20 \\
0.19 \\
0.18 \\
0.18 \\
0.18 \\
0.18 \\
0.18\end{array}$ & $\begin{array}{l}a \\
b \\
a \\
b \\
a \\
b \\
a \\
b \\
a \\
b \\
a \\
b\end{array}$ \\
\hline
\end{tabular}

Table $8(b)$. Experimental liquid heat capacity data for HFC-32.

\begin{tabular}{|c|c|c|c|c|c|c|c|c|c|}
\hline $\mathbf{T}$ & $\delta T$ & $\rho$ & $\delta \rho$ & $\mathbf{P}$ & $\delta P$ & $C_{v, \exp }$ & $\delta G_{v}$ & $\delta C_{v}, 8$ & Run \\
\hline \multicolumn{2}{|c|}{${ }^{\circ} \mathrm{F}$} & \multicolumn{2}{|c|}{$1 b \mathrm{ft}^{-3}$} & \multicolumn{2}{|c|}{ psia } & \multicolumn{4}{|c|}{ Btu $1 b^{-1} \cdot{ }^{-1}$} \\
\hline $\begin{array}{r}-14.896 \\
-11.154 \\
-7.650 \\
-3.811 \\
-0.337 \\
3.502 \\
6.971 \\
10.786 \\
14.227 \\
18.047 \\
21.491 \\
25.278\end{array}$ & $\begin{array}{l}0.006 \\
0.006 \\
0.006 \\
0.006 \\
0.006 \\
0.006 \\
0.006 \\
0.006 \\
0.006 \\
0.006 \\
0.006 \\
0.006\end{array}$ & $\begin{array}{l}71.67 \\
71.63 \\
71.60 \\
71.57 \\
71.54 \\
71.50 \\
71.47 \\
71.44 \\
71.41 \\
71.38 \\
71.35 \\
71.31\end{array}$ & $\begin{array}{l}0.06 \\
0.07 \\
0.07 \\
0.08 \\
0.09 \\
0.09 \\
0.10 \\
0.11 \\
0.11 \\
0.12 \\
0.12 \\
0.13\end{array}$ & $\begin{array}{r}572.9 \\
899.1 \\
1221.3 \\
1582.5 \\
1910.8 \\
2270.3 \\
2590.2 \\
2936.0 \\
3243.2 \\
3581.9 \\
3888.0 \\
4230.3\end{array}$ & $\begin{array}{l}7.8 \\
7.8 \\
7.8 \\
7.8 \\
7.8 \\
7.8 \\
7.8 \\
7.8 \\
7.8 \\
7.8 \\
7.8 \\
7.8\end{array}$ & $\begin{array}{l}0.24089 \\
0.22264 \\
0.22370 \\
0.22309 \\
0.22370 \\
0.22220 \\
0.22483 \\
0.22333 \\
0.22530 \\
0.22457 \\
0.22589 \\
0.22600\end{array}$ & $\begin{array}{l}0.00096 \\
0.00070 \\
0.00057 \\
0.00050 \\
0.00046 \\
0.00044 \\
0.00042 \\
0.00041 \\
0.00040 \\
0.00040 \\
0.00040 \\
0.00040\end{array}$ & $\begin{array}{l}0.40 \\
0.31 \\
0.25 \\
0.23 \\
0.21 \\
0.20 \\
0.19 \\
0.18 \\
0.18 \\
0.18 \\
0.18 \\
0.18\end{array}$ & $\begin{array}{l}a \\
b \\
a \\
b \\
a \\
b \\
a \\
b \\
a \\
b \\
a \\
b\end{array}$ \\
\hline
\end{tabular}


Table $9(a)$. Experimental liquid heat capacity data for HFC-32.

\begin{tabular}{|c|c|c|c|c|c|c|c|c|c|}
\hline $\mathbf{T}$ & $\delta \mathbf{T}$ & $\rho$ & $\delta \rho$ & $\mathbf{P}$ & $\delta P$ & $C_{v, \exp }$ & $\delta C_{v}$ & $\delta C_{v}, 8$ & $\overline{\text { Run }}$ \\
\hline \multicolumn{2}{|c|}{$\overline{\mathbf{K}}$} & \multicolumn{2}{|c|}{ mol $\mathrm{dm}^{-3}$} & \multicolumn{2}{|c|}{$\overline{\mathbf{M P a}}$} & \multicolumn{4}{|c|}{$\mathrm{J} \mathrm{mol}^{-1} \mathrm{~K}^{-1}$} \\
\hline $\begin{array}{l}275.8475 \\
277.9247 \\
279.9747 \\
282.0959 \\
284.1547 \\
286.2690 \\
288.3385 \\
290.4273 \\
292.5119 \\
294.5814 \\
296.6696 \\
298.7243 \\
300.8062 \\
302.8544 \\
304.9452 \\
306.9722\end{array}$ & $\begin{array}{l}0.0034 \\
0.0033 \\
0.0035 \\
0.0033 \\
0.0033 \\
0.0033 \\
0.0032 \\
0.0032 \\
0.0031 \\
0.0032 \\
0.0033 \\
0.0034 \\
0.0032 \\
0.0032 \\
0.0035 \\
0.0034\end{array}$ & $\begin{array}{l}20.321 \\
20.314 \\
20.306 \\
20.298 \\
20.290 \\
20.282 \\
20.274 \\
20.266 \\
20.258 \\
20.250 \\
20.242 \\
20.235 \\
20.227 \\
20.219 \\
20.211 \\
20.203\end{array}$ & $\begin{array}{l}0.018 \\
0.019 \\
0.021 \\
0.022 \\
0.023 \\
0.025 \\
0.026 \\
0.028 \\
0.029 \\
0.030 \\
0.032 \\
0.033 \\
0.034 \\
0.036 \\
0.037 \\
0.038\end{array}$ & $\begin{array}{r}4.042 \\
5.795 \\
7.567 \\
9.429 \\
11.251 \\
13.126 \\
14.959 \\
16.801 \\
18.627 \\
20.428 \\
22.232 \\
23.996 \\
25.776 \\
27.524 \\
29.309 \\
31.047\end{array}$ & $\begin{array}{l}0.040 \\
0.040 \\
0.040 \\
0.040 \\
0.040 \\
0.040 \\
0.040 \\
0.040 \\
0.040 \\
0.040 \\
0.040 \\
0.040 \\
0.040 \\
0.040 \\
0.040 \\
0.040\end{array}$ & $\begin{array}{l}52.42 \\
49.05 \\
48.91 \\
49.22 \\
49.19 \\
49.27 \\
49.24 \\
49.31 \\
49.22 \\
49.47 \\
49.12 \\
49.44 \\
49.43 \\
49.58 \\
50.07 \\
50.10\end{array}$ & $\begin{array}{l}0.21 \\
0.15 \\
0.12 \\
0.10 \\
0.09 \\
0.09 \\
0.08 \\
0.08 \\
0.08 \\
0.08 \\
0.07 \\
0.08 \\
0.07 \\
0.07 \\
0.08 \\
0.07\end{array}$ & $\begin{array}{l}0.39 \\
0.30 \\
0.24 \\
0.20 \\
0.18 \\
0.17 \\
0.16 \\
0.16 \\
0.15 \\
0.15 \\
0.15 \\
0.15 \\
0.15 \\
0.15 \\
0.15 \\
0.15\end{array}$ & $\begin{array}{l}a \\
b \\
a \\
b \\
a \\
b \\
a \\
b \\
a \\
b \\
a \\
b \\
a \\
b \\
a \\
b\end{array}$ \\
\hline
\end{tabular}

Table $9(b)$. Experimental liquid heat capacity data for HFC-32.

\begin{tabular}{|c|c|c|c|c|c|c|c|c|c|}
\hline $\mathbf{T}$ & $\delta T$ & $\rho$ & $\delta \rho$ & $\mathbf{P}$ & $\overline{\delta P}$ & $\overline{C_{v, \text { exp }}}$ & $\delta \mathrm{C}_{\mathrm{v}}$ & $\delta C_{v}, 8$ & Run \\
\hline \multicolumn{2}{|c|}{${ }^{\circ} \mathbf{F}$} & \multicolumn{2}{|c|}{$1 \mathrm{~b} \mathrm{ft}^{-3}$} & \multicolumn{2}{|c|}{ psia } & \multicolumn{3}{|c|}{ Btu $1 b^{-1}{ }^{\circ} \mathbf{F}^{-1}$} & \\
\hline 36.855 & 0.006 & 66.00 & 0.06 & 586.3 & 5.8 & 0.24084 & 0.00094 & 0.39 & a \\
\hline 40.594 & 0.006 & 65.97 & 0.06 & 840.5 & 5.8 & 0.22532 & 0.00067 & 0.30 & b \\
\hline 44.285 & 0.006 & 65.95 & 0.07 & 1097.5 & 5.8 & 0.22468 & 0.00053 & 0.24 & a \\
\hline 48.103 & 0.006 & 65.92 & 0.07 & 1367.6 & 5.8 & 0.22611 & 0.00046 & 0.20 & $\mathbf{b}$ \\
\hline 51.808 & 0.006 & 65.90 & 0.08 & 1631.8 & 5.8 & 0.22598 & 0.00042 & 0.18 & $\mathbf{a}$ \\
\hline 55.614 & 0.006 & 65.87 & 0.08 & 1903.8 & 5.8 & 0.22635 & 0.00039 & 0.17 & b \\
\hline 59.339 & 0.006 & 65.84 & 0.09 & 2169.6 & 5.8 & 0.22621 & 0.00037 & 0.16 & $\mathbf{a}$ \\
\hline 63.099 & 0.006 & 65.82 & 0.09 & 2436.7 & 5.8 & 0.22652 & 0.00036 & 0.16 & $\mathbf{b}$ \\
\hline 66.851 & 0.006 & 65.79 & 0.09 & 2701.6 & 5.8 & 0.22612 & 0.00035 & 0.15 & a \\
\hline 70.577 & 0.006 & 65.77 & 0.10 & 2962.8 & 5.8 & 0.22726 & 0.00035 & 0.15 & b \\
\hline 74.335 & 0.006 & 65.74 & 0.10 & 3224.5 & 5.8 & 0.22568 & 0.00034 & 0.15 & $\mathbf{a}$ \\
\hline 78.034 & 0.006 & 65.72 & 0.11 & 3480.4 & 5.8 & 0.22715 & 0.00034 & 0.15 & $\mathbf{b}$ \\
\hline 81.781 & 0.006 & 65.69 & 0.11 & 3738.6 & 5.8 & 0.22710 & 0.00034 & 0.15 & 8 \\
\hline 85.468 & 0.006 & 65.67 & 0.12 & 3992.0 & 5.8 & 0.22778 & 0.00034 & 0.15 & b \\
\hline 89.231 & 0.006 & 65.64 & 0.12 & 4251.0 & 5.8 & 0.23001 & 0.00035 & 0.15 & $\mathbf{a}$ \\
\hline 92.880 & 0.006 & 65.62 & 0.12 & 4503.0 & 5.8 & 0.23017 & 0.00034 & 0.15 & b \\
\hline
\end{tabular}


Table 10(a). Experimental liquid heat capacity data for HFC-32.

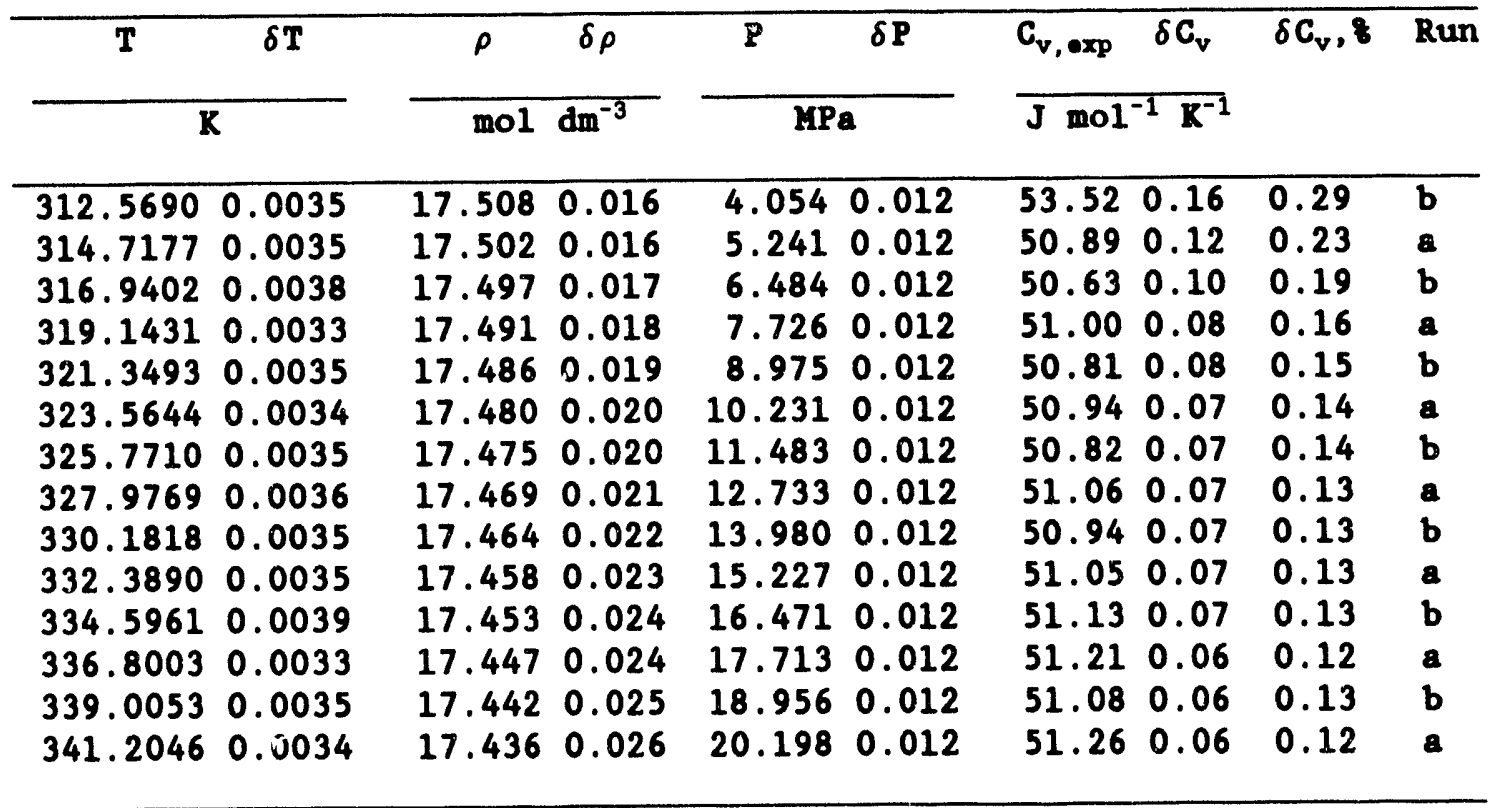

Table $10(b)$. Experimental liquid heat capacity data for HFC-32.

\begin{tabular}{|c|c|c|c|c|c|c|c|c|c|}
\hline $\mathbf{T}$ & $\delta \mathrm{T}$ & $\rho$ & $\delta \rho$ & $\mathbf{P}$ & $\delta P$ & $C_{v, \exp }$ & $\delta \mathrm{C}_{\mathrm{v}}$ & $\delta \mathrm{C}_{\mathrm{v}}, \mathrm{B}$ & $\overline{\text { Run }}$ \\
\hline \multicolumn{2}{|c|}{${ }^{\circ} \mathrm{P}$} & \multicolumn{2}{|c|}{$1 b \mathrm{ft}^{-3}$} & \multicolumn{2}{|c|}{ psia } & \multicolumn{4}{|c|}{ Btu $1 b^{-1} \cdot \mathrm{B}^{-1}$} \\
\hline $\begin{array}{l}102.954 \\
106.822 \\
110.822 \\
114.788 \\
118.759 \\
122.746 \\
126.718 \\
130.688 \\
134.657 \\
138.630 \\
142.603 \\
146.570 \\
150.539 \\
154.498\end{array}$ & $\begin{array}{l}0.006 \\
0.006 \\
0.007 \\
0.006 \\
0.006 \\
0.006 \\
0.006 \\
0.006 \\
0.006 \\
0.006 \\
0.007 \\
0.006 \\
0.006 \\
0.006\end{array}$ & $\begin{array}{l}56.86 \\
56.84 \\
56.83 \\
56.81 \\
56.79 \\
56.77 \\
56.75 \\
56.74 \\
56.72 \\
56.70 \\
56.68 \\
56.67 \\
56.65 \\
56.63\end{array}$ & $\begin{array}{l}0.05 \\
0.05 \\
0.06 \\
0.06 \\
0.06 \\
0.06 \\
0.07 \\
0.07 \\
0.07 \\
0.07 \\
0.08 \\
0.08 \\
0.08 \\
0.08\end{array}$ & $\begin{array}{r}587.9 \\
760.2 \\
940.5 \\
1120.6 \\
1301.7 \\
1484.0 \\
1665.5 \\
1846.8 \\
2027.7 \\
2208.4 \\
2388.9 \\
2569.0 \\
2749.3 \\
2929.5\end{array}$ & $\begin{array}{l}1.7 \\
1.7 \\
1.7 \\
1.7 \\
1.7 \\
1.7 \\
1.7 \\
1.7 \\
1.7 \\
1.7 \\
1.7 \\
1.7 \\
1.7 \\
1.7\end{array}$ & $\begin{array}{l}0.24587 \\
0.23379 \\
0.23260 \\
0.23430 \\
0.23342 \\
0.23403 \\
0.23348 \\
0.23456 \\
0.23403 \\
0.23452 \\
0.23492 \\
0.23527 \\
0.23469 \\
0.23549\end{array}$ & $\begin{array}{l}0.00071 \\
0.00054 \\
0.00045 \\
0.00038 \\
0.00036 \\
0.00033 \\
0.00032 \\
0.00031 \\
0.00030 \\
0.00030 \\
0.00031 \\
0.00029 \\
0.00029 \\
0.00029\end{array}$ & $\begin{array}{l}0.29 \\
0.23 \\
0.19 \\
0.16 \\
0.15 \\
0.14 \\
0.14 \\
0.13 \\
0.13 \\
0.13 \\
0.13 \\
0.12 \\
0.13 \\
0.12\end{array}$ & $\begin{array}{l}\text { b } \\
a \\
b \\
a \\
b \\
a \\
b \\
a \\
b \\
a \\
b \\
a \\
b \\
a\end{array}$ \\
\hline
\end{tabular}


Table 11(a). Experimental 1iquid heat capacity data for HFC-32.

\begin{tabular}{|c|c|c|c|c|c|c|c|c|}
\hline$T$ & $\delta \mathrm{T}$ & $\rho$ & $\delta \rho$ & $\mathbf{P}$ & $\delta P$ & $C_{v, \exp } \delta C_{v}$ & $\delta C_{v}, 8$ & Run \\
\hline \multicolumn{2}{|c|}{$\overline{\mathbf{K}}$} & mol & $d m^{-3}$ & \multicolumn{2}{|c|}{$\mathbf{M P a}$} & \multicolumn{3}{|l|}{$\mathrm{J} \mathrm{mol}^{-1} \mathrm{~K}^{-1}$} \\
\hline $\begin{array}{l}152.9366 \\
154.9359 \\
156.7176 \\
158.7148 \\
160.4805 \\
162.4557\end{array}$ & $\begin{array}{l}0.0029 \\
0.0029 \\
0.0030 \\
0.0029 \\
0.0030 \\
0.0030\end{array}$ & $\begin{array}{l}26.811 \\
26.793 \\
26.776 \\
26.758 \\
26.742 \\
26.724\end{array}$ & $\begin{array}{l}0.028 \\
0.033 \\
0.037 \\
0.042 \\
0.046 \\
0.051\end{array}$ & $\begin{array}{r}8.918 \\
13.597 \\
17.743 \\
22.343 \\
26.357 \\
30.788\end{array}$ & $\begin{array}{l}0.029 \\
0.029 \\
0.029 \\
0.029 \\
0.029 \\
0.029\end{array}$ & $\begin{array}{ll}53.60 & 0.19 \\
53.40 & 0.16 \\
53.31 & 0.15 \\
53.21 & 0.14 \\
53.16 & 0.14 \\
53.34 & 0.13\end{array}$ & $\begin{array}{l}0.35 \\
0.30 \\
0.28 \\
0.26 \\
0.26 \\
0.25\end{array}$ & $\begin{array}{l}\mathbf{a} \\
\mathbf{b} \\
\mathbf{a} \\
\mathbf{b} \\
\mathrm{a} \\
\mathrm{c}\end{array}$ \\
\hline
\end{tabular}

Table 11(b). Experimental liquid heat capacity data for HFC-32.

\begin{tabular}{|c|c|c|c|c|c|c|c|c|c|}
\hline $\mathbf{T}$ & $\delta T$ & $\rho$ & $\delta \rho$ & $\mathbf{P}$ & $\delta \mathbf{P}$ & $C_{v, \text { exp }}$ & $\delta \mathrm{C}_{\mathrm{v}}$ & $\delta C_{v}, 8$ & Run \\
\hline \multicolumn{2}{|c|}{${ }^{\circ} \mathbf{F}$} & \multicolumn{2}{|c|}{$1 \mathrm{~b} \mathrm{ft}^{-3}$} & \multicolumn{2}{|c|}{ psia } & \multicolumn{4}{|c|}{ Btu $1 b^{-1} \cdot{ }^{\circ} \mathrm{F}^{-1}$} \\
\hline $\begin{array}{l}-184.384 \\
-180.785 \\
-177.578 \\
-173.983 \\
-170.805 \\
-167.250\end{array}$ & $\begin{array}{l}0.005 \\
0.005 \\
0.005 \\
0.005 \\
0.005 \\
0.005\end{array}$ & $\begin{array}{l}87.08 \\
87.02 \\
86.96 \\
86.90 \\
86.85 \\
86.79\end{array}$ & $\begin{array}{l}0.09 \\
0.11 \\
0.12 \\
0.14 \\
0.15 \\
0.16\end{array}$ & $\begin{array}{l}1293.5 \\
1972.1 \\
2573.4 \\
3240.6 \\
3822.8 \\
4465.4\end{array}$ & $\begin{array}{l}4.3 \\
4.3 \\
4.3 \\
4.3 \\
4.3 \\
4.3\end{array}$ & $\begin{array}{l}0.24624 \\
0.24531 \\
0.24490 \\
0.24445 \\
0.24420 \\
0.24505\end{array}$ & $\begin{array}{l}0.00086 \\
0.00073 \\
0.00068 \\
0.00064 \\
0.00062 \\
0.00061\end{array}$ & $\begin{array}{l}0.35 \\
0.30 \\
0.28 \\
0.26 \\
0.26 \\
0.25\end{array}$ & $\begin{array}{l}a \\
b \\
a \\
b \\
a \\
c\end{array}$ \\
\hline
\end{tabular}


Table 12(a). Experimental liquid heat capacity data for HFC-32.

\begin{tabular}{|c|c|c|c|c|c|c|c|c|c|}
\hline $\mathbf{T}$ & $\delta T$ & $\rho$ & $\delta \rho$ & $\mathbf{P}$ & $\delta P$ & $\mathrm{C}_{\mathrm{v}, \exp }$ & $\delta \mathrm{C}_{\mathrm{v}}$ & $\delta C_{v}, 8$ & $\overline{\text { Run }}$ \\
\hline \multicolumn{2}{|c|}{$\overline{\mathbf{K}}$} & mol & $\mathrm{dm}^{-3}$ & \multicolumn{2}{|c|}{$\mathbf{M P a}$} & $\mathrm{J} \mathrm{mol}$ & ${ }^{-1} \mathrm{k}^{-1}$ & & \\
\hline $\begin{array}{l}166.0437 \\
169.8697 \\
173.6822 \\
177.4754\end{array}$ & $\begin{array}{l}0.0030 \\
0.0029 \\
0.0030 \\
0.0029\end{array}$ & $\begin{array}{l}26.184 \\
26.151 \\
26.119 \\
26.087\end{array}$ & $\begin{array}{l}0.026 \\
0.034 \\
0.042 \\
0.050\end{array}$ & $\begin{array}{r}7.359 \\
15.507 \\
23.506 \\
31.315\end{array}$ & $\begin{array}{l}0.009 \\
0.009 \\
0.009 \\
0.009\end{array}$ & $\begin{array}{l}52.34 \\
51.99 \\
51.86 \\
51.99\end{array}$ & $\begin{array}{l}0.20 \\
0.15 \\
0.13 \\
0.13\end{array}$ & $\begin{array}{l}0.39 \\
0.28 \\
0.26 \\
0.24\end{array}$ & $\begin{array}{l}a \\
a \\
a \\
a\end{array}$ \\
\hline
\end{tabular}

Table 12(b). Experimental 1iquid heat capacity data for HFC-32.

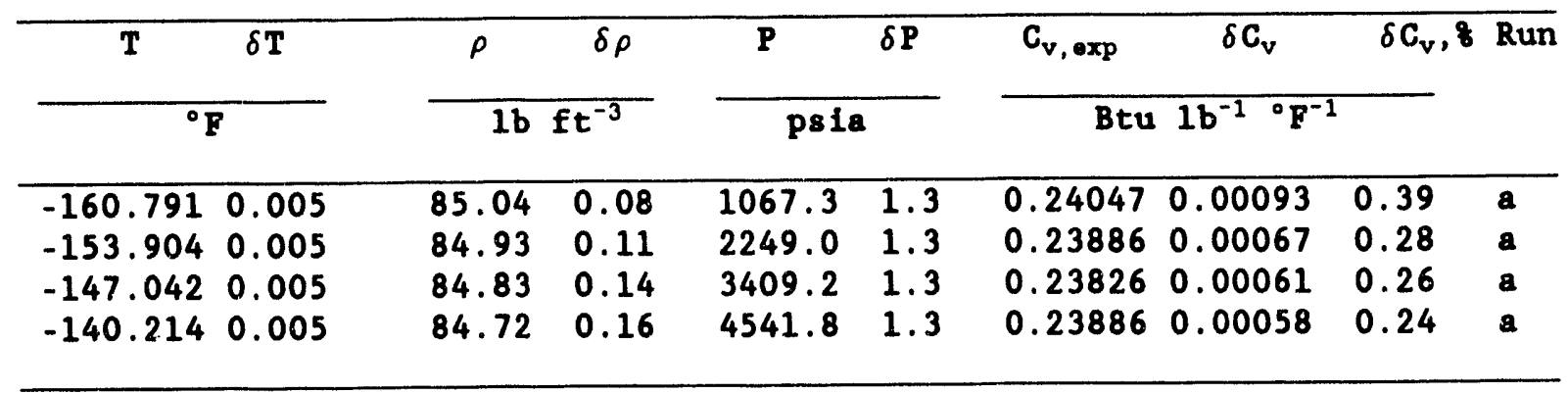


Table 13(a). Experimental liquid heat capacity data for HFC-32.

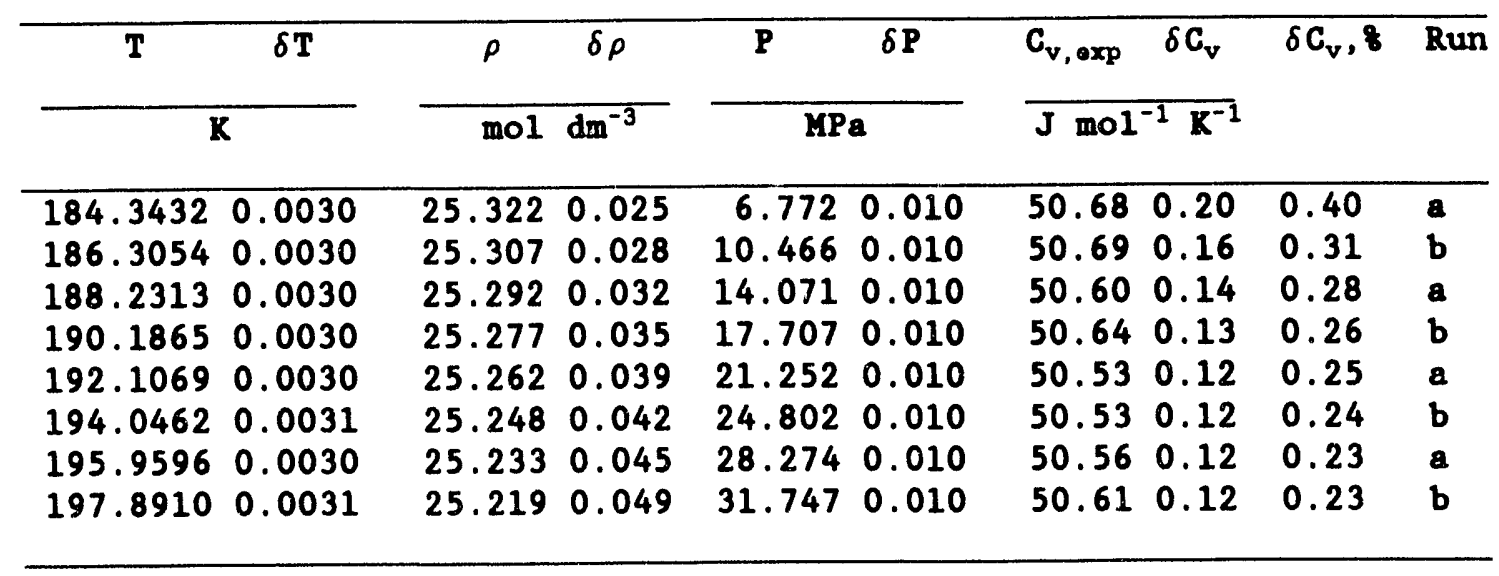

Table 13(b). Experimental 1iquid heat capacity data for HFC-32.

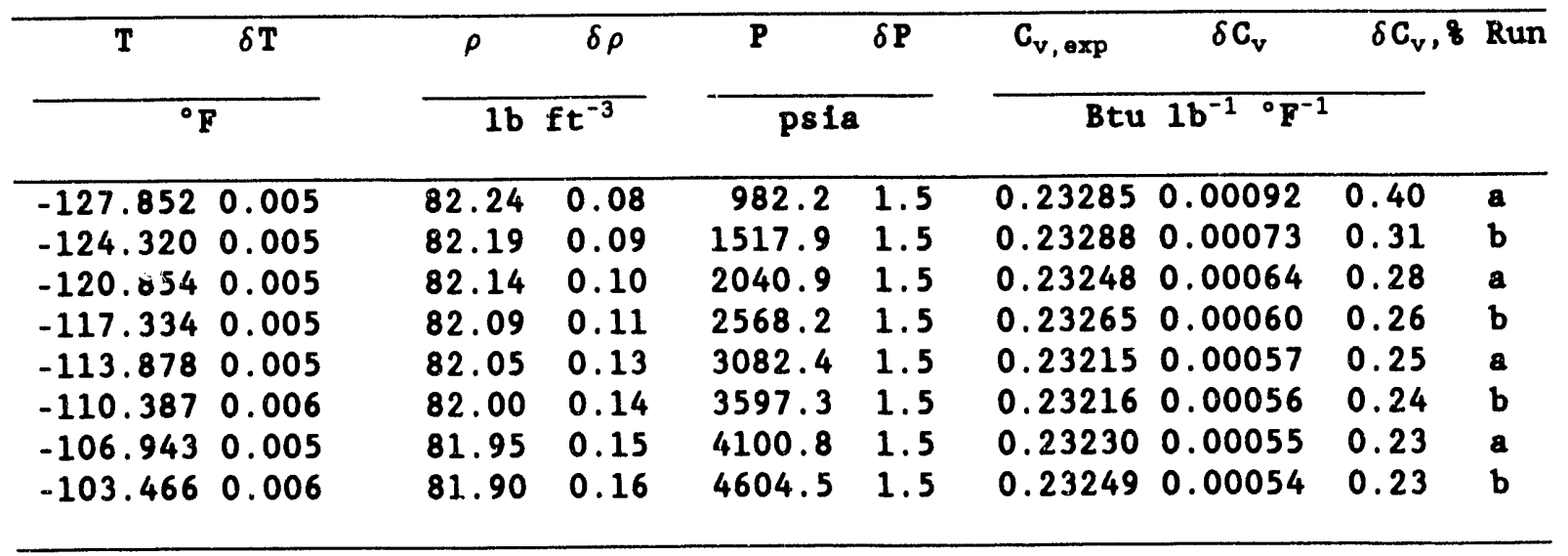


Table 14(a). Experimental two-phase heat capacity data for HFC-32.

\begin{tabular}{|c|c|c|c|c|c|c|c|c|c|c|}
\hline \multirow{2}{*}{$\mathrm{T}$} & \multirow[t]{2}{*}{$\delta \mathrm{T}$} & \multirow{2}{*}{$\begin{array}{c}\rho_{\sigma} \\
\text { mol } \mathrm{dm}^{-3}\end{array}$} & \multirow{2}{*}{$\begin{array}{l}\mathbf{P}_{\sigma} \\
\mathbf{M P a}\end{array}$} & $\mathrm{C}_{\mathrm{v}}^{(2)}$ & \multicolumn{3}{|c|}{$\delta \mathrm{C}_{\mathrm{v}}^{(2)} \delta \mathrm{C}_{\mathrm{v}}^{(2)}, 8 \mathrm{C}_{\sigma}$} & $\delta \mathrm{C}_{\sigma}$ & $\delta \mathrm{C}_{\sigma}, 8$ & \multirow[t]{2}{*}{ Run } \\
\hline & & & & & & & $\pi$ & & & \\
\hline 141.7357 & 0.0030 & 27.556 & 0.0001 & 82.41 & 0.06 & 0.08 & 82.41 & 0.06 & 0.08 & $\mathbf{a}$ \\
\hline 145.8061 & 0.0029 & 27.313 & 0.0002 & 82.07 & 0.06 & 0.07 & 82.06 & 0.06 & 0.07 & $\mathbf{a}$ \\
\hline 149.8458 & 0.0029 & 27.075 & 0.0003 & 82.03 & 0.06 & 0.08 & 82.02 & 0.06 & 0.08 & $\mathbf{a}$ \\
\hline 153.8710 & 0.0030 & 26.841 & 0.0005 & 81.83 & 0.06 & 0.08 & 81.81 & 0.06 & 0.08 & $\mathbf{a}$ \\
\hline 157.8716 & 0.0029 & 26.611 & 0.0008 & 81.75 & 0.06 & 0.08 & 81.72 & 0.06 & 0.08 & $\mathbf{a}$ \\
\hline 161.8533 & 0.0029 & 26.386 & 0.0012 & 81.61 & 0.06 & 0.08 & 81.58 & 0.06 & 0.08 & $\mathbf{a}$ \\
\hline 165.8155 & 0.0030 & 26.164 & 0.0018 & 81.16 & 0.06 & 0.08 & 81.12 & 0.06 & 0.08 & $a$ \\
\hline 169.7495 & 0.0030 & 25.946 & 0.0026 & 81.18 & 0.06 & 0.08 & 81.12 & 0.07 & 0.08 & $\mathbf{a}$ \\
\hline 173.6717 & 0.0029 & 25.730 & 0.0038 & 81.23 & 0.06 & 0.08 & 81.15 & 0.07 & 0.08 & $\mathbf{a}$ \\
\hline 177.5690 & 0.0030 & 25.518 & 0.0053 & 81.45 & 0.06 & 0.08 & 81.35 & 0.07 & 0.09 & $\mathbf{a}$ \\
\hline 181.4505 & 0.0030 & 25.308 & 0.0074 & 81.33 & 0.07 & 0.08 & 81.21 & 0.07 & 0.09 & $\mathbf{a}$ \\
\hline 185.3072 & 0.0030 & 25.100 & 0.0101 & 81.29 & 0.07 & 0.08 & 81.15 & 0.08 & 0.10 & $\mathbf{a}$ \\
\hline 189.1516 & 0.0030 & 24.895 & 0.0135 & 81.20 & 0.06 & 0.08 & 81.03 & 0.09 & 0.11 & $\mathbf{a}$ \\
\hline 192. & 0.0029 & 24.691 & 0.0179 & 81.39 & 0.06 & 0.08 & 81.19 & 0.09 & 0.11 & $\mathbf{a}$ \\
\hline 196.7737 & 0.0030 & 24.489 & 0.0233 & 81.33 & 0.07 & 0.08 & 81.09 & 0.10 & 0.13 & a \\
\hline 200.5483 & 0.0030 & 24.288 & 0.0299 & 81.55 & 0.07 & 0.08 & 81.27 & 0.11 & 0.14 & $\mathbf{a}$ \\
\hline 204.5556 & 0.0030 & 24.076 & 0.0385 & 81.80 & 0.06 & 0.07 & 81.48 & 0.12 & 0.15 & $\mathbf{a}$ \\
\hline 208.7849 & 0.0030 & 23.852 & 0.0498 & 82.07 & 0.06 & 0.07 & 81.71 & 0.14 & 0.17 & $\mathbf{a}$ \\
\hline 212.9828 & 0.0030 & 23.629 & 0.0635 & 82.34 & 0.06 & 0.07 & 81.92 & 0.16 & 0.19 & $\mathbf{a}$ \\
\hline 217 & 0.0030 & 23.406 & 0.0799 & 82.66 & 0.06 & 0.07 & 82.20 & 0.17 & 0.21 & $\mathbf{a}$ \\
\hline 221.3115 & 0.0030 & 23.184 & 0.0 & 82.91 & 0.06 & 0.08 & 82.40 & 0.19 & 0.24 & $\mathbf{a}$ \\
\hline 225.4342 & 0.0031 & 22.963 & 0.1226 & 83.27 & 0.06 & 0.08 & 82.72 & 0.21 & 0.26 & $\mathbf{a}$ \\
\hline 229.5376 & 0.0031 & 22.741 & 0.1496 & 83.58 & 0.07 & 0.08 & 82.99 & 0.23 & 0.28 & $\mathbf{a}$ \\
\hline 233.6094 & 0.0031 & 22.519 & 0.1808 & 84.01 & 0.07 & 0.08 & 83.37 & 0.26 & 0.31 & $\mathbf{a}$ \\
\hline 237.6613 & 0.0030 & 22.297 & 0.2167 & 84.57 & 0.07 & 0.08 & 83.90 & 0.28 & 0.33 & $\mathbf{a}$ \\
\hline 241.6846 & 0.0030 & 22.074 & 0.2577 & 85.05 & 0.07 & 0.08 & 84.36 & 0.30 & 0.35 & $\mathbf{a}$ \\
\hline 245.6850 & 0.0031 & 21.849 & 0.3042 & 85.38 & 0.07 & 0.08 & 84.68 & 0.31 & 0.37 & $\mathbf{a}$ \\
\hline 249.6596 & 0.0031 & 21.624 & 0.3566 & 85.90 & 0.07 & 0.08 & 85.20 & 0.33 & 0.39 & $\mathbf{a}$ \\
\hline 253.6128 & 0.0030 & 21.397 & 0.4153 & 86.31 & 0.07 & 0.08 & 5.63 & 0.35 & 0.41 & $\mathbf{a}$ \\
\hline 257.5 & 0.0 & 21. & 0.4807 & .74 & 0.07 & 0.08 & 6.10 & 0.36 & 0.42 & $\mathbf{a}$ \\
\hline 261. & 0.0 & 20. & 0.5532 & .28 & 0.07 & 0.08 & 6.69 & 0.38 & 0.43 & $\mathbf{a}$ \\
\hline 265.3195 & 0.0032 & 20.705 & 0.6332 & 88.21 & 0.07 & 0.08 & 87.70 & 0.39 & 0.44 & a \\
\hline 269.1857 & 0.0030 & 20.469 & 0.7215 & 88.67 & 0.07 & 0.08 & 88.27 & 0.39 & 0.44 & $\mathbf{a}$ \\
\hline 273.0159 & 0.0033 & 20.230 & 0.8178 & 89.41 & 0.08 & 0.09 & 89.16 & 0.39 & 0.44 & $\mathbf{a}$ \\
\hline 276.8306 & 0.0032 & 19.988 & 0.9230 & 90.05 & 0.08 & 0.09 & 89.97 & 0.39 & 0.44 & $\mathbf{a}$ \\
\hline 280.6208 & 0.0030 & 19.741 & 1.0374 & 90.93 & 0.08 & 0.08 & 91.07 & 0.39 & 0.43 & $\mathbf{a}$ \\
\hline 284.6251 & 0.0030 & 19.475 & 1.1694 & 91.68 & 0.07 & 0.08 & 92.11 & 0.38 & 0.41 & $\mathbf{a}$ \\
\hline 288.8352 & 0.0031 & 19.187 & 1.3213 & 92.66 & 0.07 & 0.08 & 93.47 & 0.36 & 0.39 & $\mathbf{a}$ \\
\hline 293.0161 & 0.0031 & 18.893 & 1.4863 & 93.41 & 0.07 & 0.08 & 94.68 & 0.34 & 0.36 & $\mathbf{a}$ \\
\hline 297.1666 & 0.0030 & 18.591 & 1.6648 & 94.70 & 0.08 & 0.08 & 96.54 & 0.31 & 0.32 & $\mathbf{a}$ \\
\hline 301.2947 & 0.0031 & 18.280 & 1.8577 & 95.73 & 0.08 & 0.08 & 98.28 & 0.28 & 0.29 & $\mathbf{a}$ \\
\hline 305.3859 & 0.0030 & 17.960 & 2.0648 & 96.80 & 0.08 & 0.08 & 100.15 & 0.28 & 0.28 & $\mathbf{a}$ \\
\hline
\end{tabular}


Table 14(b). Experimental two-phase heat capacity data for HFC-32.

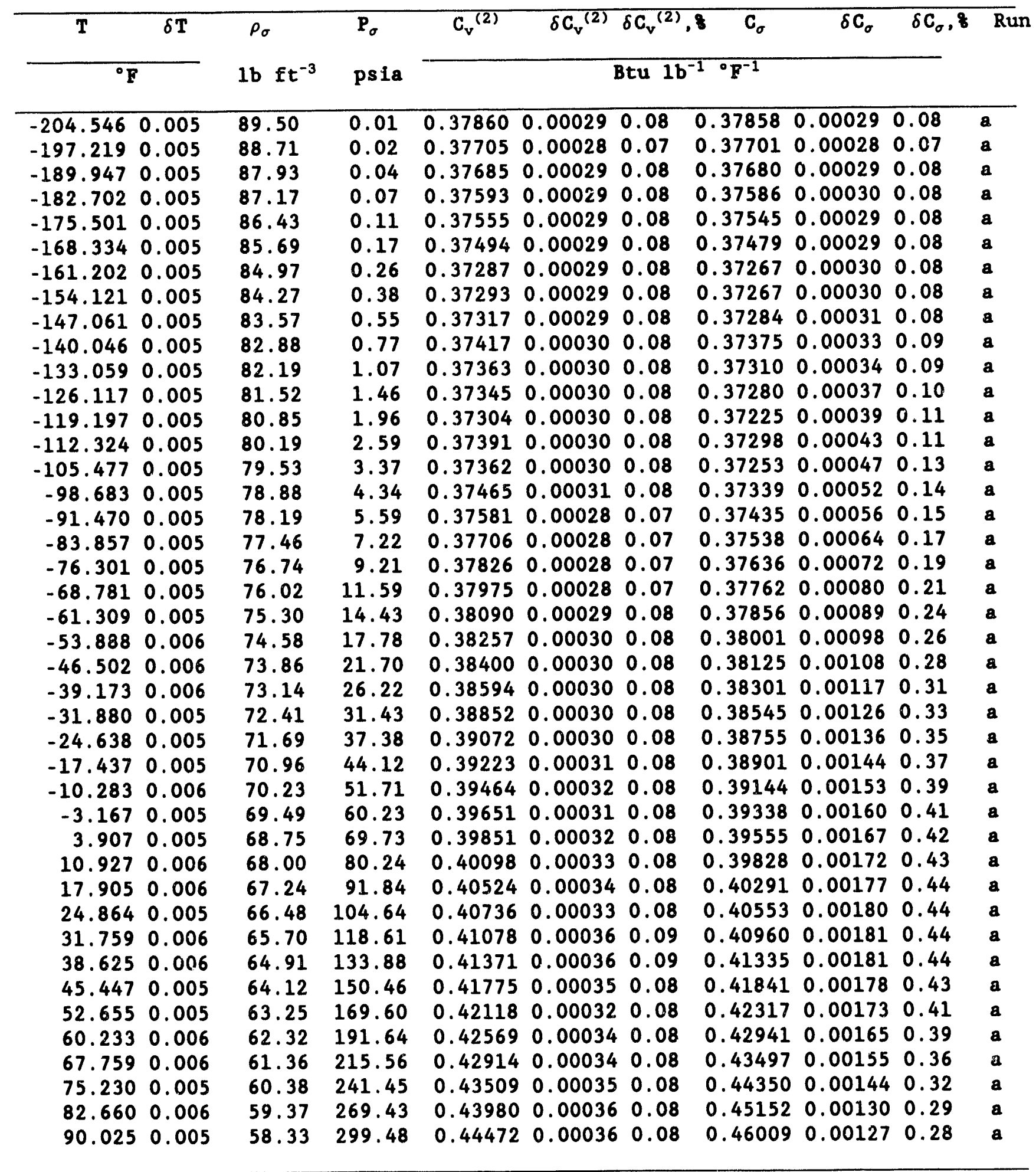


Table 15(a). Experimental two-phas heat capacity data for HFC-32.

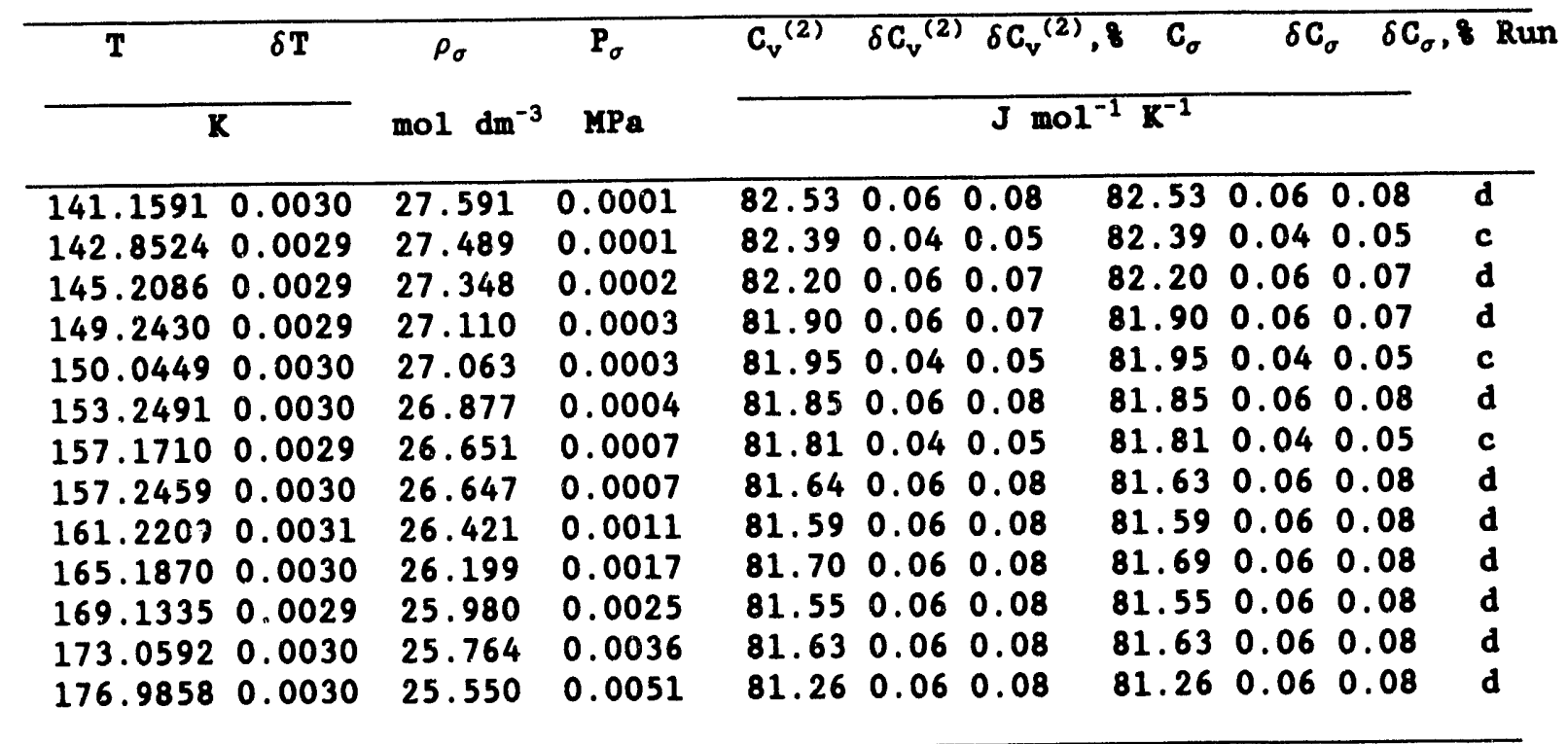

Table 15(b). Experimental two-phase heat capacity data for HFC-32.

\begin{tabular}{|c|c|c|c|c|c|c|c|c|c|c|}
\hline $\bar{T}$ & $\delta \mathbf{T}$ & \multirow{2}{*}{$\begin{array}{c}\rho_{\sigma} \\
1 b \quad f t^{-3}\end{array}$} & \multirow{2}{*}{$\begin{array}{l}\mathbf{P}_{\sigma} \\
\text { psia }\end{array}$} & $\mathrm{C}_{\mathrm{v}}{ }^{(2)}$ & \multicolumn{2}{|c|}{$\delta C_{v}{ }^{(2)} \delta C_{v}{ }^{(2)}, 8$} & $\mathbf{C}_{\sigma}$ & $\delta C_{\sigma}$ & $\delta \mathrm{C}_{\sigma}, 8$ & \multirow[t]{2}{*}{ Run } \\
\hline $\bar{E}$ & & & & & & Btu & ${ }^{\circ} \mathrm{F}^{-1}$ & & & \\
\hline $\begin{array}{l}-205.584 \\
-202.536 \\
-198.294 \\
-191.033 \\
-189.589 \\
-183.822 \\
-176.762 \\
-176.627 \\
-169.472 \\
-162.333 \\
-155.230 \\
-148.164 \\
-141.096\end{array}$ & $\begin{array}{l}0.005 \\
0.005 \\
0.005 \\
0.005 \\
0.005 \\
0.005 \\
0.005 \\
0.005 \\
0.005 \\
0.005 \\
0.005 \\
0.005 \\
0.005\end{array}$ & $\begin{array}{l}89.61 \\
89.28 \\
88.82 \\
88.05 \\
87.89 \\
87.29 \\
86.56 \\
86.54 \\
85.81 \\
85.09 \\
84.38 \\
83.67 \\
82.98\end{array}$ & $\begin{array}{l}0.01 \\
0.02 \\
0.02 \\
0.04 \\
0.04 \\
0.06 \\
0.10 \\
0.10 \\
0.16 \\
0.24 \\
0.36 \\
0.52 \\
0.74\end{array}$ & $\begin{array}{l}0.37916 \\
0.37850 \\
0.37764 \\
0.37628 \\
0.37649 \\
0.37602 \\
0.37585 \\
0.37505 \\
0.37484 \\
0.37532 \\
0.37466 \\
0.37503 \\
0.37331\end{array}$ & $\begin{array}{l}0.00029 \\
0.00017 \\
0.00028 \\
0.00028 \\
0.00017 \\
0.00029 \\
0.00017 \\
0.00029 \\
0.00030 \\
0.00029 \\
0.00029 \\
0.00029 \\
0.00029\end{array}$ & $\begin{array}{l}0.08 \\
0.05 \\
0.07 \\
0.07 \\
0.05 \\
0.08 \\
0.05 \\
0.08 \\
0.08 \\
0.08 \\
0.08 \\
0.08 \\
0.08\end{array}$ & $\begin{array}{l}0.37915 \\
0.37850 \\
0.37763 \\
0.37628 \\
0.37649 \\
0.37602 \\
0.37584 \\
0.37504 \\
0.37483 \\
0.37532 \\
0.37466 \\
0.37504 \\
0.37333\end{array}$ & $\begin{array}{l}0.00029 \\
0.00017 \\
0.00028 \\
0.00028 \\
0.00017 \\
0.00029 \\
0.00017 \\
0.00029 \\
0.00030 \\
0.00029 \\
0.00029 \\
0.00029 \\
0.00029\end{array}$ & $\begin{array}{l}0.08 \\
0.05 \\
0.07 \\
0.07 \\
0.05 \\
0.08 \\
0.05 \\
0.08 \\
0.08 \\
0.08 \\
0.08 \\
0.08 \\
0.08\end{array}$ & $\begin{array}{l}\text { d } \\
c \\
d \\
d \\
c \\
d \\
c \\
d \\
d \\
d \\
d \\
d \\
d\end{array}$ \\
\hline
\end{tabular}


Table 16(a). Experimental two-phase heat capacity data for HFC-32.

\begin{tabular}{|c|c|c|c|c|c|c|c|c|c|c|}
\hline $\mathbf{T}$ & $\delta T$ & & $\mathbf{P}_{\sigma}$ & $\mathrm{c}_{\mathrm{v}}{ }^{(2)}$ & $\delta C_{v}$ & & $\mathbf{C}_{\sigma}$ & $\overline{\bar{C}_{\sigma}}$ & $\sigma$ & \\
\hline & 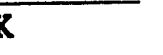 & 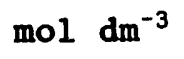 & $\mathrm{MPa}$ & & & & & & & \\
\hline 144. & 0.0030 & 27.386 & 0.0001 & 2.33 & 0.05 & & 82.32 & 0.05 & 0.07 & a \\
\hline & & 27.094 & 0.0003 & $1.96 \mathrm{C}$ & 0.05 & 0.07 & 81.94 & 0.06 & 0.07 & $\mathbf{a}$ \\
\hline 51 & 29 & 26.809 & 0.0005 & 81.76 & 0.05 & 0.07 & 81.72 & 0.06 & 0.07 & $\mathbf{a}$ \\
\hline & 30 & 26.530 & 0.0009 & 81.66 & 0.06 & 0.07 & 81.60 & 0.06 & 0.07 & $a$ \\
\hline & 0.0030 & 26.258 & 0.0015 & 81.51 & 0.06 & 0.07 & 81.42 & 0.06 & 0.08 & $\mathbf{a}$ \\
\hline & 0.0030 & 25.991 & 0.0024 & 81.51 & 0.06 & 0.07 & 81.38 & 0.07 & 0.08 & $\mathbf{a}$ \\
\hline & 0.0030 & 25. & 0.0 & 81.54 & 0.06 & & 1.36 & 0.08 & 0.10 & $\mathbf{a}$ \\
\hline 73 & 0.0030 & 25. & & .61 & 0.06 & 0.07 & 81.37 & 0.09 & 0.11 & $\mathbf{a}$ \\
\hline 37 & 0. & 25. & 0 . & .56 & 0.06 & 0.07 & 81.25 & 0.11 & 0.14 & $\mathbf{a}$ \\
\hline 83 & 0. & 24. & & .54 & 0.06 & 0.07 & 81.14 & 0.14 & 0.17 & $\mathbf{a}$ \\
\hline 83 & 0. & 24 & & 81.71 & 0.06 & 0.07 & 81.20 & 0.17 & 0.21 & $\mathbf{a}$ \\
\hline & 0. & & 0. & 82.11 & 0.06 & 0.07 & 81.48 & 0.21 & 0.25 & a \\
\hline 2 & 0. & 24 & 0. & 82.06 & 0.06 & 0.07 & 81.29 & 0.25 & 0.30 & $\mathbf{a}$ \\
\hline 39 & 0. & & 0. & .59 & 0.06 & 0 . & 81.67 & 0.30 & 0.36 & a \\
\hline & & & & .23 & 0.06 & 0. & 82.13 & 0.35 & 0.43 & a \\
\hline & & & & .54 & 0.06 & 0 . & 82.26 & 0.41 & 0.50 & a \\
\hline & 0. & & & .75 & 0.06 & 0 & 82.27 & 0.47 & 0.58 & $\mathbf{a}$ \\
\hline & 0.0 & 23 & & .74 & 0.07 & 0.08 & 82.05 & 0.54 & 0.66 & $\mathbf{a}$ \\
\hline .08 & 0.1 & 22 & & .23 & 0.07 & 0.08 & 82.32 & 0.62 & 0.75 & $\mathbf{a}$ \\
\hline 60 & 0. & 22 & & 85.01 & 0.07 & 0.08 & 82.87 & 0.70 & 0.84 & $\mathbf{a}$ \\
\hline 5 & 0. & & 0 & 85.94 & 0.07 & 0.08 & 83.58 & 0.78 & 0.93 & b \\
\hline & 0. & 22 & 0 & 86.25 & 0.07 & 0.08 & 83.81 & 0.80 & 0.96 & c \\
\hline 6 & 0. & & & 86.41 & 0.07 & 0 . & 83.81 & 0.86 & 1.03 & b \\
\hline 97 & 0 . & 22 & & 86.71 & 0.07 & 0. & 4.03 & 0.89 & 1.06 & c \\
\hline 710 & & & & 87.50 & 0.07 & 0. & 4.57 & 0.98 & 1.16 & c \\
\hline & 0.0032 & 21. & & 88.40 & 0.07 & 0. & 5.24 & 1.07 & 1.26 & c \\
\hline & & & & .13 & 0.07 & 0.08 & 85.74 & 1.16 & 1.36 & c \\
\hline .8504 & $0 . C$ & & & .09 & 0.07 & 0. & 86.47 & 1.26 & 1.45 & c \\
\hline .9482 & 0.0 & & & .95 & 0.08 & 0.08 & 87.12 & 1.35 & 1.55 & c \\
\hline & 0. & & 0 & 92.26 & 0.08 & 0.08 & 88.23 & 1.45 & 1.64 & c \\
\hline & 0. & & & 92.76 & 0.08 & 0.09 & 88.56 & 1.54 & 1.74 & c \\
\hline & 0. & & & 93.83 & 0.08 & 0.09 & 89.48 & 1.64 & 1.83 & c \\
\hline & 0. & & & 95.33 & 0.09 & 0.09 & 90.87 & 1.73 & 1.90 & c \\
\hline & 0. & 15 & & 96.42 & 0.09 & 0.09 & 91.87 & 1.82 & 1.98 & c \\
\hline & 0. & & 1. & 97.41 & 0.09 & 0. & 92.83 & 1.91 & 2.06 & c \\
\hline 18 & 0.0 & 19. & 1.3 & 98.87 & 0.09 & & 94.31 & 1.99 & 2.11 & c \\
\hline & 0.0 & & 1.5 & 100.14 & 0.09 & & 95.67 & 2.07 & 2.17 & c \\
\hline & 0.0 & & & .41 & 0.09 & & 10 & 2.15 & 2.21 & c \\
\hline 3528 & 0.00 & & & .37 & 0.10 & & 99.38 & 2.20 & 2.21 & c \\
\hline 306.1487 & 0.00 & & & .91 & 0. & & 101.22 & 2.28 & 2.25 & c \\
\hline & 0.0 & & & .05 & 0.11 & 0. & 102.92 & 2.32 & 2.25 & c \\
\hline & 0.0 & & & 107.36 & 0.11 & & 104.86 & 2.38 & 2.27 & c \\
\hline & 0.0 & & & 109.16 & 0.12 & & 107.54 & 2.41 & 2.25 & c \\
\hline & 0.0 & & 3 & 108.38 & 3.84 & & 107.85 & 4.55 & 4.22 & c \\
\hline & & & & 113.01 & 0.12 & & 114.03 & 2.45 & 2.14 & c \\
\hline .2040 & 0.00 & 15.859 & & 115.64 & 0.13 & 0.11 & 118.61 & 2.45 & 2.06 & c \\
\hline 331.7698 & 0.00 & & & 118.15 & 0.13 & 0.11 & 123.73 & 2.42 & 1.96 & c \\
\hline & 0.0 & & & 120.84 & 0.14 & 0.11 & 129.96 & 2.38 & 1.83 & c \\
\hline 338.7664 & 0.003 & & 4.4700 & 123.92 & 0.14 & 0.1 & 138.00 & 2.34 & 1.70 & c \\
\hline 342.1731 & 0.0032 & 14.048 & 4.8020 & 128.97 & 0.14 & 0.11 & 150.25 & 2.35 & 1.57 & c \\
\hline
\end{tabular}


Table 16(b). Experimental two-phase heat capacity data for HFC-32.

\begin{tabular}{|c|c|c|c|c|c|c|c|c|c|c|}
\hline $\mathbf{T}$ & $\delta \mathrm{T}$ & $\rho_{0}$ & $\mathbf{P}_{\sigma}$ & $C_{v}{ }^{(2)}$ & $\delta C_{v}{ }^{(2)}$ & $\delta C_{v}{ }^{2}$ & $\mathbf{C}_{\sigma}$ & $\delta \mathrm{C}_{\sigma}$ & $\delta \mathrm{C}_{\sigma}$ & \\
\hline${ }^{\circ} \mathbf{F}$ & & $1 \mathrm{~b} f t$ & pold & & & Btu & ${ }^{-1} \cdot{ }^{\circ} F^{-1}$ & & & \\
\hline-199.437 & 0.005 & 88.94 & 0.02 & 0.37824 & 0.00025 & 0.07 & 0.37818 & 0.00025 & 0.07 & $\mathbf{a}$ \\
\hline-190.527 & 0.005 & 87.99 & 0.04 & 0.37655 & 0.00025 & 0.07 & 0.37644 & 0.00025 & 0.07 & a \\
\hline-181.705 & 0.005 & 87.07 & 0.07 & 0.37561 & 0.00025 & 0.07 & 0.37543 & 0.00026 & 0.07 & $\mathbf{a}$ \\
\hline-172.934 & 0.005 & 86.16 & 0.13 & 0.37514 & 0.00026 & 0.07 & 0.37487 & 0.00027 & 0.07 & $\mathbf{a}$ \\
\hline-164.237 & 0.005 & 85.28 & 0.22 & 0.37447 & 0.00026 & 0.07 & 0.37406 & 0.00029 & 0.08 & $\mathbf{a}$ \\
\hline-155.607 & 0.005 & 84.41 & 0.35 & 0.37447 & 0.00026 & 0.07 & 0.37389 & 0.00031 & 0.08 & a \\
\hline-147.040 & 0.005 & 83.56 & 0.55 & 0.3 & 0.00026 & 0.07 & 0.37379 & 0.00036 & 0.10 & a \\
\hline-138.537 & 0.005 & 82 & 3 & 491 & 0.00026 & 0.07 & 0.37382 & 0.00043 & 0.11 & a \\
\hline-130.101 & 0.006 & 81.91 & 1.23 & 0.37470 & 0.00027 & 0.07 & 0.37327 & 0.00052 & 0.14 & a \\
\hline-121.723 & 0.005 & 81.10 & 1.77 & 0.37461 & 0.00027 & 0.07 & 0.37276 & 0.00063 & 0.17 & $\mathbf{a}$ \\
\hline-113.407 & 0.005 & 80.29 & 2.48 & 0.37537 & 0.00027 & 0.07 & 0.37303 & 0.00077 & 0.21 & $\mathbf{a}$ \\
\hline-105.147 & 0.005 & 79.50 & 3.42 & 0.37721 & 0.00027 & 0.07 & 0.37431 & 0.00094 & 0.25 & $\mathbf{a}$ \\
\hline-96.947 & 0.005 & 78.72 & 4.61 & 0.37699 & 0.00027 & 0.07 & 0.37346 & 0.00114 & 0.30 & $\mathbf{a}$ \\
\hline-88.827 & 0.005 & 77.94 & 6.12 & 0.37943 & 0.00028 & 0.07 & 0.37519 & 0.00136 & 0.36 & a \\
\hline-80.750 & 0.006 & 77.17 & 7.99 & 0.38236 & 0.00028 & 0.07 & 0.37733 & 0.00161 & 0.43 & $\mathbf{a}$ \\
\hline-72.734 & 0.006 & 76.40 & 10.28 & 0.38381 & 0.00029 & 0.07 & 0.37793 & 0.00188 & 0.50 & a \\
\hline-64.783 & 0.006 & 75.63 & 13.05 & 0.38475 & 0.00029 & 0.08 & 0.37796 & 0.00218 & 0.58 & a \\
\hline-56. & 0.006 & 74.87 & 16.36 & 0.38473 & 0.00030 & 0.08 & 0.37697 & 0.00250 & 0.66 & a \\
\hline-49.071 & 0.006 & 74.11 & 20.26 & 0.38695 & 0.00031 & 0.08 & 0.37818 & 0.00284 & 0.75 & $\mathbf{a}$ \\
\hline-41.285 & 0.006 & 73 & 24.85 & 0.39056 & 031 & 0.08 & 0.38073 & 0.0 & 0.84 & $\mathbf{a}$ \\
\hline-33.829 & 0.006 & 72.61 & 29.97 & 0.39484 & 0.00031 & 0.08 & 0.38396 & 0.00357 & 0.93 & b \\
\hline-31.483 & 0.006 & 72.37 & 31.74 & 0.39625 & 0.00031 & 0.08 & 0.38504 & 0.00368 & 0.96 & c \\
\hline-26.162 & 0.006 & 71.84 & 36.06 & 0.39699 & 0.00032 & 0.08 & 0.38501 & 0.00396 & 1.03 & b \\
\hline-23.819 & 0.006 & 71.61 & 38.10 & 0.39836 & 0.00031 & 0.08 & 0.38605 & 0.00408 & 1.06 & c \\
\hline-16.202 & 0.006 & 70.84 & 45.36 & 0.40197 & 0.00032 & 0.08 & 0.38854 & 0.00450 & 1.16 & c \\
\hline-8.664 & 0.006 & 70.06 & 53.57 & 0.40614 & 0.00033 & 0.08 & 0.39161 & 0.00492 & 1.26 & c \\
\hline-1.181 & 0.006 & 69.29 & 62.79 & 0.40950 & 0.00034 & 0.08 & 0.39390 & 0.00534 & 1.36 & c \\
\hline 6.261 & 0.006 & 68.50 & 73.13 & 0.41389 & 0.00034 & 0.08 & 0.39726 & 0.00578 & 1.45 & c \\
\hline 13.637 & 0.006 & 67.71 & 84.60 & 0.41786 & 0.00035 & 0.08 & 0.40026 & 0.00622 & 1.55 & c \\
\hline 20.956 & 0.006 & 66.91 & 97.30 & 0.42384 & 0.0 & 0.08 & 0.40534 & 0.00666 & 1.64 & c \\
\hline 28. & 0.0 & 66.10 & 111.33 & 0.42614 & 0.0 & 0.09 & 0.40684 & 0.00709 & 1.74 & c \\
\hline 35. & 0.0 & 65.28 & 126.63 & 0.4 & 0.0 & 0.09 & 0.41108 & 0.00752 & 1.83 & c \\
\hline 42. & 0.0 & 64.45 & 143.38 & 0.4 & 0.0 & 0.09 & 0.4 & 0.0 & 1.90 & c \\
\hline 49.722 & 0.006 & 63.60 & 161.61 & 0.44296 & 0.00039 & 0.09 & 0.42209 & 0.00836 & 1.98 & c \\
\hline 56.794 & 0.006 & 62.74 & 181.39 & 0.44751 & 0.00042 & 0.09 & 0.42648 & 0.00877 & 2.06 & c \\
\hline 63.809 & 0.006 & 61.87 & 202.74 & 0.45421 & 0.00041 & 0.09 & 0.43328 & 0.00915 & 2.11 & c \\
\hline 70.775 & 0.006 & 60.97 & 225.75 & 0.46008 & 0.00043 & 0.09 & 0.43954 & 0.00952 & 2.17 & c \\
\hline 77.697 & 0.006 & 60.05 & 250.49 & 0.46589 & 0.00042 & 0.09 & 0.44610 & 0.00986 & 2.21 & c \\
\hline 84.565 & 0.006 & 59.11 & 276.98 & 0.47488 & 0.00044 & 0.09 & 0.45655 & 0.01010 & 2.21 & c \\
\hline 91.398 & 0.006 & 58.13 & 305.34 & 0.48198 & 0.00048 & 0.10 & 0.46504 & 0.01048 & 2.25 & c \\
\hline 98.169 & 0.006 & 57.13 & 335.54 & 0.48719 & 0.00049 & 0.10 & 0.47283 & 0.01065 & 2.25 & c \\
\hline 104.897 & 0.006 & 56.09 & 367.69 & 0.49322 & 0.00051 & 0.10 & 0.48174 & 0.01093 & 2.27 & c \\
\hline 111.549 & 0.006 & 55.02 & 401.70 & 0.50151 & 0.00053 & 0.11 & 0.49407 & 0.01109 & 2.25 & c \\
\hline 118.114 & 0.006 & 53.91 & 437.53 & 0.49791 & 0.01764 & 3.54 & 0.49550 & 0.02092 & 4.22 & c \\
\hline 124.586 & 0.006 & 52.75 & 475.15 & 0.51919 & 0.00055 & 0.11 & 0.52387 & 0.01124 & 2.14 & c \\
\hline 131.097 & 0.006 & 51.51 & 515.45 & 0.53129 & 0.00060 & 0.11 & 0.54491 & 0.01124 & 2.06 & c \\
\hline 137.516 & 0.006 & 50.20 & 557.68 & 0.54281 & 0.00059 & 0.11 & 0.56843 & 0.01113 & 1.96 & c \\
\hline 143.859 & 0.006 & 48.80 & 602.00 & 0.55514 & 0.00063 & 0.11 & 0.59707 & 0.01096 & 1.83 & c \\
\hline 150.110 & 0.006 & 47.28 & 648.32 & 0.56930 & 0.00062 & 0.11 & 0.63399 & 0.01077 & 1.70 & c \\
\hline 156.242 & 0.006 & 45.62 & 696.48 & 0.59249 & 0.00064 & 0.11 & 0.69025 & 0.01081 & 1.57 & c \\
\hline
\end{tabular}


Table 17a. Compressed liquid density measurements for HCFC-124 (SI units).

$\begin{array}{lrr}\text { T/K } & \text { rho/g/cc } & \text { P/kPa } \\ 275.247 & 1.4312 & 396.9 \\ 275.196 & 1.4350 & 493.1 \\ 275.202 & 1.4354 & \text { f03.5 } \\ 275.195 & 1.4369 & 1050.7 \\ 275.213 & 1.4346 & 1575.7 \\ 275.159 & 1.4366 & 2058.1 \\ 275.146 & 1.4380 & 2469.2 \\ 275.188 & 1.4398 & 3036.4 \\ 275.175 & 1.4417 & 3592.0 \\ 275.071 & 1.4436 & 3951.7 \\ 275.123 & 1.4449 & 4507.3 \\ 275.180 & 1.4464 & 4948.2 \\ 275.143 & 1.4480 & 5470.6 \\ 275.606 & 1.4480 & 6023.4 \\ 275.219 & 1.4505 & 6361.0 \\ & & \\ 278.752 & 1.4202 & 406.6 \\ 278.779 & 1.4236 & 493.6 \\ 278.700 & 1.4246 & 604.6 \\ 278.699 & 1.4258 & 1050.3 \\ 278.702 & 1.4238 & 1578.8 \\ 278.723 & 1.4256 & 2060.2 \\ 278.695 & 1.4272 & 2474.9 \\ 278.725 & 1.4291 & 3039.7 \\ 278.697 & 1.4311 & 3600.1 \\ 278.714 & 1.4324 & 3957.9 \\ 278.669 & 1.4344 & 4514.5 \\ 278.702 & 1.4358 & 4944.6 \\ 278.740 & 1.4374 & 5475.5 \\ 278.763 & 1.4391 & 6022.7 \\ 278.733 & 1.4403 & 6371.2 \\ & & \\ 283.658 & 1.4043 & 410.6 \\ 283.727 & 1.4077 & 494.0 \\ 283.630 & 1.4086 & 605.9 \\ 283.621 & 1.4100 & 1051.1 \\ 283.635 & 1.4082 & 1582.6 \\ 283.631 & 1.41 C 1 & 2063.0 \\ 283.609 & 1.4119 & 2480.0 \\ 283.647 & 1.4139 & 3044.1 \\ 283.636 & 1.4161 & 3608.2 \\ 283.657 & 1.4174 & 3964.2 \\ 283.617 & 1.4196 & 4522.8 \\ 283.625 & 1.4211 & 4948.2 \\ 283.640 & 1.4229 & 5483.9 \\ 283.656 & 1.4247 & 6029.4 \\ 283.647 & 1.4260 & 6384.5 \\ 288.544 & 1.3882 & 412.6\end{array}$




$\begin{array}{lrr}288.611 & 1.3916 & 494.6 \\ 288.538 & 1.3924 & 607.6 \\ 288.526 & 1.3941 & 1052.6 \\ 288.538 & 1.3924 & 1585.4 \\ 288.540 & 1.3944 & 2065.9 \\ 288.490 & 1.3964 & 2486.7 \\ 288.538 & 1.3985 & 3043.9 \\ 288.568 & 1.4007 & 3614.7 \\ 288.549 & 1.4022 & 3961.5 \\ 288.514 & 1.4046 & 4531.6 \\ 288.529 & 1.4062 & 4957.1 \\ 288.551 & 1.4082 & 5495.6 \\ 288.543 & 1.4102 & 6038.9 \\ 288.555 & 1.4114 & 6395.6 \\ & & \\ 293.534 & 1.3713 & 413.8 \\ 293.555 & 1.3748 & 495.6 \\ 293.482 & 1.3756 & 608.9 \\ 293.483 & 1.3776 & 1054.3 \\ 293.483 & 1.3761 & 1589.4 \\ 293.517 & 1.3760 & 1589.8 \\ 293.488 & 1.3782 & 2067.2 \\ 293.525 & 1.3802 & 2492.4 \\ 293.475 & 1.3827 & 3045.9 \\ 293.516 & 1.3851 & 3624.9 \\ 293.484 & 1.3867 & 3958.4 \\ 293.468 & 1.3892 & 4540.6 \\ 293.473 & 1.3909 & 4965.8 \\ 293.489 & 1.3931 & 5505.2 \\ 293.490 & 1.3952 & 6049.3 \\ 293.490 & 1.3966 & 6410.0 \\ 298.571 & 1.1061 & 430.3 \\ 298.546 & 1.3574 & 496.5 \\ 298.475 & 1.3581 & 610.4 \\ 298.468 & 1.3606 & 1056.2 \\ 298.477 & 1.3616 & 2069.8 \\ 298.520 & 1.3636 & 2497.5 \\ 298.471 & 1.3664 & 3051.1 \\ 298.510 & 1.3690 & 3629.1 \\ 298.471 & 1.3707 & 3968.2 \\ 298.464 & 1.3734 & 4551.2 \\ 298.466 & 1.375 . & 4973.2 \\ 298.481 & 1.3776 & 5513.0 \\ 298.486 & 1.3799 & 6059.0 \\ 298.487 & 1.3813 & 5425.9 \\ & & \\ 303.543 & 1.2562 & 516.7 \\ 303.470 & 1.3403 & 612.1 \\ 303.482 & 1.3368 & 726.6 \\ 303.472 & 1.3432 & 1058.4 \\ 303.488 & 1.3392 & 1149.6 \\ 303.499 & 1.3418 & 1596.2 \\ 303.475 & 1.3445 & 2073.3\end{array}$




\begin{tabular}{|c|c|c|}
\hline $\begin{array}{l}303.515 \\
303.467 \\
303.500 \\
303.473 \\
303.459 \\
303.468 \\
303.479 \\
303.488 \\
303.492\end{array}$ & $\begin{array}{l}1.3467 \\
1.3497 \\
1.3525 \\
1.3544 \\
1.3573 \\
1.3593 \\
1.3618 \\
1.3642 \\
1.3658\end{array}$ & $\begin{array}{l}2503.3 \\
3056.8 \\
3635.5 \\
3974.1 \\
4563.1 \\
4979.2 \\
5519.9 \\
6070.1 \\
6441.2\end{array}$ \\
\hline $\begin{array}{l}308.372 \\
308.377 \\
308.390 \\
308.377 \\
308.416 \\
308.366 \\
308.405 \\
308.373 \\
308.376 \\
308.373 \\
308.387 \\
308.391 \\
308.394\end{array}$ & $\begin{array}{l}1.3223 \\
1.3259 \\
1.3274 \\
1.3273 \\
1.3297 \\
1.3330 \\
1.3360 \\
1.3380 \\
1.3412 \\
1.3433 \\
1.3460 \\
1.3486 \\
1.3504\end{array}$ & $\begin{array}{r}613.4 \\
1060.6 \\
1599.5 \\
2077.2 \\
2508.9 \\
3062.3 \\
3644.8 \\
3979.3 \\
4576.8 \\
4986.7 \\
5538.0 \\
6081.2 \\
6453.1\end{array}$ \\
\hline $\begin{array}{l}313.327 \\
313.338 \\
313.328 \\
313.331 \\
313.336 \\
313.395 \\
313.364 \\
313.360 \\
313.363 \\
313.373 \\
313.396 \\
313.352\end{array}$ & $\begin{array}{l}1.3066 \\
1.3098 \\
1.3131 \\
1.3164 \\
1.3192 \\
1.3220 \\
1.3219 \\
1.3246 \\
1.3272 \\
1.3310 \\
1.3359 \\
1.3354\end{array}$ & $\begin{array}{l}1094.1 \\
1559.9 \\
2062.6 \\
2596.0 \\
3044.4 \\
3568.6 \\
4091.3 \\
4537.0 \\
4997.2 \\
5590.8 \\
6012.6 \\
6520.7\end{array}$ \\
\hline $\begin{array}{l}318.224 \\
318.252 \\
318.234 \\
318.236 \\
318.256 \\
318.304 \\
318.287 \\
318.277 \\
318.283 \\
318.283 \\
318.300 \\
318.271\end{array}$ & $\begin{array}{l}1.2876 \\
1.2911 \\
1.2948 \\
1.2985 \\
1.3014 \\
1.3046 \\
1.3047 \\
1.3077 \\
1.3105 \\
1.3146 \\
1.3165 \\
1.3193\end{array}$ & $\begin{array}{l}1097.2 \\
1568.3 \\
2068.7 \\
2612.2 \\
3052.0 \\
3582.8 \\
4103.8 \\
4549.1 \\
5017.2 \\
5604.9 \\
6023.7 \\
6533.0\end{array}$ \\
\hline $\begin{array}{l}323.093 \\
323.134 \\
323.094 \\
323.107\end{array}$ & $\begin{array}{l}1.2681 \\
1.271 .8 \\
1.2760 \\
1.2800\end{array}$ & $\begin{array}{l}1099.9 \\
1572.8 \\
2073.2 \\
2622.3\end{array}$ \\
\hline
\end{tabular}




$\begin{array}{lll}323.137 & 1.2833 & 3060.2 \\ 323.121 & 1.2979 & 5592.8 \\ 323.184 & 1.2867 & 3591.7 \\ 323.168 & 1.2871 & 4110.4 \\ 323.159 & 1.2903 & 4557.9 \\ 323.166 & 1.2934 & 5023.3 \\ 323.184 & 1.2998 & 6039.3 \\ 323.158 & 1.3029 & 6545.9 \\ 327.958 & 1.2476 & 1102.6 \\ 328.000 & 1.2517 & 1577.1 \\ 327.979 & 1.2564 & 2080.2 \\ 327.897 & 1.2612 & 2629.4 \\ 328.032 & 1.2644 & 3069.7 \\ 328.058 & 1.2682 & 3596.9 \\ 328.042 & 1.2690 & 4119.5 \\ 328.031 & 1.2726 & 4571.1 \\ 328.036 & 1.2759 & 5036.0 \\ 328.008 & 1.2807 & 5599.8 \\ 328.062 & 1.2828 & 6060.8 \\ 328.023 & 1.2862 & 6558.6 \\ 332.790 & 1.2262 & 1105.3 \\ 332.841 & 1.2310 & 1582.3 \\ 332.778 & 1.2362 & 2085.4 \\ 332.807 & 1.2410 & 2635.9 \\ 332.836 & 1.2450 & 3084.7 \\ 332.884 & 1.2492 & 3602.2 \\ 332.885 & 1.2506 & 4127.8 \\ 332.875 & 1.2543 & 4582.7 \\ 332.882 & 1.2579 & 5044.0 \\ 332.850 & 1.2636 & 5610.3 \\ 332.906 & 1.2654 & 6077.1 \\ 332.870 & 1.2691 & 6572.6 \\ 337.833 & 1.2083 & 1583.8 \\ 337.777 & 1.2141 & 2090.8 \\ 337.804 & 1.2197 & 2644.2 \\ 337.828 & 1.2241 & 3089.9 \\ 337.884 & 1.2288 & 3610.2 \\ 337.903 & 1.2306 & 4136.0 \\ 337.876 & 1.2349 & 4593.3 \\ 337.877 & 1.2387 & 5051.2 \\ 337.848 & 1.2472 & 5620.8 \\ 337.906 & 1.2471 & 6089.6 \\ 337.866 & 1.2510 & 6586.3 \\ 342.787 & 1.1843 & 1588.3 \\ 342.733 & 1.1910 & 2096.7 \\ 342.758 & 1.1974 & 2651.8 \\ 342.779 & 1.2024 & 3098.2 \\ 342.879 & 1.2076 & 3623.9 \\ 342.823 & 1.2105 & 4147.5 \\ & 1.2149 & 4601.9 \\ 3467 & & \\ 325 & \end{array}$




$\begin{array}{lll}342.839 & 1.2193 & 5065.9 \\ 342.808 & 1.2280 & 5632.8 \\ 342.877 & 1.2283 & 6112.2 \\ 342.824 & 1.2335 & 6600.7 \\ 347.706 & 1.1587 & 1593.0 \\ 347.635 & 1.1666 & 2103.5 \\ 347.598 & 1.1742 & 2659.2 \\ 347.696 & 1.1797 & 3110.8 \\ 347.622 & 1.1863 & 3630.5 \\ 347.781 & 1.1891 & 4155.1 \\ 347.761 & 1.1942 & 4615.9 \\ 347.760 & 1.1991 & 5081.9 \\ 347.738 & 1.2081 & 5644.6 \\ 347.796 & 1.2091 & 6128.0 \\ 347.750 & 1.2141 & 6615.5 \\ & & \\ 352.517 & 1.1405 & 2110.0 \\ 352.485 & 1.1496 & 2666.3 \\ 352.556 & 1.1558 & 3116.4 \\ 352.640 & 1.1628 & 3647.6 \\ 352.675 & 1.1669 & 4168.0 \\ 352.646 & 1.1726 & 4624.3 \\ 352.647 & 1.1781 & 5091.7 \\ 352.612 & 1.1878 & 5658.0 \\ 352.626 & 1.1896 & 6146.8 \\ 352.637 & 1.1947 & 6631.1 \\ 357.370 & 1.1122 & 2111.6 \\ 357.392 & 1.1227 & 2673.3 \\ 357.280 & 1.1307 & 3123.4 \\ 357.500 & 1.1384 & 3655.8 \\ 357.520 & 1.1437 & 4181.5 \\ 357.514 & 1.1503 & 4632.3 \\ 357.523 & 1.1562 & 5107.5 \\ 357.479 & 1.1667 & 5670.1 \\ 357.498 & 1.1691 & 6159.0 \\ 357.499 & 1.1748 & 6646.9 \\ & & \\ 362.220 & 1.0805 & 2117.4 \\ 362.239 & 1.0938 & 2683.9 \\ 362.286 & 1.1023 & 3133.6 \\ 362.312 & 1.1127 & 3669.5 \\ 362.331 & 1.1191 & 4196.0 \\ 362.307 & 1.1266 & 4648.1 \\ 362.335 & 1.1353 & 5125.8 \\ 362.289 & 1.1448 & 5684.1 \\ 362.302 & 1.1482 & 6178.7 \\ 362.314 & 1.1544 & 6663.9 \\ 367.101 & 1.0612 & 2693.5 \\ 367.106 & 1.0728 & 3143.1 \\ 367.128 & 1.0846 & 3680.5 \\ 367.154 & 1.0951 & 4198.7 \\ & & \end{array}$




$\begin{array}{lll}367.124 & 1.1038 & 4651.9 \\ 367.128 & 1.1114 & 5138.9 \\ 367.101 & 1.1219 & 5697.6 \\ 367.126 & 1.1257 & 6172.3 \\ 367.121 & 1.1332 & 6681.5 \\ & & \\ 368.942 & 1.0730 & 3671.9 \\ 369.038 & 1.0839 & 4200.9 \\ 369.063 & 1.0929 & 4647.8 \\ 368.995 & 1.1014 & 5144.2 \\ 369.175 & 1.1106 & 5709.0 \\ 369.195 & 1.1157 & 6165.5 \\ 368.579 & 1.1263 & 6685.8 \\ 372.090 & 1.0221 & 2694.7 \\ 372.366 & 1.0356 & 3147.6\end{array}$

A. 39 
Table 17b. Compressed liquid density measurements for HCFC-124 (PI units).

$\begin{array}{lrr}\text { T/F } & \text { rho/1b/ft } & \text { P/psia } \\ 35.775 & 89.346 & 57.567 \\ 35.684 & 89.584 & 71.513 \\ 35.694 & 89.608 & 87.532 \\ 35.680 & 89.704 & 152.390 \\ 35.714 & 89.558 & 228.529 \\ 35.616 & 89.683 & 298.496 \\ 35.593 & 89.771 & 358.133 \\ 35.669 & 89.886 & 440.392 \\ 35.645 & 90.005 & 520.970 \\ 35.457 & 90.120 & 573.140 \\ 35.552 & 90.202 & 653.728 \\ 35.654 & 90.297 & 717.669 \\ 35.587 & 90.395 & 793.447 \\ 36.421 & 90.398 & 873.617 \\ 35.724 & 90.552 & 922.591 \\ & & \\ 42.084 & 88.658 & 58.974 \\ 42.133 & 88.875 & 71.585 \\ 41.989 & 88.933 & 87.685 \\ 41.989 & 89.008 & 152.335 \\ 41.993 & 88.883 & 228.982 \\ 42.031 & 88.995 & 298.800 \\ 41.981 & 89.094 & 358.958 \\ 42.035 & 89.216 & 440.876 \\ 41.984 & 89.344 & 522.145 \\ 42.015 & 89.422 & 574.048 \\ 41.935 & 89.550 & 654.768 \\ 41.993 & 89.634 & 717.150 \\ 42.062 & 89.735 & 794.148 \\ 42.103 & 89.842 & 873.514 \\ 42.050 & 89.917 & 924.066 \\ & & \\ 50.914 & 87.667 & 59.556 \\ 51.038 & 87.879 & 71.645 \\ 50.864 & 87.938 & 87.877 \\ 50.848 & 88.023 & 152.453 \\ 50.873 & 87.910 & 229.536 \\ 50.866 & 88.031 & 299.216 \\ 50.826 & 88.144 & 359.692 \\ 50.894 & 88.268 & 441.510 \\ 50.874 & 88.403 & 523.323 \\ 50.912 & 88.484 & 574.959 \\ 50.841 & 88.622 & 655.982 \\ 50.856 & 88.715 & 717.670 \\ 50.882 & 88.831 & 795.372 \\ 50.911 & 88.944 & 874.495 \\ 50.895 & 89.020 & 925.995 \\ 59.709 & 86.662 & 59.843\end{array}$




$\begin{array}{llr}59.830 & 86.874 & 71.741 \\ 59.698 & 86.923 & 88.130 \\ 59.678 & 87.031 & 152.670 \\ 59.699 & 86.922 & 229.937 \\ 59.703 & 87.052 & 299.636 \\ 59.612 & 87.177 & 360.669 \\ 59.699 & 87.306 & 441.481 \\ 59.753 & 87.446 & 524.266 \\ 59.718 & 87.539 & 574.571 \\ 59.656 & 87.687 & 657.253 \\ 59.682 & 87.787 & 718.966 \\ 59.721 & 87.910 & 797.069 \\ 59.708 & 88.033 & 875.864 \\ 59.729 & 88.111 & 927.607 \\ 68.692 & 85.609 & 60.016 \\ 68.730 & 85.828 & 71.874 \\ 68.597 & 85.876 & 88.315 \\ 68.600 & 86.000 & 152.920 \\ 68.599 & 85.907 & 230.530 \\ 68.661 & 85.899 & 230.576 \\ 68.608 & 86.041 & 299.816 \\ 68.676 & 86.161 & 361.485 \\ 68.586 & 86.318 & 441.773 \\ 68.659 & 86.469 & 525.753 \\ 68.601 & 86.568 & 574.114 \\ 68.573 & 86.727 & 658.561 \\ 68.582 & 86.834 & 720.234 \\ 68.609 & 86.968 & 798.462 \\ 68.612 & 87.100 & 877.373 \\ 68.612 & 87.184 & 929.692 \\ & & \\ 77.757 & 69.052 & 62.403 \\ 77.712 & 84.742 & 72.009 \\ 77.585 & 84.786 & 88.537 \\ 77.572 & 84.941 & 153.196 \\ 77.589 & 85.001 & 300.205 \\ 77.667 & 85.129 & 362.237 \\ 77.578 & 85.300 & 442.530 \\ 77.647 & 85.463 & 526.359 \\ 77.578 & 85.571 & 575.541 \\ 77.565 & 85.740 & 660.091 \\ 77.568 & 85.855 & 721.296 \\ 77.596 & 85.999 & 799.588 \\ 77.604 & 86.141 & 878.785 \\ 77.606 & 86.233 & 931.996 \\ & & \\ 86.708 & 78.424 & 74.942 \\ 86.576 & 83.674 & 88.785 \\ 86.598 & 83.453 & 105.390 \\ 86.579 & 83.854 & 153.511 \\ 86.608 & 83.601 & 166.732 \\ 86.628 & 83.767 & 231.515 \\ 86.585 & 83.935 & 300.710\end{array}$




$\begin{array}{lcc}86.657 & 84.075 & 363.080 \\ 86.571 & 84.259 & 443.354 \\ 86.629 & 84.435 & 527.284 \\ 86.581 & 84.550 & 576.395 \\ 86.555 & 84.733 & 661.822 \\ 86.572 & 84.856 & 722.169 \\ 86.591 & 85.013 & 800.587 \\ 86.609 & 85.167 & 880.391 \\ 86.615 & 85.267 & 934.212 \\ & & \\ 95.399 & 82.551 & 88.972 \\ 95.409 & 82.775 & 153.821 \\ 95.433 & 82.864 & 231.985 \\ 95.409 & 82.864 & 301.268 \\ 95.480 & 83.012 & 363.885 \\ 95.389 & 83.216 & 444.156 \\ 95.458 & 83.406 & 528.636 \\ 95.401 & 83.528 & 577.146 \\ 95.407 & 83.725 & 663.804 \\ 95.401 & 83.858 & 723.261 \\ 95.427 & 84.030 & 803.219 \\ 95.434 & 84.193 & 882.008 \\ 95.439 & 84.302 & 935.943 \\ 104.319 & 81.568 & 158.686 \\ 104.338 & 81.766 & 226.239 \\ 104.321 & 81.973 & 299.153 \\ 104.326 & 82.183 & 376.519 \\ 104.335 & 82.354 & 441.552 \\ 104.441 & 82.532 & 517.583 \\ 104.385 & 82.525 & 593.389 \\ 104.378 & 82.694 & 658.035 \\ 104.384 & 82.852 & 724.782 \\ 104.401 & 83.091 & 810.874 \\ 104.443 & 83.399 & 872.051 \\ 104.363 & 83.367 & 945.746 \\ 113.134 & 80.385 & 159.133 \\ 113.184 & 80.600 & 227.459 \\ 113.152 & 80.830 & 300.036 \\ 113.155 & 81.062 & 378.865 \\ 113.191 & 81.244 & 442.659 \\ 113.277 & 81.444 & 519.648 \\ 113.246 & 81.451 & 595.202 \\ 113.228 & 81.635 & 659.797 \\ 113.239 & 81.813 & 727.680 \\ 113.239 & 82.066 & 812.921 \\ 113.271 & 82.188 & 873.658 \\ 113.218 & 82.361 & 947.537 \\ 121.898 & 79.162 & 159.522 \\ 121.971 & 79.396 & 228.118 \\ 121.923 & 79.656 & 300.692 \\ & & 380.331 \\ & & \end{array}$




$\begin{array}{lll}121.976 & 80.113 & 443.845 \\ 121.948 & 81.022 & 811.173 \\ 122.060 & 80.326 & 520.936 \\ 122.033 & 80.353 & 596.166 \\ 122.017 & 80.552 & 661.060 \\ 122.028 & 80.745 & 728.564 \\ 122.062 & 81.144 & 875.926 \\ 122.014 & 81.338 & 949.409 \\ 130.654 & 77.883 & 159.918 \\ 130.730 & 78.144 & 228.746 \\ 130.691 & 78.432 & 301.711 \\ 130.544 & 78.731 & 381.360 \\ 130.788 & 78.932 & 445.225 \\ 130.834 & 79.170 & 521.680 \\ 130.805 & 79.224 & 597.485 \\ 130.785 & 79.445 & 662.984 \\ 130.795 & 79.650 & 730.417 \\ 130.744 & 79.952 & 812.177 \\ 130.841 & 80.081 & 879.041 \\ 130.771 & 80.294 & 951.248 \\ 139.352 & 76.548 & 160.316 \\ 139.443 & 76.849 & 229.496 \\ 139.331 & 77.171 & 302.463 \\ 139.383 & 77.475 & 382.305 \\ 139.435 & 77.724 & 447.394 \\ 139.521 & 77.983 & 522.461 \\ 139.522 & 78.071 & 598.680 \\ 139.505 & 78.304 & 664.663 \\ 139.517 & 78.526 & 731.564 \\ 139.459 & 78.886 & 813.711 \\ 139.562 & 78.999 & 881.413 \\ 139.496 & 79.22 \varepsilon & 953.277 \\ 148.430 & 75.429 & 229.710 \\ 148.329 & 75.795 & 303.247 \\ 148.377 & 76.140 & 383.515 \\ 148.420 & 76.419 & 448.146 \\ 148.521 & 76.709 & 523.616 \\ 148.555 & 76.823 & 599.876 \\ 148.506 & 77.090 & 666.208 \\ 148.508 & 77.330 & 732.614 \\ 148.456 & 77.861 & 815.226 \\ 148.560 & 77.853 & 883.223 \\ 148.490 & 78.098 & 955.268 \\ & & \\ 157.346 & 73.933 & 230.365 \\ 157.249 & 74.351 & 304.107 \\ 157.295 & 74.749 & 384.616 \\ 157.333 & 75.063 & 449.353 \\ 157.512 & 75.385 & 525.609 \\ 157.491 & 75.570 & 601.542 \\ 157.412 & 75.842 & 667.456\end{array}$




$\begin{array}{lll}157.441 & 76.117 & 734.747 \\ 157.384 & 76.659 & 816.963 \\ 157.509 & 76.680 & 886.501 \\ 157.413 & 77.005 & 957.355 \\ & & \\ 166.201 & 72.337 & 231.049 \\ 166.073 & 72.829 & 305.093 \\ 166.007 & 73.305 & 385.691 \\ 166.183 & 73.649 & 451.180 \\ 166.049 & 74.059 & 526.561 \\ 166.335 & 74.231 & 602.643 \\ 166.300 & 74.549 & 669.486 \\ 166.298 & 74.855 & 737.067 \\ 166.258 & 75.421 & 818.685 \\ 166.363 & 75.479 & 888.788 \\ 166.281 & 75.795 & 959.493 \\ & & \\ 174.861 & 71.202 & 306.025 \\ 174.803 & 71.768 & 386.715 \\ 174.930 & 72.154 & 451.997 \\ 175.081 & 72.593 & 529.037 \\ 175.145 & 72.845 & 604.524 \\ 175.093 & 73.202 & 670.697 \\ 175.095 & 73.544 & 738.490 \\ 175.031 & 74.152 & 820.630 \\ 175.057 & 74.264 & 891.523 \\ 175.076 & 74.586 & 961.766 \\ 183.595 & 69.432 & 306.258 \\ 183.636 & 70.089 & 387.726 \\ 183.433 & 70.590 & 453.010 \\ 183.829 & 71.067 & 530.230 \\ 183.867 & 71.397 & 606.478 \\ 183.856 & 71.808 & 671.863 \\ 183.872 & 72.182 & 740.775 \\ 183.792 & 72.832 & 822.374 \\ 183.827 & 72.983 & 893.281 \\ 183.828 & 73.342 & 964.049 \\ 192.326 & 67.453 & 307.107 \\ 192.360 & 68.283 & 389.264 \\ 1.92 .445 & 68.814 & 454.492 \\ 192.492 & 69.466 & 532.216 \\ 192.526 & 69.865 & 608.574 \\ 192.483 & 70.333 & 674.151 \\ 192.533 & 70.874 & 743.427 \\ 192.449 & 71.468 & 824.413 \\ 192.473 & 71.679 & 896.146 \\ 192.496 & 72.066 & 966.523 \\ 201.111 & 66.249 & 390.658 \\ 201.121 & 66.971 & 455.875 \\ 201.161 & 67.711 & 533.805 \\ 201.208 & 68.363 & 608.965 \\ 1 & & \end{array}$




$\begin{array}{lll}201.153 & 68.906 & 674.695 \\ 201.160 & 69.384 & 745.329 \\ 201.111 & 70.037 & 826.363 \\ 201.157 & 70.273 & 895.214 \\ 201.148 & 70.742 & 969.063 \\ & & \\ 204.426 & 66.984 & 532.559 \\ 204.598 & 67.663 & 609.294 \\ 204.643 & 68.229 & 674.104 \\ 204.521 & 68.759 & 746.106 \\ 204.846 & 69.334 & 828.026 \\ 204.881 & 69.651 & 894.235 \\ 203.773 & 70.313 & 969.697 \\ 210.092 & 63.809 & 390.833 \\ 210.588 & 64.650 & 456.514\end{array}$


Table 18. Ebulliometric vapor pressure measurements for HCFC-124 (R-124).

Vapor Pressure of 2, Chloro - 1,1,1,2 - Tetrafluoroethane (R124)

\begin{tabular}{ccccc}
$\begin{array}{c}\text { Point } \\
\text { Number }\end{array}$ & $\begin{array}{c}\mathrm{T} \\
(\mathrm{K})\end{array}$ & $\begin{array}{c}\mathrm{P} \\
(\mathrm{kPa})\end{array}$ & $\begin{array}{c}\mathrm{T} \\
(\mathrm{F})\end{array}$ & $\begin{array}{c}\mathrm{P} \\
(\mathrm{ps} \text { ia })\end{array}$ \\
\hline 1 & 221.808 & 13.6130 & -60.417 & 1.974 \\
2 & 223.005 & 14.6632 & -58.261 & 2.127 \\
3 & 222.957 & 14.6194 & -58.348 & 2.120 \\
4 & 224.068 & 15.6360 & -56.348 & 2.268 \\
5 & 225.082 & 16.6252 & -54.522 & 2.411 \\
6 & 226.970 & 18.5812 & -51.124 & 2.695 \\
7 & 228.600 & 20.4246 & -48.190 & 2.962 \\
8 & 230.427 & 22.6687 & -44.902 & 3.288 \\
9 & 231.986 & 24.7343 & -42.095 & 3.587 \\
10 & 233.583 & 27.0102 & -39.220 & 3.918 \\
11 & 235.514 & 29.9858 & -35.745 & 4.349 \\
12 & 237.217 & 32.8220 & -32.679 & 4.760 \\
13 & 239.027 & 36.0745 & -29.422 & 5.232 \\
14 & 241.623 & 41.1778 & -24.748 & 5.972 \\
15 & 243.917 & 46.1688 & -20.620 & 6.696 \\
16 & 243.488 & 45.2053 & -21.392 & 6.556 \\
17 & 245.627 & 50.1826 & -17.542 & 7.278 \\
18 & 247.696 & 55.4309 & -13.818 & 8.040 \\
19 & 249.587 & 60.5849 & -10.414 & 8.787 \\
20 & 251.187 & 65.2398 & -7.534 & 9.462 \\
21 & 252.720 & 69.9698 & -4.774 & 10.148 \\
22 & 254.415 & 75.5098 & -1.724 & 10.952 \\
23 & 255.598 & 79.5825 & 0.406 & 11.543 \\
24 & 257.300 & 85.7325 & 3.470 & 12.434 \\
25 & 258.405 & 89.9264 & 5.460 & 13.043 \\
26 & 259.892 & 95.8242 & 8.136 & 13.898 \\
27 & 260.959 & 100.2267 & 10.056 & 14.537 \\
28 & 263.330 & 110.5957 & 14.325 & 16.041 \\
29 & 265.432 & 120.4906 & 18.108 & 17.476 \\
30 & 267.404 & 130.3797 & 21.658 & 18.910 \\
31 & 269.214 & 140.0035 & 24.915 & 20.306 \\
32 & 271.077 & 150.4806 & 28.269 & 21.825 \\
33 & 272.883 & 161.1857 & 31.519 & 23.378 \\
34 & 274.270 & 169.8534 & 34.017 & 24.635 \\
35 & 275.821 & 179.9463 & 36.808 & 26.099 \\
36 & 277.550 & 191.6724 & 39.920 & 27.800 \\
37 & 279.074 & 202.4828 & 42.663 & 29.368 \\
38 & 280.273 & 211.3804 & 44.821 & 30.658 \\
39 & 282.200 & 226.2593 & 48.290 & 32.816 \\
40 & 283.872 & 239.7535 & 51.299 & 34.773 \\
41 & 286.098 & 258.8288 & 55.307 & 37.540
\end{tabular}


Table 19a. Fit of the HCFC-124 (R-124) vapor pressure measurements to an Antoine equation (SI units). The percent deviation is $100 \times\left(P-P_{\text {calc }}\right) / P_{\text {calc }}$.

\begin{tabular}{|c|c|c|c|c|}
\hline \multirow{2}{*}{$\begin{array}{c}\text { Pcalc = } \\
\text { Point } \\
\text { Number }\end{array}$} & \multicolumn{2}{|c|}{$\exp (A+(B /(T+C)))$} & \multicolumn{2}{|c|}{$\begin{array}{l}A=13.93900 \\
B=-2073.45 \\
C=-38.76133\end{array}$} \\
\hline & $\begin{array}{l}T \\
(K)\end{array}$ & $\underset{(\mathrm{kPa})}{\mathrm{P}}$ & $\begin{array}{r}\mathrm{Pcalc} \\
(\mathrm{kPa})\end{array}$ & $\begin{array}{c}\text { Deviat } \\
\%\end{array}$ \\
\hline 1 & 221.808 & 13.613 & 13.620 & -0.050 \\
\hline 2 & 223.005 & 14.663 & 14.660 & 0.019 \\
\hline 3 & 222.957 & 14.619 & 14.617 & 0.016 \\
\hline 4 & 224.068 & 15.636 & 15.638 & -0.010 \\
\hline 5 & 225.082 & 16.625 & 16.620 & 0.031 \\
\hline 6 & 226.970 & 18.581 & 18.583 & -0.007 \\
\hline 7 & 228.600 & 20.425 & 20.426 & -0.009 \\
\hline 8 & 230.427 & 22.669 & 22.668 & 0.005 \\
\hline 9 & 231.986 & 24.734 & 24.736 & -0.005 \\
\hline 10 & 233.583 & 27.010 & 27.010 & 0.002 \\
\hline 11 & 235.514 & 29.986 & 29.983 & 0.010 \\
\hline 12 & 237.217 & 32.822 & 32.822 & 0.001 \\
\hline 13 & 239.027 & 36.075 & 36.071 & 0.011 \\
\hline 14 & 241.623 & 41.178 & 41.182 & -0.011 \\
\hline 15 & 243.917 & 46.169 & 46.167 & 0.004 \\
\hline 16 & 243.488 & 45.205 & 45.199 & 0.013 \\
\hline 17 & 245.627 & 50.183 & 50.189 & -0.013 \\
\hline 18 & 247.696 & 55.431 & 55.427 & 0.007 \\
\hline 19 & 249.587 & 60.585 & 60.588 & -0.004 \\
\hline 20 & 251.187 & 65.240 & 65.245 & -0.008 \\
\hline 21 & 252.720 & 69.970 & 69.972 & -0.003 \\
\hline 22 & 254.415 & 75.510 & 75.510 & 0.000 \\
\hline 23 & 255.598 & 79.582 & 79.577 & 0.007 \\
\hline 24 & 257.300 & 85.732 & 85.731 & 0.002 \\
\hline 25 & 258.405 & 89.926 & 89.922 & 0.004 \\
\hline 26 & 259.892 & 95.824 & 95.814 & 0.010 \\
\hline 27 & 260.959 & 100.227 & 100.227 & -0.000 \\
\hline 28 & 263.330 & 110.596 & 110.607 & -0.010 \\
\hline 29 & 265.432 & 120.491 & 120.494 & -0.003 \\
\hline 30 & 267.404 & 130.380 & 130.385 & -0.004 \\
\hline 31 & 269.214 & 140.004 & 140.008 & -0.003 \\
\hline 32 & 271.077 & 150.481 & 150.486 & -0.004 \\
\hline 33 & 272.883 & 161.186 & 161.209 & -0.015 \\
\hline 34 & 274.270 & 169.853 & 169.843 & 0.006 \\
\hline 35 & 275.821 & 179.946 & 179.913 & 0.019 \\
\hline 36 & 277.550 & 191.672 & 191.673 & -0.000 \\
\hline 37 & 279.074 & 202.483 & 202.523 & -0.020 \\
\hline 38 & 280.273 & 211.380 & 211.388 & -0.003 \\
\hline 39 & 282.200 & 226.259 & 226.252 & 0.003 \\
\hline 40 & 283.872 & 239.753 & 239.786 & -0.014 \\
\hline 41 & 286.098 & 258.829 & 258.760 & 0.027 \\
\hline
\end{tabular}


Table 19b. Fit of the HCFC-124 (R-124) vapor pressure measurements to an Antoine equation (PI units). The percent deviation is $100 \times\left(P-P_{\text {calc }}\right) / P_{\text {calc }}$.

Antoine equation - R124 (eng. units)

$\begin{array}{ll}\text { Pcalc }=\exp (A+(B /(T+C))) & A=12.00824 \\ & B=-3732.21 \\ C=389.89961\end{array}$

\begin{tabular}{|c|c|c|c|c|}
\hline $\begin{array}{l}\text { Point } \\
\text { Number }\end{array}$ & $\stackrel{T}{(F)}$ & $\frac{\mathrm{P}}{(p s i a)}$ & $\begin{array}{l}\text { Pcalc } \\
\text { (psia) }\end{array}$ & $\begin{array}{c}\text { Deviat } \\
\%\end{array}$ \\
\hline 1 & -60.417 & 1.974 & 1.975 & -0.050 \\
\hline 2 & -58.261 & 2.127 & 2.126 & 0.020 \\
\hline 3 & -58.348 & 2.120 & 2.120 & 0.016 \\
\hline 4 & -56.348 & 2.268 & 2.268 & -0.010 \\
\hline 5 & -54.522 & 2.411 & 2.411 & 0.032 \\
\hline 6 & -51.124 & 2.695 & 2.695 & -0.007 \\
\hline 7 & $-48 \cdot 190$ & 2.962 & 2.963 & -0.009 \\
\hline 8 & -44.902 & 3.288 & 3.288 & 0.005 \\
\hline 9 & -42.095 & 3.587 & 3.588 & -0.005 \\
\hline 10 & -39.220 & 3.918 & 3.917 & 0.002 \\
\hline 11 & -35.745 & 4.349 & 4.349 & 0.010 \\
\hline 12 & -32.679 & 4.760 & 4.760 & 0.001 \\
\hline 13 & -29.422 & 5.232 & 5.232 & 0.011 \\
\hline 14 & -24.748 & 5.972 & 5.973 & -0.010 \\
\hline 15 & -20.620 & 6.696 & 6.696 & 0.004 \\
\hline 16 & -21.392 & 6.556 & 6.556 & 0.014 \\
\hline 17 & -17.542 & 7.278 & 7.279 & -0.013 \\
\hline 18 & -13.818 & 8.040 & 8.039 & 0.007 \\
\hline 19 & -10.414 & 8.787 & 8.787 & -0.004 \\
\hline 20 & -7.534 & 9.462 & 9.463 & -0.008 \\
\hline 21 & -4.774 & 10.148 & 10.149 & -0.003 \\
\hline 22 & -1.724 & 10.952 & 10.952 & 0.000 \\
\hline 23 & 0.406 & 11.543 & 11.542 & 0.007 \\
\hline 24 & 3.470 & 12.434 & 12.434 & 0.002 \\
\hline 25 & 5.460 & 13.043 & 13.042 & 0.005 \\
\hline 26 & 8.136 & 13.898 & 13.897 & 0.011 \\
\hline 27 & 10.056 & 14.537 & 14.537 & 0.000 \\
\hline 28 & 14.325 & 16.041 & 16.042 & -0.010 \\
\hline 29 & 18.108 & 17.476 & 17.476 & -0.003 \\
\hline 30 & 21.658 & 18.910 & 18.911 & -0.004 \\
\hline 31 & 24.915 & 20.306 & 20.306 & -0.003 \\
\hline 32 & 28.269 & 21.825 & 21.826 & -0.003 \\
\hline 33 & 31.519 & 23.378 & 23.381 & -0.014 \\
\hline 34 & 34.017 & 24.635 & 24.634 & 0.007 \\
\hline 35 & 36.808 & 26.099 & 26.094 & 0.019 \\
\hline 36 & 39.920 & 27.800 & 27.800 & -0.000 \\
\hline 37 & 42.663 & 29.368 & 29.374 & -0.020 \\
\hline 38 & 44.821 & 30.658 & 30.659 & -0.003 \\
\hline 39 & 48.290 & 32.816 & 32.815 & 0.004 \\
\hline 40 & 51.299 & 34.773 & 34.778 & -0.013 \\
\hline 41 & 55.307 & 37.540 & 37.530 & 0.027 \\
\hline
\end{tabular}


Table 20a. Compressed liquid density measurements for HFC-125 (SI units).

\begin{tabular}{|c|c|c|}
\hline $\mathrm{T} / \mathrm{K}$ & $\mathrm{P} / \mathrm{kPa}$ & Tho $8 / c c$ \\
\hline $\begin{array}{l}275.559 \\
275.553 \\
275.412 \\
275.407 \\
275.368 \\
275.374\end{array}$ & $\begin{array}{l}1590.1 \\
2019.8 \\
3006.5 \\
3996.3 \\
4958.9 \\
6213.0\end{array}$ & $\begin{array}{l}1.3160 \\
1.3194 \\
1.3272 \\
1.3341 \\
1.3406 \\
1.3484\end{array}$ \\
\hline $\begin{array}{l}283.966 \\
283.978 \\
283.985 \\
283.997 \\
284.000 \\
284.003\end{array}$ & $\begin{array}{l}1607.4 \\
2019.9 \\
3024.5 \\
4057.0 \\
5070.0 \\
6238.5\end{array}$ & $\begin{array}{l}1.2745 \\
1.2783 \\
1.2873 \\
1.2958 \\
1.3037 \\
1.3122\end{array}$ \\
\hline $\begin{array}{l}293.477 \\
293.479 \\
293.482 \\
293.483 \\
293.484 \\
293.482\end{array}$ & $\begin{array}{l}1586.8 \\
2054.7 \\
3005.2 \\
4035.0 \\
5073.5 \\
6266.3\end{array}$ & $\begin{array}{l}1.2217 \\
1.2275 \\
1.2384 \\
1.2493 \\
1.2593 \\
1.2 \% 00\end{array}$ \\
\hline $\begin{array}{l}303.313 \\
303.323 \\
303.326 \\
303.331 \\
303.330 \\
303.353\end{array}$ & $\begin{array}{l}1616.2 \\
2026.6 \\
3053.0 \\
4021.6 \\
5049.6 \\
6283.0\end{array}$ & $\begin{array}{l}1.1586 \\
1.1659 \\
1.1824 \\
1.1960 \\
1.2089 \\
1.2226\end{array}$ \\
\hline $\begin{array}{l}313.940 \\
313.934 \\
313.932 \\
313.930 \\
313.935\end{array}$ & $\begin{array}{l}2496.4 \\
3057.2 \\
4044.2 \\
5001.2 \\
6263.2\end{array}$ & $\begin{array}{l}1.0951 \\
1.1094 \\
1.1302 \\
1.1471 \\
1.1663\end{array}$ \\
\hline $\begin{array}{l}323.873 \\
323.872 \\
323.873 \\
323.877 \\
323.872\end{array}$ & $\begin{array}{l}2811.2 \\
3039.2 \\
4016.5 \\
5027.3 \\
6212.2\end{array}$ & $\begin{array}{l}1.0067 \\
1.0178 \\
1.0534 \\
1.0807 \\
1.1059\end{array}$ \\
\hline $\begin{array}{l}333.921 \\
333.919 \\
333.909 \\
333.916\end{array}$ & $\begin{array}{l}3389.5 \\
4088.8 \\
5063.8 \\
6184.0\end{array}$ & $\begin{array}{r}.8857 \\
.9498 \\
.9977 \\
1.0345\end{array}$ \\
\hline $\begin{array}{l}339.059 \\
339.072 \\
339.062 \\
339.051\end{array}$ & $\begin{array}{l}3816.3 \\
4067.3 \\
4547.7 \\
5069.3\end{array}$ & $\begin{array}{l}.8179 \\
.8618 \\
.9098 \\
.9440\end{array}$ \\
\hline
\end{tabular}




$\begin{array}{lll}339.057 & 5535.5 & .9671 \\ 339.062 & 6168.6 & .9922 \\ & & \\ 340.499 & 3556.5 & .3437 \\ 340.504 & 3645.2 & .4761 \\ 340.494 & 3689.5 & .6451 \\ 340.499 & 3856.1 & .7717 \\ 340.509 & 4078.5 & .8272 \\ 340.520 & 4172.2 & .8429 \\ 340.605 & 4403.9 & .8710 \\ 340.598 & 4646.5 & .8945 \\ 340.594 & 4865.4 & .9118 \\ 340.571 & 5053.0 & .9246 \\ 340.576 & 5235.1 & .9355 \\ 340.603 & 5467.7 & .9476 \\ 340.598 & 5672.5 & .9576 \\ 340.577 & 5879.3 & .9671 \\ 340.499 & 6328.8 & .9855 \\ & & \\ 343.332 & 3542.8 & .2906 \\ 343.338 & 3645.5 & .3194 \\ 343.363 & 3747.6 & .3633 \\ 343.361 & 3851.0 & .4748 \\ 343.358 & 3950.3 & .6392 \\ 343.347 & 4081.3 & .7250 \\ 343.371 & 4345.8 & .7993 \\ 343.346 & 4570.0 & .8369 \\ 343.345 & 5070.8 & .8883 \\ 343.347 & 5551.8 & .9212 \\ 343.358 & 6083.4 & .9486 \\ & & \\ 348.423 & 3551.5 & .2584 \\ 348.409 & 3638.6 & .2734 \\ 348.428 & 3740.4 & .2933 \\ 348.412 & 3854.5 & .3198 \\ 348.431 & 3946.2 & .3445 \\ 348.448 & 4041.8 & .3778 \\ 348.444 & 4152.0 & .4362 \\ 348.453 & 4251.6 & .5078 \\ 348.448 & 4348.2 & .5809 \\ 348.513 & 4581.2 & .6915 \\ 348.441 & 4863.6 & .7662 \\ 348.520 & 5056.5 & .7972 \\ 348.439 & 5302.9 & .8300 \\ 348.517 & 5542.6 & .8525 \\ 348.444 & 5845.1 & .8778 \\ 348.516 & 6150.1 & .8373 \\ 353.548 & 3782.0 & .2694 \\ 353.570 & 3905.1 & .2897 \\ 353.565 & 3999.3 & .3070 \\ 353.581 & 4105.9 & .3287 \\ 353.602 & 4208.7 & .3524 \\ 353.592 & 4307.0 & .3792 \\ & & \\ 345\end{array}$




$\begin{array}{lll}353.549 & 4411.5 & .4138 \\ 353.262 & 4503.4 & .4579 \\ 353.262 & 4599.4 & .5020 \\ 353.268 & 4685.0 & .5425 \\ 353.253 & 4800.1 & .5933 \\ 353.255 & 4903.5 & .6316 \\ 353.265 & 5008.3 & .6644 \\ 353.278 & 5075.3 & .6829 \\ 353.188 & 5328.9 & .7399 \\ 353.282 & 5548.4 & .7735 \\ 353.164 & 5849.9 & .8116 \\ 353.283 & 6161.8 & .8389 \\ & & \\ 358.631 & 4129.3 & .2973 \\ 358.636 & 4193.6 & .3078 \\ 358.652 & 4301.3 & .3263 \\ 358.628 & 4379.2 & .3412 \\ 358.651 & 4505.2 & .3673 \\ 358.643 & 4599.5 & .3890 \\ 358.643 & 4599.5 & .3890 \\ 358.630 & 4700.7 & .4150 \\ 358.642 & 4810.0 & .4454 \\ 358.646 & 4904.2 & .4743 \\ 358.652 & 5005.6 & .5060 \\ 358.661 & 5105.2 & .5381 \\ 358.669 & 5208.1 & .5700 \\ 358.641 & 5284.8 & .5927 \\ 358.675 & 5393.9 & .6211 \\ 358.695 & 5603.8 & .6692 \\ 358.698 & 5801.0 & .7062 \\ 358.675 & 6212.2 & .7647 \\ & & \\ 363.827 & 4206.3 & .2826 \\ 363.806 & 4296.1 & .2950 \\ 363.813 & 4387.6 & .3080 \\ 363.828 & 4503.4 & .3255 \\ 363.839 & 4604.7 & .3421 \\ 363.903 & 4703.8 & .3590 \\ 363.254 & 4799.1 & .3823 \\ 363.255 & 4898.2 & .4029 \\ 363.268 & 4998.8 & .4248 \\ 363.285 & 5092.2 & .4463 \\ 363.414 & 5196.3 & .4689 \\ 363.675 & 5310.8 & .4939 \\ 363.567 & 5415.6 & .5210 \\ 363.606 & 5498.2 & .5407 \\ 363.670 & 5611.2 & .5662 \\ 363.661 & 5798.9 & .6080 \\ 363.655 & 5911.1 & .6307 \\ 363.680 & 6000.4 & .6471 \\ 363.678 & 6105.5 & .6655 \\ 363.675 & 6254.7 & .6893 \\ 369.108 & 4411.1 & .2870\end{array}$




$\begin{array}{lll}369.134 & 4509.1 & .2991 \\ 369.134 & 4600.3 & .3111 \\ 368.406 & 5410.4 & .4495 \\ 368.468 & 5505.8 & .4677 \\ 368.565 & 5604.2 & .4862 \\ 368.483 & 5705.9 & .5089 \\ 368.304 & 5804.5 & .5302 \\ 368.436 & 5900.0 & .5477 \\ 368.525 & 5966.2 & .5584 \\ 368.525 & 5966.2 & .5584 \\ 368.578 & 6086.1 & .5800 \\ 368.586 & 6226.9 & .6048 \\ 369.188 & 4704.1 & .3249 \\ 369.173 & 4798.6 & .3384 \\ 369.192 & 4921.1 & .3568 \\ 369.292 & 5003.3 & .3687 \\ 369.292 & 5003.3 & .3687 \\ 369.347 & 5096.6 & .3832 \\ 369.361 & 5194.2 & .3994 \\ 369.449 & 5320.5 & .4206\end{array}$


Table 20b. Compressed 11quid density measurements for HFC-125 (PI units).

$\begin{array}{llc}\text { T/F } & \text { P/ps1a } & \text { rho/1b/ft } \\ & & \\ 36.336 & 230.62 & 82.155 \\ 36.325 & 292.95 & 82.367 \\ 36.072 & 436.06 & 82.854 \\ 36.063 & 579.61 & 83.285 \\ 35.992 & 719.23 & 83.691 \\ 36.003 & 901.12 & 84.178 \\ & & \\ 51.469 & 233.13 & 79.564 \\ 51.490 & 292.96 & 79.802 \\ 51.503 & 438.67 & 80.364 \\ 51.525 & 588.42 & 80.894 \\ 51.530 & 735.34 & 81.387 \\ 51.535 & 904.82 & 81.918 \\ & & \\ 68.589 & 230.15 & 76.268 \\ 68.592 & 298.01 & 76.630 \\ 68.598 & 435.87 & 77.311 \\ 68.599 & 585.23 & 77.991 \\ 68.601 & 735.85 & 78.616 \\ 68.598 & 908.85 & 79.284 \\ & & \\ 86.293 & 234.41 & 72.329 \\ 86.311 & 293.93 & 72.785 \\ 86.317 & 442.80 & 73.815 \\ 86.326 & 583.28 & 74.664 \\ 86.324 & 732.38 & 75.469 \\ 86.365 & 911.27 & 76.324 \\ & & \\ 105.422 & 362.07 & 68.365 \\ 105.411 & 443.41 & 69.258 \\ 105.408 & 586.56 & 70.556 \\ 105.404 & 725.36 & 71.611 \\ 105.413 & 908.40 & 72.810 \\ & & \\ 123.301 & 407.73 & 62.846 \\ 123.300 & 440.80 & 63.539 \\ 123.301 & 582.54 & 65.762 \\ 123.309 & 729.15 & 67.466 \\ 123.300 & 901.00 & 69.039 \\ & & \\ 141.388 & 491.61 & 55.292 \\ 141.384 & 593.03 & 59.294 \\ 141.366 & 734.44 & 62.284 \\ 141.379 & 896.91 & 64.582 \\ 150.636 & 553.51 & 51.060 \\ 150.660 & 589.91 & 53.800 \\ 150.642 & 659.59 & 56.797 \\ 150.622 & 735.24 & 58.932\end{array}$




$\begin{array}{lll}150.633 & 802.86 & 60.374 \\ 150.642 & 894.68 & 61.941 \\ 153.228 & 515.83 & 21.456 \\ 153.237 & 528.69 & 29.722 \\ 153.219 & 535.12 & 40.272 \\ 153.228 & 559.28 & 48.176 \\ 153.246 & 591.54 & 51.640 \\ 153.266 & 605.13 & 52.621 \\ 153.419 & 638.73 & 54.375 \\ 153.406 & 673.92 & 55.842 \\ 153.399 & 705.67 & 56.922 \\ 153.358 & 732.88 & 57.721 \\ 153.367 & 759.29 & 58.401 \\ 153.415 & 793.02 & 59.157 \\ 153.406 & 822.73 & 59.781 \\ 153.369 & 852.72 & 60.374 \\ 153.228 & 917.91 & 61.523 \\ 158.328 & 513.84 & 18.142 \\ 158.338 & 528.74 & 19.940 \\ 158.383 & 543.54 & 22.680 \\ 158.380 & 558.54 & 29.641 \\ 158.374 & 572.94 & 39.904 \\ 158.355 & 591.94 & 45.260 \\ 158.398 & 630.30 & 49.899 \\ 158.353 & 662.82 & 52.246 \\ 158.351 & 735.46 & 55.455 \\ 158.355 & 805.22 & 57.509 \\ 158.374 & 882.32 & 59.219 \\ 167.491 & 515.10 & 16.131 \\ 167.466 & 527.73 & 17.068 \\ 167.500 & 542.50 & 18.310 \\ 167.472 & 559.05 & 19.964 \\ 167.506 & 572.35 & 21.506 \\ 167.536 & 586.21 & 23.585 \\ 167.529 & 602.20 & 27.231 \\ 167.545 & 616.64 & 31.701 \\ 167.536 & 630.65 & 36.264 \\ 167.653 & 664.45 & 43.169 \\ 167.524 & 705.41 & 47.832 \\ 167.666 & 733.38 & 49.768 \\ 167.520 & 769.12 & 51.815 \\ 167.661 & 803.89 & 53.220 \\ 167.529 & 847.76 & 54.799 \\ 167.659 & 892.00 & 56.017 \\ & & \\ 176.716 & 548.53 & 16.818 \\ 176.756 & 566.39 & 18.085 \\ 176.747 & 580.05 & 19.165 \\ 176.776 & 595.51 & 20.520 \\ 176.814 & 610.42 & 22.000 \\ 176.796 & 624.68 & 23.673\end{array}$




$\begin{array}{lll}176.718 & 639.83 & 25.833 \\ 176.202 & 653.16 & 28.586 \\ 176.202 & 667.09 & 31.339 \\ 176.212 & 679.50 & 33.867 \\ 176.185 & 696.20 & 37.039 \\ 176.189 & 711.19 & 39.430 \\ 176.207 & 726.39 & 41.477 \\ 176.230 & 736.11 & 42.632 \\ 176.068 & 772.89 & 46.190 \\ 176.238 & 804.73 & 48.288 \\ 176.025 & 848.46 & 50.667 \\ 176.239 & 893.69 & 52.371 \\ & & \\ 185.866 & 598.90 & 18.560 \\ 185.875 & 608.23 & 19.215 \\ 185.904 & 623.85 & 20.370 \\ 185.860 & 635.15 & 21.300 \\ 185.902 & 653.42 & 22.930 \\ 185.887 & 667.10 & 24.284 \\ 185.887 & 667.10 & 24.284 \\ 185.864 & 681.78 & 25.908 \\ 185.886 & 697.63 & 27.805 \\ 185.893 & 711.29 & 29.610 \\ 185.904 & 726.00 & 31.589 \\ 185.920 & 740.45 & 33.592 \\ 185.934 & 755.37 & 35.584 \\ 185.884 & 766.50 & 37.001 \\ 185.945 & 782.32 & 38.774 \\ 185.981 & 812.76 & 41.777 \\ 185.986 & 841.36 & 44.087 \\ 185.945 & 901.00 & 47.739 \\ & & \\ 195.219 & 610.07 & 17.642 \\ 195.181 & 623.10 & 18.416 \\ 195.193 & 636.37 & 19.228 \\ 195.220 & 653.16 & 20.320 \\ 195.240 & 667.86 & 21.357 \\ 195.355 & 682.23 & 22.412 \\ 194.187 & 696.05 & 23.866 \\ 194.189 & 710.42 & 25.152 \\ 194.212 & 725.01 & 26.519 \\ 194.243 & 738.56 & 27.862 \\ 194.475 & 753.66 & 29.272 \\ 194.945 & 770.27 & 30.5 .33 \\ 194.751 & 785.47 & 32.525 \\ 194.821 & 797.45 & 33.755 \\ 194.936 & 813.84 & 35.347 \\ 194.920 & 841.06 & 37.956 \\ 194.909 & 857.33 & 39.373 \\ 194.954 & 870.28 & 40.397 \\ 194.950 & 885.53 & 41.546 \\ 194.945 & 907.17 & 43.032 \\ & & \\ 204.724 & 639.78 & 17.917\end{array}$




$\begin{array}{lll}204.771 & 653.99 & 18.672 \\ 204.771 & 667.22 & 19.421 \\ 203.461 & 784.71 & 28.061 \\ 203.572 & 798.55 & 29.198 \\ 203.747 & 812.82 & 30.352 \\ 203.599 & 827.57 & 31.770 \\ 203.277 & 841.87 & 33.099 \\ 203.515 & 855.72 & 34.192 \\ 203.675 & 865.32 & 34.860 \\ 203.675 & 865.32 & 34.860 \\ 203.770 & 882.71 & 36.208 \\ 203.785 & 903.14 & 37.756 \\ 204.868 & 682.27 & 20.283 \\ 204.841 & 695.98 & 21.126 \\ 204.876 & 713.75 & 22.274 \\ 205.056 & 725.67 & 23.017 \\ 205.056 & 725.67 & 23.017 \\ 205.155 & 739.20 & 23.922 \\ 205.180 & 753.36 & 24.934 \\ 205.338 & 771.67 & 26.257\end{array}$


Table 21(a). I ochoric PVT Measurements for HCFC-125 in SI units.

\begin{tabular}{|c|c|c|}
\hline $\begin{array}{l}\text { Temperature } \\
\text { Kelvin }\end{array}$ & $\begin{array}{l}\text { Pressure } \\
\text { MPa }\end{array}$ & $\begin{array}{l}\text { Density } \\
\mathrm{mol} / \mathrm{dm}^{3}\end{array}$ \\
\hline $\begin{array}{l}174.001 \\
176.000 \\
178.000 \\
180.001 \\
182.000 \\
184.000 \\
186.001 \\
188.001 \\
190.002 \\
192.000 \\
194.001\end{array}$ & $\begin{array}{r}1.572130 \\
3.186809 \\
6.011646 \\
9.259311 \\
12.535940 \\
15.826000 \\
19.108860 \\
22.384900 \\
25.655180 \\
28.904070 \\
32.147290\end{array}$ & $\begin{array}{l}14.06486 \\
14.05461 \\
14.02624 \\
14.02117 \\
14.01707 \\
14.01339 \\
14.00994 \\
14.00667 \\
14.00352 \\
14.00046 \\
13.99746\end{array}$ \\
\hline $\begin{array}{l}185.999 \\
188.000 \\
190.000 \\
192.001 \\
194.000 \\
196.000 \\
198.000 \\
200.000 \\
202.000 \\
204.001 \\
205.999 \\
208.000\end{array}$ & $\begin{array}{r}2.588665 \\
3.931049 \\
6.785781 \\
9.737449 \\
12.702930 \\
15.672670 \\
18.642740 \\
21.605980 \\
24.559160 \\
27.505050 \\
30.429050 \\
33.352820\end{array}$ & $\begin{array}{l}13.76481 \\
13.73541 \\
13.72883 \\
13.72439 \\
13.72062 \\
13.71718 \\
13.71395 \\
13.71085 \\
13.70785 \\
13.70493 \\
13.70208 \\
13.69928\end{array}$ \\
\hline $\begin{array}{l}202.000 \\
204.000 \\
206.001 \\
208.001 \\
210.000 \\
212.000 \\
214.000 \\
216.000 \\
217.999 \\
219.999 \\
221.999 \\
223.998 \\
226.000 \\
228.000 \\
230.001\end{array}$ & $\begin{array}{r}1.547702 \\
3.168674 \\
5.107316 \\
7.612479 \\
10.149190 \\
12.695070 \\
15.243050 \\
17.786270 \\
20.321700 \\
22.850930 \\
25.373530 \\
27.893440 \\
30.405660 \\
32.905790 \\
35.396040\end{array}$ & $\begin{array}{l}13.31333 \\
13.30339 \\
13.27617 \\
13.27139 \\
13.26759 \\
13.26421 \\
13.26107 \\
13.25810 \\
13.25524 \\
13.25247 \\
13.24975 \\
13.24709 \\
13.24446 \\
13.24188 \\
13.23931\end{array}$ \\
\hline $\begin{array}{l}220.000 \\
223.999 \\
228.000 \\
232.000 \\
236.000 \\
240.000 \\
244.001 \\
248.000\end{array}$ & $\begin{array}{r}3.584159 \\
7.887842 \\
12.303330 \\
16.710760 \\
21.108060 \\
25.487380 \\
29.846050 \\
34.172920\end{array}$ & $\begin{array}{l}12.85620 \\
12.84580 \\
12.83926 \\
12.83358 \\
12.82829 \\
12.82321 \\
12.81830 \\
12.81348\end{array}$ \\
\hline
\end{tabular}


Table 21(a) (continued). Isochoric PVT Measurements for HCFC-125 in SI units.

$\begin{array}{ccc}\begin{array}{c}\text { Temperature } \\ \text { Ke1vin }\end{array} & \begin{array}{c}\text { Pressure } \\ \text { MPa }\end{array} & \begin{array}{l}\text { Density } \\ \text { mol/dm }\end{array} \\ 244.001 & & \\ 248.001 & 3.150607 & 12.13794 \\ 252.001 & 6.081187 & 12.10767 \\ 256.002 & 9.523219 & 12.10132 \\ 260.000 & 12.978420 & 12.09596 \\ 263.998 & 16.431190 & 12.09103 \\ 267.998 & 19.871730 & 12.08633 \\ 271.998 & 23.300320 & 12.08178 \\ 275.999 & 30.121690 & 12.07733 \\ 279.999 & 33.511270 & 12.07296 \\ & & 12.06868 \\ 276.001 & 2.664308 & 11.01460 \\ 279.999 & 4.587385 & 10.98287 \\ 284.001 & 6.952455 & 10.97676 \\ 288.001 & 9.330561 & 10.97185 \\ 292.001 & 11.714370 & 10.96739 \\ 296.001 & 14.099870 & 10.96318 \\ 300.002 & 16.483800 & 10.95911 \\ 304.002 & 18.866340 & 10.95515 \\ 308.002 & 21.235980 & 10.95125 \\ 311.999 & 23.607610 & 10.94741 \\ 316.001 & 25.974380 & 10.94359 \\ 320.001 & 28.340111 & 10.93980 \\ 323.998 & 30.694960 & 10.93605 \\ 327.999 & 33.033720 & 10.93242 \\ 332.001 & 35.372100 & 10.92885 \\ 310.000 & 2.472744 & 9.38636 \\ 313.999 & 3.683955 & 9.35658 \\ 318.000 & 5.050635 & 9.35113 \\ 322.001 & 6.431671 & 9.34684 \\ 326.001 & 7.823143 & 9.34301 \\ 330.000 & 9.220093 & 9.33942 \\ 334.002 & 10.622020 & 9.33598 \\ 338.000 & 12.025280 & 9.33293 \\ 342.001 & 13.435840 & 9.32983 \\ 350.002 & 16.261530 & 9.32336 \\ 358.000 & 19.091220 & 9.31698 \\ 366.000 & 21.921170 & 9.31066 \\ 374.001 & 24.747190 & 9.30436 \\ 382.000 & 27.567680 & 9.29806 \\ 389.998 & 30.378470 & 9.29176 \\ 398.001 & 33.180550 & 9.28542\end{array}$


Table 21(b). Isochoric PVT Measurements for HCFC-125 in PI units.

\begin{tabular}{|c|c|c|}
\hline $\begin{array}{l}\text { Temperature } \\
\text { Fahrenheit }\end{array}$ & $\begin{array}{l}\text { Pressure } \\
\text { psia }\end{array}$ & $\begin{array}{c}\text { Density } \\
1 \mathrm{~b} \text {-mass } / \mathrm{ft}^{3}\end{array}$ \\
\hline $\begin{array}{l}-146.47 \\
-142.87 \\
-139.27 \\
-135.67 \\
-132.07 \\
-128.47 \\
-124.87 \\
-121.27 \\
-117.67 \\
-114.07 \\
-110.47\end{array}$ & $\begin{array}{r}228.019 \\
462.208 \\
871.917 \\
1342.952 \\
1818.188 \\
2295.371 \\
2771.511 \\
3246.661 \\
3720.976 \\
4192.188 \\
4662.578\end{array}$ & $\begin{array}{l}105.382 \\
105.306 \\
105.093 \\
105.055 \\
105.024 \\
104.997 \\
104.971 \\
104.946 \\
104.923 \\
104.900 \\
104.877\end{array}$ \\
\hline $\begin{array}{r}-124.87 \\
-121.27 \\
-117.67 \\
-114.07 \\
-110.47 \\
-106.87 \\
-103.27 \\
-99.67 \\
-96.07 \\
-92.47 \\
-88.87 \\
-85.27\end{array}$ & $\begin{array}{r}375.455 \\
570.151 \\
984.196 \\
1412.300 \\
1842.407 \\
2273.133 \\
2703.906 \\
3133.688 \\
3562.011 \\
3989.277 \\
4413.368 \\
4837.426\end{array}$ & $\begin{array}{l}103.134 \\
102.914 \\
102.865 \\
102.831 \\
102.803 \\
102.777 \\
102.753 \\
102.730 \\
102.708 \\
102.686 \\
102.664 \\
102.643\end{array}$ \\
\hline $\begin{array}{l}-96.07 \\
-92.47 \\
-88.87 \\
-85.27 \\
-81.67 \\
-78.07 \\
-74.47 \\
-70.87 \\
-67.27 \\
-63.67 \\
-60.07 \\
-56.47 \\
-52.87 \\
-49.27 \\
-45.67\end{array}$ & $\begin{array}{r}224.476 \\
459.578 \\
740.755 \\
1104.099 \\
1472.018 \\
1841.267 \\
2210.821 \\
2579.685 \\
2947.419 \\
3314.253 \\
3680.126 \\
4045.609 \\
4409.976 \\
4772.590 \\
5133.771\end{array}$ & $\begin{array}{l}99.752 \\
99.677 \\
99.473 \\
99.437 \\
99.409 \\
99.383 \\
99.360 \\
99.338 \\
99.316 \\
99.296 \\
99.275 \\
99.255 \\
99.236 \\
99.216 \\
99.197\end{array}$ \\
\hline $\begin{array}{l}-63.67 \\
-56.47 \\
-49.27 \\
-42.07 \\
-34.87 \\
-27.67 \\
-20.47 \\
-13.27\end{array}$ & $\begin{array}{r}519.839 \\
1144.037 \\
1784.450 \\
2423.695 \\
3061.471 \\
3696.638 \\
4328.811 \\
4956.372\end{array}$ & $\begin{array}{l}96.326 \\
96.249 \\
96.200 \\
96.157 \\
96.117 \\
96.079 \\
96.042 \\
96.006\end{array}$ \\
\hline
\end{tabular}


Table 21(b) (continued). Isochoric PVT Measurements for HCFC-125 in PI units.

$\begin{array}{rcc}\text { Temperature } & \text { Pressure } & \begin{array}{c}\text { Density } \\ \text { Fahrenheit }\end{array} \\ \text { psia } & & \text { 1b -ms } / \text { ft }^{3} \\ -20.47 & 456.958 & 90.945 \\ -13.27 & 882.003 & 90.718 \\ -6.07 & 1381.229 & 90.670 \\ 1.13 & 1882.364 & 90.630 \\ 8.33 & 2383.147 & 90.593 \\ 15.53 & 2882.156 & 90.558 \\ 22.73 & 3379.432 & 90.524 \\ 29.93 & 3875.660 & 90.491 \\ 37.13 & 4369.393 & 90.458 \\ 44.33 & 4860.407 & 90.426 \\ & & \\ 37.13 & 386.426 & 82.528 \\ 44.33 & 665.345 & 82.290 \\ 51.53 & 1008.370 & 82.245 \\ 58.73 & 1353.286 & 82.208 \\ 65.93 & 1699.029 & 82.174 \\ 73.13 & 2045.017 & 82.143 \\ 80.33 & 2390.777 & 82.112 \\ 87.53 & 2736.336 & 82.083 \\ 94.73 & 3080.024 & 82.053 \\ 101.93 & 3424.000 & 82.025 \\ 109.13 & 3767.272 & 81.996 \\ 116.33 & 4110.393 & 81.968 \\ 123.53 & 4451.935 & 81.940 \\ 130.73 & 4791.144 & 81.912 \\ 137.93 & 5130.298 & 81.886 \\ 98.33 & 358.642 & 70.328 \\ 105.53 & 534.313 & 70.105 \\ 112.73 & 732.534 & 70.064 \\ 119.93 & 932.837 & 70.032 \\ 127.13 & 1134.653 & 70.003 \\ 134.33 & 1337.264 & 69.977 \\ 141.53 & 1540.596 & 69.951 \\ 148.73 & 1744.122 & 69.928 \\ 155.93 & 1948.707 & 69.905 \\ 170.33 & 2358.540 & 69.856 \\ 184.73 & 2768.952 & 69.808 \\ 199.13 & 3179.403 & 69.761 \\ 213.53 & 3589.283 & 69.714 \\ 227.93 & 3998.361 & 69.667 \\ 242.33 & 4406.032 & 69.619 \\ 256.73 & 4812.440 & 69.572\end{array}$


Table 22. Ebulliometric vapor pressure measurements for HFC-125 (R-125).

Vapor Pressure of Pentafluoroethane (RI25)

\begin{tabular}{ccccc}
$\begin{array}{c}\text { Point } \\
\text { Number }\end{array}$ & $\begin{array}{c}\mathrm{T} \\
(\mathrm{K})\end{array}$ & $\begin{array}{c}\mathrm{P} \\
(\mathrm{kPa})\end{array}$ & $\begin{array}{c}\mathrm{T} \\
(\mathrm{F})\end{array}$ & $\begin{array}{c}\text { P } \\
\text { (psia) }\end{array}$ \\
\hdashline 1 & 218.762 & 73.7126 & -65.898 & 10.691 \\
2 & 219.175 & 75.3215 & -65.154 & 10.925 \\
3 & 219.444 & 76.3836 & -64.671 & 11.079 \\
4 & 219.978 & 78.5190 & -63.710 & 11.388 \\
5 & 220.174 & 79.3263 & -63.357 & 11.505 \\
6 & 220.381 & 80.1 .824 & -62.985 & 11.630 \\
7 & 220.553 & 80.9071 & -62.674 & 11.735 \\
8 & 220.916 & 82.4188 & -62.022 & 11.954 \\
9 & 221.185 & 83.5673 & -61.537 & 12.120 \\
10 & 221.575 & 85.2409 & -60.836 & 12.363 \\
11 & 222.257 & 88.2381 & -59.608 & 12.798 \\
12 & 222.855 & 90.9315 & -58.531 & 13.189 \\
13 & 222.736 & 90.3914 & -58.745 & 13.110 \\
14 & 223.343 & 93.1839 & -57.652 & 13.515 \\
15 & 224.053 & 96.5393 & -56.375 & 14.002 \\
16 & 224.984 & 101.0860 & -54.698 & 14.661 \\
17 & 226.262 & 107.5412 & -52.398 & 15.598 \\
18 & 226.442 & 108.4831 & -52.074 & 15.734 \\
19 & 226.371 & 108.1079 & -52.203 & 15.680 \\
20 & 227.055 & 111.7351 & -50.971 & 16.206 \\
21 & 227.495 & 114.1230 & -50.178 & 16.552 \\
22 & 228.491 & 119.6434 & -48.387 & 17.353 \\
23 & 229.270 & 124.1224 & -46.983 & 18.002 \\
24 & 230.070 & 128.8654 & -45.543 & 18.690 \\
25 & 231.835 & 139.8029 & -42.367 & 20.277 \\
26 & 233.989 & 154.1359 & -38.491 & 22.356 \\
27 & 233.825 & 153.0023 & -38.786 & 22.191 \\
28 & 235.033 & 161.4451 & -36.611 & 23.416 \\
29 & 236.643 & 173.3154 & -33.713 & 25.137 \\
30 & 238.261 & 185.8698 & -30.800 & 26.958 \\
31 & 237.774 & 182.0049 & -31.678 & 26.398 \\
32 & 239.292 & 194.2497 & -28.945 & 28.174 \\
33 & 240.801 & 207.0155 & -26.228 & 30.025 \\
34 & 242.532 & 222.4285 & -23.113 & 32.261 \\
35 & 244.317 & 239.2461 & -19.900 & 34.700 \\
36 & 245.180 & 247.7090 & -18.347 & 35.927 \\
37 & 245.662 & 252.5508 & -17.479 & 36.630 \\
38 & 246.052 & 256.5198 & -16.777 & 37.205 \\
39 & 246.589 & 262.0412 & -15.809 & 38.006
\end{tabular}


Table 23a. Fit of the HFC-125 (R-125) vapor pressure measurements to an Antoine equation (SI units). The percent deviation is $100 \times\left(P-P_{\text {calc }}\right) / P_{\text {calc }}$.

\begin{tabular}{|c|c|c|c|c|}
\hline \multicolumn{3}{|c|}{ Pcalc $=\exp (A+(B /(T+C)))$} & \multicolumn{2}{|c|}{$\begin{array}{l}A=14.03892 \\
B=-1809.80 \\
C=-32.92627\end{array}$} \\
\hline $\begin{array}{l}\text { Point } \\
\text { Number }\end{array}$ & $\underset{(K)}{T}$ & $\begin{array}{c}\mathrm{P} \\
(\mathrm{kPa})\end{array}$ & $\begin{array}{r}\text { Pcalc } \\
(\mathrm{kPa})\end{array}$ & $\begin{array}{c}\text { Deviat } \\
\%\end{array}$ \\
\hline 1 & 218.762 & 73.713 & 73.715 & -0.003 \\
\hline 2 & 219.175 & 75.322 & 75.325 & -0.005 \\
\hline 3 & 219.444 & 76.384 & 76.386 & -0.003 \\
\hline 4 & 219.978 & 78.519 & 78.531 & -0.016 \\
\hline 5 & 220.174 & 79.326 & 79.331 & -0.006 \\
\hline 6 & 220.381 & 80.182 & 80.181 & 0.002 \\
\hline 7 & 220.553 & 80.907 & 80.898 & 0.012 \\
\hline 8 & 220.916 & 82.419 & 82.415 & 0.004 \\
\hline 9 & 221.185 & 83.567 & 83.559 & 0.010 \\
\hline 10 & 221.575 & 85.241 & 85.234 & 0.008 \\
\hline 11 & 222.257 & 88.238 & 88.232 & 0.007 \\
\hline 12 & 222.855 & 90.931 & 90.929 & 0.003 \\
\hline 13 & 222.736 & 90.391 & 90.388 & 0.004 \\
\hline 14 & 223.343 & 93.184 & 93.178 & 0.006 \\
\hline 15 & 224.053 & 96.539 & 96.525 & 0.015 \\
\hline 16 & 224.984 & 101.086 & 101.061 & 0.025 \\
\hline 17 & 226.262 & 107.541 & 107.556 & -0.014 \\
\hline 18 & 226.442 & 108.483 & 108.497 & -0.013 \\
\hline 19 & 226.371 & 108.108 & 108.122 & -0.013 \\
\hline 20 & 227.055 & 111.735 & 111.746 & -0.010 \\
\hline 21 & 227.495 & 114.123 & 114.130 & -0.006 \\
\hline 22 & 228.491 & 119.643 & 119.662 & -0.016 \\
\hline 23 & 229.270 & 124.122 & 124.141 & -0.015 \\
\hline 24 & 230.070 & 128.865 & 128.873 & -0.006 \\
\hline 25 & 231.835 & 139.803 & 139.808 & -0.004 \\
\hline 26 & 233.989 & 154.136 & 154.119 & 0.011 \\
\hline 27 & 233.825 & 153.002 & 152.991 & 0.007 \\
\hline 28 & 235.033 & 161.445 & 161.453 & -0.005 \\
\hline 29 & 236.643 & 173.315 & 173.293 & 0.013 \\
\hline 30 & 238.261 & 185.870 & 185.865 & 0.003 \\
\hline 31 & 237.774 & 182.005 & 182.006 & -0.001 \\
\hline 32 & 239.292 & 194.250 & 194.229 & 0.011 \\
\hline 33 & 240.801 & 207.015 & 206.997 & 0.009 \\
\hline 34 & 242.532 & 222.429 & 222.424 & 0.002 \\
\hline 35 & 244.317 & 239.246 & 239.248 & -0.001 \\
\hline 36 & 245.180 & 247.709 & 247.724 & -0.006 \\
\hline 37 & 245.662 & 252.551 & 252.555 & -0.002 \\
\hline 38 & 246.052 & 256.520 & 256.520 & -0.000 \\
\hline 39 & 246.589 & 262.041 & 262.059 & -0.007 \\
\hline
\end{tabular}


Table 23b. Fit of the HFC-125 (R-125) vapor pressure measurements to an Antoine equation (PI units). The percent deviation is $100 \times\left(P-P_{c-1 c}\right) / P_{c a l c}$.

Antoine equation $-\operatorname{Rl25}($. $g$. units $)$
Pcalc $=\exp (A+(B /(T+C)))$
$A=12.10816$
$B=-3257.64$
$B=400.40271$

\begin{tabular}{ccccc}
$\begin{array}{c}\text { Point } \\
\text { Number }\end{array}$ & T & P & $\begin{array}{c}\text { Pcalc } \\
\text { (psia) }\end{array}$ & \multicolumn{1}{c}{$\begin{array}{r}\text { Deviat } \\
\text { (psia) }\end{array}$} \\
\hline 1 & -65.898 & 10.691 & 10.691 & -0.003 \\
2 & -65.154 & 10.925 & 10.925 & -0.005 \\
3 & -64.671 & 11.079 & 11.079 & -0.003 \\
4 & -63.710 & 11.388 & 11.390 & -0.015 \\
5 & -63.357 & 11.505 & 11.506 & -0.006 \\
6 & -62.985 & 11.630 & 11.629 & 0.002 \\
7 & -62.674 & 11.735 & 11.733 & 0.012 \\
8 & -62.022 & 11.954 & 11.953 & 0.005 \\
9 & -61.537 & 12.120 & 12.119 & 0.010 \\
10 & -60.836 & 12.363 & 12.362 & 0.008 \\
11 & -59.608 & 12.798 & 12.797 & 0.007 \\
12 & -58.531 & 13.189 & 13.188 & 0.003 \\
13 & -58.745 & 13.110 & 13.110 & 0.004 \\
14 & -57.652 & 13.515 & 13.514 & 0.007 \\
15 & -56.375 & 14.002 & 14.000 & 0.015 \\
16 & -54.698 & 14.661 & 14.658 & 0.025 \\
17 & -52.398 & 15.598 & 15.600 & -0.014 \\
18 & -52.074 & 15.734 & 15.736 & -0.012 \\
19 & -52.203 & 15.680 & 15.682 & -0.013 \\
20 & -50.971 & 16.266 & 16.207 & -0.010 \\
21 & -50.178 & 16.552 & 16.553 & -0.005 \\
22 & -48.387 & 17.353 & 17.356 & -0.015 \\
23 & -46.983 & 18.002 & 18.005 & -0.015 \\
24 & -45.543 & 18.690 & 18.691 & -0.006 \\
25 & -42.367 & 20.277 & 20.277 & -0.004 \\
26 & -38.491 & 22.356 & 22.353 & 0.011 \\
27 & -38.786 & 22.191 & 22.190 & 0.008 \\
28 & -36.611 & 23.416 & 23.417 & -0.005 \\
29 & -33.713 & 25.137 & 25.134 & 0.013 \\
30 & -30.800 & 26.958 & 26.957 & 0.003 \\
31 & -31.678 & 26.398 & 26.398 & -0.000 \\
32 & -28.945 & 28.174 & 28.171 & 0.011 \\
33 & -26.228 & 30.025 & 30.022 & 0.009 \\
34 & -23.113 & 32.261 & 32.260 & 0.002 \\
35 & -19.900 & 34.700 & 34.700 & -0.000 \\
36 & -18.347 & 35.927 & 35.929 & -0.006 \\
37 & -17.479 & 36.630 & 36.630 & -0.001 \\
38 & -16.777 & 37.205 & 37.205 & 0.000 \\
39 & -15.809 & 38.006 & 38.008 & -0.007
\end{tabular}


Table 24(a). Isochoric PVT measurements for HCFC-123 in SI units.

\begin{tabular}{|c|c|}
\hline $\begin{array}{c}\text { Pressure } \\
\text { MPa }\end{array}$ & $\begin{array}{l}\text { Density } \\
\mathrm{mol} / \mathrm{dm}^{3}\end{array}$ \\
\hline $\begin{array}{r}3.932390 \\
7.843969 \\
11.739300 \\
15.603580 \\
19.436470 \\
23.250830 \\
27.043330 \\
30.799040 \\
34.545520\end{array}$ & $\begin{array}{l}11.45442 \\
11.45196 \\
11.44954 \\
11.44714 \\
11.44478 \\
11.44245 \\
11.44013 \\
11.43783 \\
11.43554\end{array}$ \\
\hline $\begin{array}{r}1.769352 \\
5.297392 \\
8.795440 \\
12.283070 \\
15.750070 \\
19.196620 \\
22.624520 \\
26.034240 \\
29.415060 \\
32.774780\end{array}$ & $\begin{array}{l}11.23640 \\
11.23405 \\
11.23174 \\
11.22947 \\
11.22721 \\
11.22498 \\
11.22276 \\
11.22056 \\
11.21838 \\
11.21621\end{array}$ \\
\hline $\begin{array}{r}2.124055 \\
5.273329 \\
8.411843 \\
11.535470 \\
14.637850 \\
17.722830 \\
20.796100 \\
23.850750 \\
26.891850 \\
29.917650 \\
32.929010\end{array}$ & $\begin{array}{l}11.00625 \\
11.00404 \\
11.00186 \\
10.99970 \\
10.99757 \\
10.99544 \\
10.99333 \\
10.99124 \\
10.98916 \\
10.98708 \\
10.98501\end{array}$ \\
\hline $\begin{array}{r}.434245 \\
1.810371 \\
4.549389 \\
7.273252 \\
9.938414 \\
12.691280 \\
15.380230 \\
18.056130 \\
20.727310 \\
23.377690 \\
26.022690 \\
28.650340 \\
31.271920 \\
33.876820\end{array}$ & $\begin{array}{l}10.71137 \\
10.71033 \\
10.70826 \\
10.70621 \\
10.70419 \\
10.70218 \\
10.70018 \\
10.69819 \\
10.69620 \\
10.69423 \\
10.69226 \\
10.69030 \\
10.68834 \\
10.68639\end{array}$ \\
\hline
\end{tabular}


Table 24(a) (continued). Isochoric PVT measurements for HCFC-123 in SI units.

$\begin{array}{lcc}\text { Temperature } & \text { Pressure } & \text { Density } \\ \text { Ke1vin } & \text { MPa } & \\ & & \\ 249.001 & .840899 & 10.35636 \\ 250.002 & 2.001339 & 10.35539 \\ 252.000 & 4.321377 & 10.35347 \\ 254.000 & 6.631163 & 10.35157 \\ 255.999 & 8.930861 & 10.34968 \\ 258.000 & 11.225400 & 10.34779 \\ 259.999 & 13.508870 & 10.34592 \\ 262.001 & 15.785470 & 10.34405 \\ 263.999 & 18.049410 & 10.34218 \\ 266.000 & 20.311120 & 10.34033 \\ 268.001 & 22.560400 & 10.33847 \\ 270.000 & 24.798100 & 10.33662 \\ 272.000 & 27.035190 & 10.33478 \\ 274.000 & 29.259860 & 10.33293 \\ 276.001 & 31.475760 & 10.33109 \\ 278.000 & 33.681210 & 10.32925 \\ 269.999 & 2.688618 & 10.05904 \\ 272.000 & 4.698253 & 10.05723 \\ 274.001 & 6.704591 & 10.05543 \\ 275.998 & 8.712440 & 10.05363 \\ 278.002 & 10.701840 & 10.05184 \\ 280.002 & 12.696030 & 10.05005 \\ 282.001 & 14.687050 & 10.04828 \\ 284.001 & 16.671610 & 10.04650 \\ 286.002 & 18.649080 & 10.04473 \\ 288.001 & 20.612280 & 10.04296 \\ 290.002 & 22.580660 & 10.04119 \\ 291.998 & 24.538400 & 10.03942 \\ 293.999 & 26.489790 & 10.03765 \\ 296.000 & 28.434620 & 10.03589 \\ 298.001 & 30.368990 & 10.03412 \\ 300.000 & 32.300790 & 10.03236 \\ 302.001 & 34.214710 & 10.03060 \\ 298.001 & 3.166692 & \\ 300.001 & 4.822544 & 9.62720 \\ 302.000 & 6.475250 & 9.62552 \\ 303.999 & 8.125226 & 9.62384 \\ 306.001 & 9.771155 & 9.62217 \\ 307.998 & 11.406540 & 9.62049 \\ 310.001 & 13.050340 & 9.61885 \\ 312.001 & 14.683990 & 9.61715 \\ 313.999 & 16.310520 & 9.61549 \\ 316.001 & 17.932590 & 9.61382 \\ 320.000 & 21.172570 & 9.61216 \\ 324.000 & 24.392040 & 9.60883 \\ 328.001 & 27.589630 & 9.60549 \\ 332.001 & 30.772140 & 9.60217 \\ & & 9.59883\end{array}$


Table 24(a) (continued). Isochoric PVT measurements for HCFC-123 in SI units.

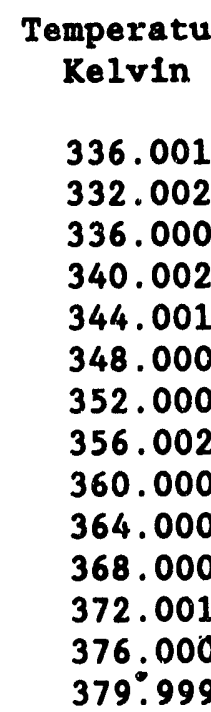

\section{Pressure MPa}

33.932900

2.588553

5.132132

7.654527

10.190930

12.699880

15.210770

17.703940

20.191730

22.681310

25.146830

27.601790

30.049470 .

32.487460
Density $\mathrm{mol} / \mathrm{dm}^{3}$

9.59549

9.04028

9.03719

9.03426

9.03104

9.02799

9.02503

9.02200

9.01891

9.01580

9.01269

9.00957

9.00644

9.00331 
Table 24(b). Isochoric PVT measurements for HCFC-123 in PI units.

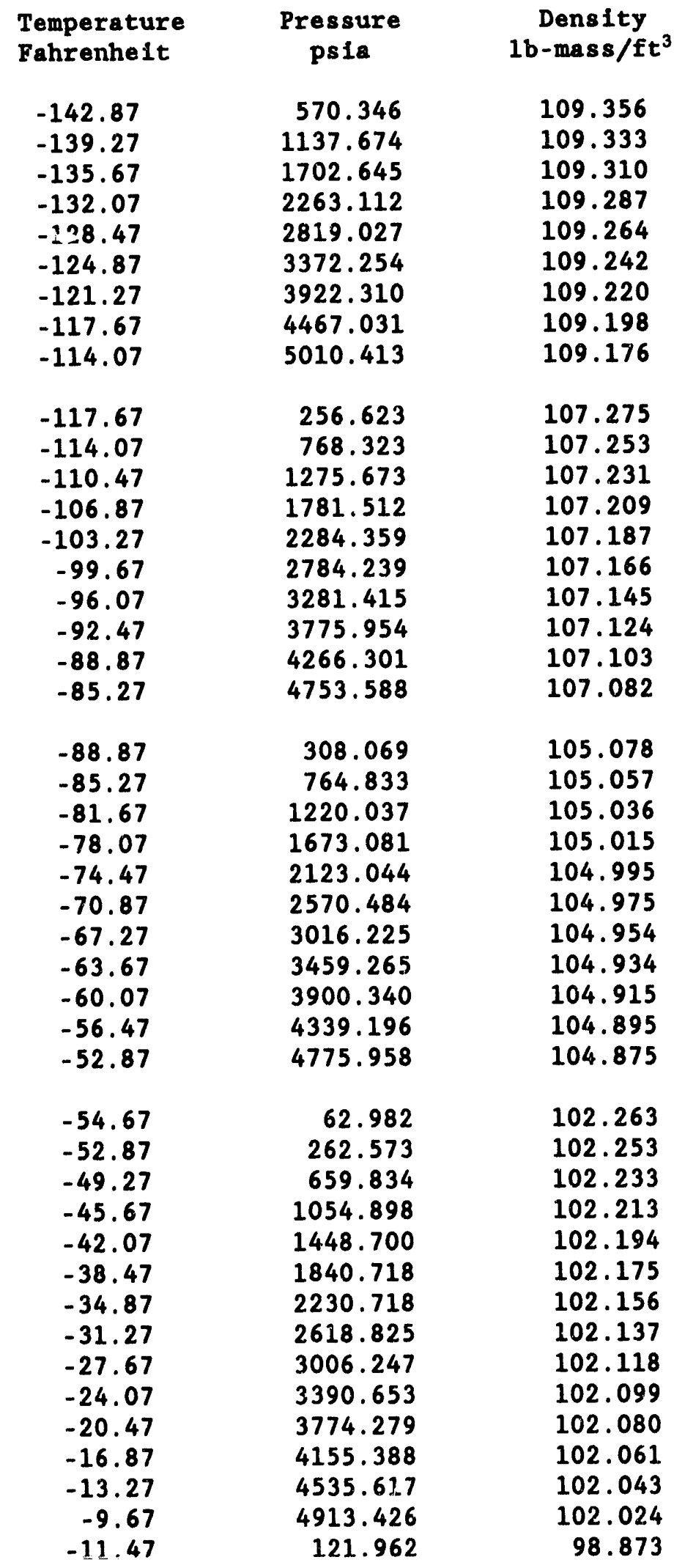


Table 24(b) (continued). Isochoric PVT measurements for HCFC-123 in PI units.

\begin{tabular}{|c|c|c|}
\hline $\begin{array}{l}\text { Temperature } \\
\text { Fahrenheit }\end{array}$ & $\begin{array}{l}\text { Pressure } \\
\text { psia }\end{array}$ & $\begin{array}{c}\text { Density } \\
1 \mathrm{~b} \text {-mass/ft }\end{array}$ \\
\hline $\begin{array}{r}-9.67 \\
-6.07 \\
-2.47 \\
1.13 \\
4.73 \\
8.33 \\
11.93 \\
15.53 \\
19.13 \\
22.73 \\
26.33 \\
29.93 \\
33.53 \\
37.13 \\
40.73\end{array}$ & $\begin{array}{r}290.270 \\
626.764 \\
961.771 \\
1295.314 \\
1628.110 \\
1959.299 \\
2289.493 \\
2617.850 \\
2945.884 \\
3272.115 \\
3596.667 \\
3921.130 \\
4243.791 \\
4565.181 \\
4885.055\end{array}$ & $\begin{array}{l}98.864 \\
98.846 \\
98.827 \\
98.809 \\
98.791 \\
98.774 \\
98.756 \\
98.738 \\
98.720 \\
98.702 \\
98.685 \\
98.667 \\
98.650 \\
98.632 \\
98.614\end{array}$ \\
\hline $\begin{array}{l}26.33 \\
29.93 \\
33.53 \\
37.13 \\
40.73 \\
44.33 \\
47.93 \\
51.53 \\
55.13 \\
58.73 \\
62.33 \\
65.93 \\
69.53 \\
73.13 \\
76.73 \\
80.33 \\
83.93\end{array}$ & $\begin{array}{r}389.952 \\
681.425 \\
972.420 \\
1263.635 \\
1552.173 \\
1841.407 \\
2130.180 \\
2418.017 \\
2704.825 \\
2989.564 \\
3275.054 \\
3559.000 \\
3842.026 \\
4124.100 \\
4404.657 \\
4684.842 \\
4962.433\end{array}$ & $\begin{array}{l}96.035 \\
96.017 \\
96.000 \\
95.983 \\
95.966 \\
95.949 \\
95.932 \\
95.915 \\
95.898 \\
95.881 \\
95.864 \\
95.847 \\
95.830 \\
95.814 \\
95.797 \\
95.780 \\
95.763\end{array}$ \\
\hline $\begin{array}{r}76.73 \\
80.33 \\
83.93 \\
87.53 \\
91.13 \\
94.73 \\
98.33 \\
101.93 \\
105.53 \\
109.13 \\
116.33 \\
123.53 \\
130.73 \\
137.93 \\
145.13\end{array}$ & $\begin{array}{r}459.291 \\
699.452 \\
939.157 \\
1178.466 \\
1417.189 \\
1654.382 \\
1892.795 \\
2129.736 \\
2365.645 \\
2600.907 \\
3070.827 \\
3537.773 \\
4001.545 \\
4463.129 \\
4921.560\end{array}$ & $\begin{array}{l}91.912 \\
91.896 \\
91.880 \\
91.864 \\
91.848 \\
91.832 \\
91.816 \\
91.800 \\
91.784 \\
91.768 \\
91.736 \\
91.705 \\
91.673 \\
91.641 \\
91.609\end{array}$ \\
\hline
\end{tabular}


Table 24(b) (continued). Isochoric PVT measurements for HCFC-123 in PI units.

$\begin{array}{ccr}\begin{array}{c}\text { Temperstuxe } \\ \text { Fahrenhelt }\end{array} & \begin{array}{c}\text { Pressure } \\ \text { psia }\end{array} & \begin{array}{c}\text { Density } \\ \text { 1b-mass/ft }\end{array} \\ 137.93 & 375.439 & 86.308 \\ 145.13 & 744.354 & 86.279 \\ 152.33 & 1110.197 & 86.251 \\ 159.53 & 1478.072 & 86.220 \\ 166.73 & 1841.965 & 86.191 \\ 173.93 & 2206.140 & 86.163 \\ 181.13 & 2567.744 & 86.134 \\ 188.33 & 2928.568 & 86.104 \\ 195.53 & 3289.652 & 86.075 \\ 202.73 & 3647.246 & 86.045 \\ 209.93 & 4003.308 & 86.015 \\ 217.13 & 4358.315 & 85.985 \\ 224.33 & 4711.916 & 85.956\end{array}$


Table 25(a). Experimental 1iquid heat capacity data for HCFC-123.

\begin{tabular}{|c|c|c|c|c|c|c|c|c|c|}
\hline $\mathbf{T}$ & $\delta \mathrm{T}$ & $\rho$ & $\delta \rho$ & $\mathbf{P}$ & $\delta \mathbf{P}$ & $\bar{C}_{v, \exp }$ & $\delta \mathbf{C}_{\mathbf{v}}$ & $\delta \mathrm{C}_{\mathrm{v}}, 8$ & $\overline{\text { Run }}$ \\
\hline \multicolumn{2}{|c|}{$\mathbf{K}$} & \multicolumn{2}{|c|}{$\mathrm{mol} \mathrm{dm}^{-3}$} & \multicolumn{2}{|c|}{$\mathbf{M P a}$} & \multicolumn{2}{|c|}{$\mathrm{J} \operatorname{mol}^{-1} \mathrm{~K}^{-1}$} & & \\
\hline $\begin{array}{l}257.8615 \\
259.8746 \\
261.9246 \\
263.9040 \\
265.9697 \\
267.9195 \\
269.9803 \\
271.9136 \\
273.9567 \\
275.8598 \\
277.9191 \\
279.7909 \\
283.6947 \\
287.5835\end{array}$ & $\begin{array}{l}0.0034 \\
0.0032 \\
0.0035 \\
0.0033 \\
0.0036 \\
0.0031 \\
0.0034 \\
0.0032 \\
0.0033 \\
0.0032 \\
0.0032 \\
0.0032 \\
0.0032 \\
0.0033\end{array}$ & $\begin{array}{l}10.241 \\
10.237 \\
10.232 \\
10.228 \\
10.224 \\
10.220 \\
10.216 \\
10.212 \\
10.208 \\
10.204 \\
10.200 \\
10.196 \\
10.188 \\
10.180\end{array}$ & $\begin{array}{l}0.009 \\
0.010 \\
0.011 \\
0.011 \\
0.012 \\
0.013 \\
0.014 \\
0.014 \\
0.015 \\
0.016 \\
0.017 \\
0.017 \\
0.019 \\
0.020\end{array}$ & $\begin{array}{r}3.163 \\
5.244 \\
7.357 \\
9.390 \\
11.502 \\
13.484 \\
15.565 \\
17.504 \\
19.539 \\
21.420 \\
23.440 \\
25.263 \\
29.024 \\
32.720\end{array}$ & $\begin{array}{l}0.033 \\
0.033 \\
0.033 \\
0.033 \\
0.033 \\
0.033 \\
0.033 \\
0.033 \\
0.033 \\
0.033 \\
0.033 \\
0.033 \\
0.033 \\
0.033\end{array}$ & $\begin{array}{l}103.93 \\
104.12 \\
105.04 \\
105.11 \\
106.26 \\
106.39 \\
106.68 \\
106.51 \\
107.52 \\
107.07 \\
108.29 \\
108.26 \\
109.19 \\
110.20\end{array}$ & $\begin{array}{l}0.64 \\
0.39 \\
0.29 \\
0.25 \\
0.22 \\
0.21 \\
0.20 \\
0.19 \\
0.19 \\
0.18 \\
0.18 \\
0.18 \\
0.17 \\
0.17\end{array}$ & $\begin{array}{l}0.62 \\
0.38 \\
0.28 \\
0.24 \\
0.21 \\
0.19 \\
0.19 \\
0.18 \\
0.17 \\
0.17 \\
0.16 \\
0.16 \\
0.16 \\
0.16\end{array}$ & $\begin{array}{l}a \\
b \\
a \\
b \\
a \\
b \\
a \\
b \\
a \\
b \\
a \\
b \\
b \\
b\end{array}$ \\
\hline
\end{tabular}

Table 25(b). Experimental liquid heat capacity data for HCFC-123.

\begin{tabular}{|c|c|c|c|c|c|c|c|c|c|}
\hline $\mathbf{T}$ & $\delta \mathbf{T}$ & $\rho$ & $\delta \rho$ & $\mathbf{P}$ & $\delta P$ & $\mathrm{C}_{\mathrm{v}, \text { exp }}$ & $\delta \mathrm{C}_{\mathrm{v}}$ & $\delta C_{v}, 8$ & Run \\
\hline \multicolumn{2}{|c|}{${ }^{\circ} \mathrm{F}$} & \multicolumn{2}{|c|}{$1 b \mathrm{ft}^{-3}$} & \multicolumn{2}{|c|}{ psia } & \multicolumn{3}{|c|}{ Btu $1 b^{-1}{ }^{\circ} F^{-1}$} & \\
\hline $\begin{array}{r}4.481 \\
8.104 \\
11.794 \\
15.357 \\
19.075 \\
22.585 \\
26.295 \\
29.774 \\
33.452 \\
36.878 \\
40.584 \\
43.954 \\
50.980 \\
57.980\end{array}$ & $\begin{array}{l}0.006 \\
0.006 \\
0.006 \\
0.006 \\
0.006 \\
0.006 \\
0.006 \\
0.006 \\
0.006 \\
0.006 \\
0.006 \\
0.006 \\
0.006 \\
0.006\end{array}$ & $\begin{array}{l}97.77 \\
97.73 \\
97.69 \\
97.65 \\
97.61 \\
97.57 \\
97.53 \\
97.49 \\
97.46 \\
97.42 \\
97.38 \\
97.34 \\
97.27 \\
97.19\end{array}$ & $\begin{array}{l}0.09 \\
0.10 \\
0.11 \\
0.11 \\
0.11 \\
0.12 \\
0.13 \\
0.13 \\
0.14 \\
0.15 \\
0.16 \\
0.16 \\
0.18 \\
0.19\end{array}$ & $\begin{array}{r}458.8 \\
760.6 \\
1067.0 \\
1361.9 \\
1668.2 \\
1955.7 \\
2257.5 \\
2538.7 \\
2833.9 \\
3106.7 \\
3399.7 \\
3664.1 \\
4209.6 \\
4745.6\end{array}$ & $\begin{array}{l}4.8 \\
4.8 \\
4.8 \\
4.8 \\
4.8 \\
4.8 \\
4.8 \\
4.8 \\
4.8 \\
4.8 \\
4.8 \\
4.8 \\
4.8 \\
4.8\end{array}$ & $\begin{array}{l}0.16243 \\
0.16272 \\
0.16416 \\
0.16427 \\
0.16607 \\
0.16627 \\
0.16672 \\
0.16646 \\
0.16804 \\
0.16733 \\
0.16924 \\
0.16919 \\
0.17065 \\
0.17223\end{array}$ & $\begin{array}{l}0.00100 \\
0.00061 \\
0.00045 \\
0.00039 \\
0.00034 \\
0.00033 \\
0.00031 \\
0.00030 \\
0.00030 \\
0.00028 \\
0.00028 \\
0.00028 \\
0.00027 \\
0.00027\end{array}$ & $\begin{array}{l}0.62 \\
0.38 \\
0.28 \\
0.24 \\
0.21 \\
0.19 \\
0.19 \\
0.18 \\
0.17 \\
0.17 \\
0.16 \\
0.16 \\
0.16 \\
0.16\end{array}$ & $\begin{array}{l}a \\
b \\
a \\
b \\
a \\
b \\
a \\
b \\
a \\
b \\
a \\
b \\
b \\
b\end{array}$ \\
\hline
\end{tabular}


Table 26(a). Experimental liquid heat capacity data for HCFC-123.

\begin{tabular}{|c|c|c|c|c|c|c|c|c|c|}
\hline $\mathbf{T}$ & $\delta \mathbf{T}$ & $\rho$ & $\delta \rho$ & $\mathbf{P}$ & $\delta P$ & $C_{v, \exp }$ & $\delta C_{v}$ & $\delta C_{v}, 8$ & Run \\
\hline \multicolumn{2}{|c|}{$\overline{\mathbf{K}}$} & mo1 & $\mathrm{dm}^{-3}$ & \multicolumn{2}{|c|}{$\mathrm{MPa}$} & \multicolumn{2}{|c|}{$\mathrm{J} \mathrm{mol}^{-1} \mathrm{k}^{-1}$} & & \\
\hline $\begin{array}{l}284.2425 \\
286.1803 \\
288.2634 \\
290.1925 \\
292.2663 \\
294.1864 \\
296.2546 \\
298.1617 \\
300.2107 \\
302.1200 \\
304.1621 \\
306.0576 \\
308.0901 \\
309.9691 \\
311.9977 \\
313.8777\end{array}$ & $\begin{array}{l}0.0035 \\
0.0031 \\
0.0037 \\
0.0032 \\
0.0032 \\
0.0032 \\
0.0032 \\
0.0031 \\
0.0032 \\
0.0032 \\
0.0033 \\
0.0031 \\
0.0034 \\
0.0033 \\
0.0033 \\
0.0032\end{array}$ & $\begin{array}{l}9.846 \\
9.843 \\
9.839 \\
9.836 \\
9.832 \\
9.828 \\
9.825 \\
9.821 \\
9.818 \\
9.814 \\
9.810 \\
9.807 \\
9.803 \\
9.800 \\
9.797 \\
9.793\end{array}$ & $\begin{array}{l}0.009 \\
0.009 \\
0.010 \\
0.011 \\
0.011 \\
0.012 \\
0.013 \\
0.013 \\
0.014 \\
0.014 \\
0.015 \\
0.016 \\
0.016 \\
0.017 \\
0.017 \\
0.018\end{array}$ & $\begin{array}{r}3.642 \\
5.340 \\
7.157 \\
8.832 \\
10.624 \\
12.275 \\
14.044 \\
15.668 \\
17.404 \\
19.013 \\
20.725 \\
22.306 \\
23.992 \\
25.542 \\
27.206 \\
28.738\end{array}$ & $\begin{array}{l}0.012 \\
0.012 \\
0.012 \\
0.012 \\
0.012 \\
0.012 \\
0.012 \\
0.012 \\
0.012 \\
0.012 \\
0.012 \\
0.012 \\
0.012 \\
0.012 \\
0.012 \\
0.012\end{array}$ & $\begin{array}{l}108.44 \\
108.36 \\
109.32 \\
109.06 \\
110.08 \\
109.76 \\
110.48 \\
110.60 \\
111.31 \\
111.02 \\
111.47 \\
111.52 \\
111.57 \\
111.91 \\
112.82 \\
113.25\end{array}$ & $\begin{array}{l}0.54 \\
0.35 \\
0.28 \\
0.23 \\
0.21 \\
0.19 \\
0.18 \\
0.18 \\
0.17 \\
0.17 \\
0.17 \\
0.16 \\
0.17 \\
0.16 \\
0.16 \\
0.16\end{array}$ & $\begin{array}{l}0.50 \\
0.33 \\
0.25 \\
0.21 \\
0.19 \\
0.18 \\
0.17 \\
0.16 \\
0.16 \\
0.15 \\
0.15 \\
0.15 \\
0.15 \\
0.15 \\
0.15 \\
0.14\end{array}$ & $\begin{array}{l}a \\
b \\
a \\
b \\
a \\
b \\
a \\
b \\
a \\
b \\
a \\
b \\
a \\
b \\
a \\
b\end{array}$ \\
\hline
\end{tabular}

Table 26(b). Experimental liquid heat capacity data for HCFC-123.

\begin{tabular}{|c|c|c|c|c|c|c|c|c|c|}
\hline$T$ & $\delta T$ & $\rho$ & $\delta \rho$ & $\mathbf{P}$ & $\delta \mathbf{P}$ & $C_{v, \exp }$ & $\delta C_{v}$ & $\delta C_{v}, 8$ & Run \\
\hline \multicolumn{2}{|c|}{${ }^{\circ} \mathbf{F}$} & \multicolumn{2}{|c|}{$1 \mathrm{~b} \mathrm{ft}^{-3}$} & \multicolumn{2}{|c|}{ psia } & \multicolumn{4}{|c|}{ Btu $1 b^{-1}{ }^{\circ} F^{-1}$} \\
\hline $\begin{array}{r}51.966 \\
55.455 \\
59.204 \\
62.676 \\
66.409 \\
69.865 \\
73.588 \\
77.021 \\
80.709 \\
84.146 \\
87.822 \\
91.234 \\
94.892 \\
98.274 \\
101.926 \\
105.310\end{array}$ & $\begin{array}{l}0.006 \\
0.006 \\
0.007 \\
0.006 \\
0.006 \\
0.006 \\
0.006 \\
0.006 \\
0.006 \\
0.006 \\
0.006 \\
0.006 \\
0.006 \\
0.006 \\
0.006 \\
0.006\end{array}$ & $\begin{array}{l}94.00 \\
93.97 \\
93.93 \\
93.91 \\
93.87 \\
93.83 \\
93.80 \\
93.76 \\
93.73 \\
93.70 \\
93.66 \\
93.63 \\
93.59 \\
93.56 \\
93.53 \\
93.49\end{array}$ & $\begin{array}{l}0.09 \\
0.09 \\
0.10 \\
0.11 \\
0.11 \\
0.11 \\
0.12 \\
0.12 \\
0.13 \\
0.13 \\
0.14 \\
0.15 \\
0.15 \\
0.16 \\
0.16 \\
0.17\end{array}$ & $\begin{array}{r}528.2 \\
774.5 \\
1038.0 \\
1281.0 \\
1540.9 \\
1780.3 \\
2036.9 \\
2272.5 \\
2524.2 \\
2757.6 \\
3005.9 \\
3235.2 \\
3479.8 \\
3704.6 \\
3945.9 \\
4168.1\end{array}$ & $\begin{array}{l}1.7 \\
1.7 \\
1.7 \\
1.7 \\
1.7 \\
1.7 \\
1.7 \\
1.7 \\
1.7 \\
1.7 \\
1.7 \\
1.7 \\
1.7 \\
1.7 \\
1.7 \\
1.7\end{array}$ & $\begin{array}{l}0.16947 \\
0.16935 \\
0.17085 \\
0.17044 \\
0.17204 \\
0.17154 \\
0.17266 \\
0.17285 \\
0.17396 \\
0.17351 \\
0.17421 \\
0.17429 \\
0.17437 \\
0.17490 \\
0.17632 \\
0.17699\end{array}$ & $\begin{array}{l}0.00084 \\
0.00055 \\
0.00044 \\
0.00036 \\
0.00033 \\
0.00030 \\
0.00028 \\
0.00028 \\
0.00027 \\
0.00027 \\
0.00027 \\
0.00025 \\
0.00027 \\
0.00025 \\
0.00025 \\
0.00025\end{array}$ & $\begin{array}{l}0.50 \\
0.33 \\
0.25 \\
0.21 \\
0.19 \\
0.18 \\
0.17 \\
0.16 \\
0.16 \\
0.15 \\
0.15 \\
0.15 \\
0.15 \\
0.15 \\
0.15 \\
0.14\end{array}$ & $\begin{array}{l}a \\
b \\
a \\
b \\
a \\
b \\
a \\
b \\
a \\
b \\
a \\
b \\
a \\
b \\
a \\
b\end{array}$ \\
\hline
\end{tabular}


Table 27(a). Experimental liquid heat capacity data for HCFC-123.

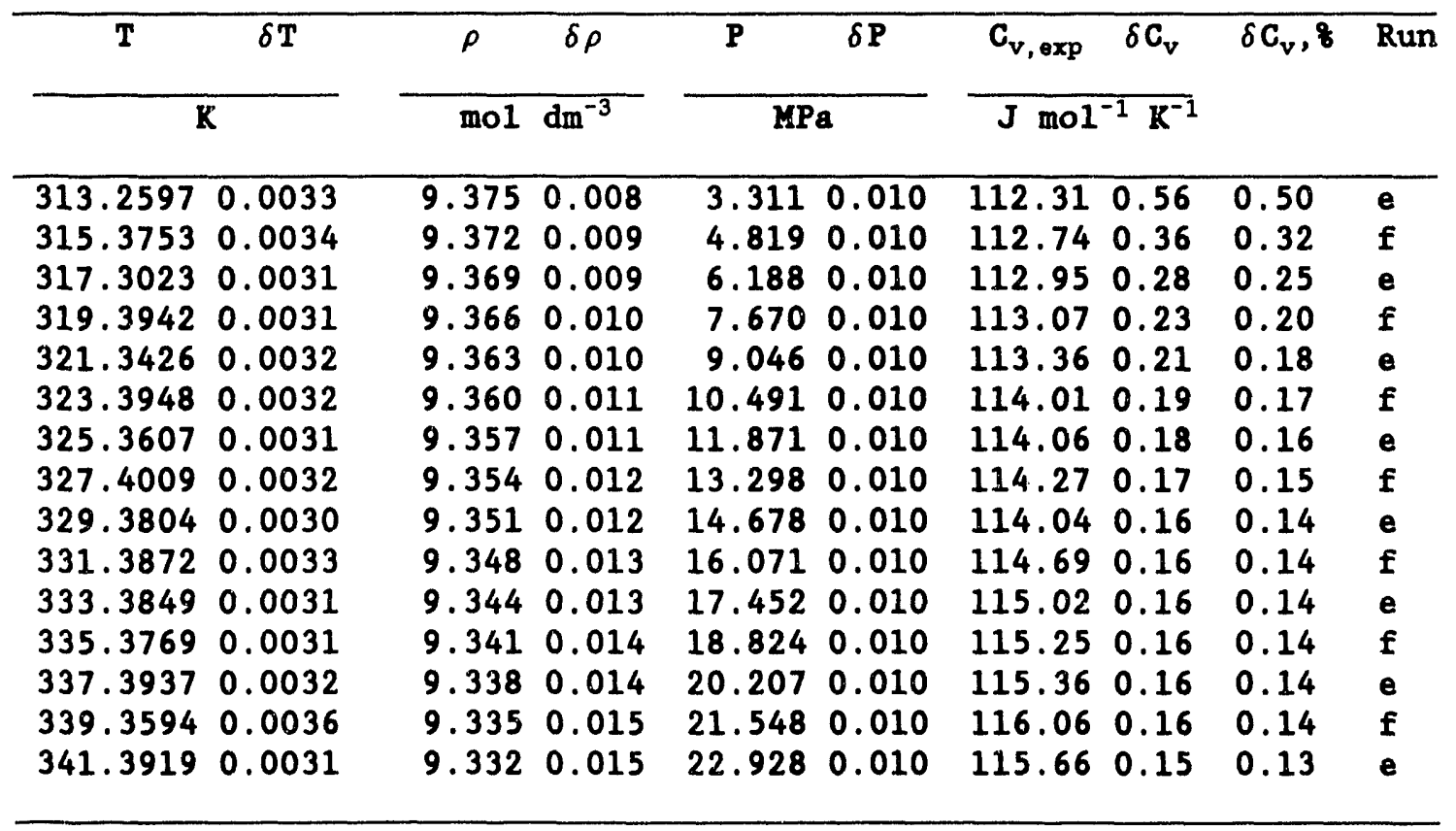

Table 27(b). Experimental liquid heat capacity data for HCFC-123.

\begin{tabular}{|c|c|c|c|c|c|c|c|c|c|}
\hline $\mathbf{T}$ & $\delta T$ & $\rho$ & $\delta \rho$ & $\mathbf{P}$ & $\delta \mathbf{P}$ & $C_{v, \exp }$ & $\delta \mathrm{C}_{\mathrm{v}}$ & $\delta \mathrm{C}_{\mathrm{v}}, \mathrm{z}$ & Run \\
\hline \multicolumn{2}{|c|}{${ }^{\circ} \mathrm{F}$} & \multicolumn{2}{|c|}{$\overline{1 b \mathrm{ft}^{-3}}$} & \multicolumn{2}{|c|}{ psia } & \multicolumn{4}{|c|}{ Btu $1 b^{-1}{ }^{\circ} F^{-1}$} \\
\hline 104.197 & 0.006 & 89.50 & 0.08 & 480.2 & 1.5 & 0.17552 & 0.00088 & 0.50 & e \\
\hline 108.006 & 0.006 & 89.48 & 0.09 & 698.9 & 1.5 & 0.17619 & 0.00056 & 0.32 & $\mathbf{f}$ \\
\hline 111.474 & 0.006 & 89.45 & 0.09 & 897.5 & 1.5 & 0.17652 & 0.00044 & 0.25 & e \\
\hline 115.240 & 0.006 & 89.42 & 0.10 & 1112.4 & 1.5 & 0.17671 & 0.00036 & 0.20 & $\mathbf{f}$ \\
\hline 118.747 & 0.006 & 89.39 & 0.10 & 1312.0 & 1.5 & 0.17716 & 0.00033 & 0.18 & e \\
\hline 122.441 & 0.006 & 89.36 & 0.11 & 1521.6 & 1.5 & 0.17818 & 0.00030 & 0.17 & $\mathbf{f}$ \\
\hline 125.979 & 0.006 & 89.33 & 0.11 & 1721.7 & 1.5 & 0.17826 & 0.00028 & 0.16 & e \\
\hline 129.652 & 0.006 & 89.30 & 0.11 & 1928.7 & 1.5 & 0.17859 & 0.00027 & 0.15 & $\mathbf{f}$ \\
\hline 133.215 & 0.005 & 89.27 & 0.11 & 2128.9 & 1.5 & 0.17823 & 0.00025 & 0.14 & e \\
\hline 136.827 & 0.006 & 89.2 .5 & 0.12 & 2330.9 & 1.5 & 0.17924 & 0.00025 & 0.14 & $\mathbf{f}$ \\
\hline 140.423 & 0.006 & 89.21 & 0.12 & 2531.2 & 1.5 & 0.17976 & 0.00025 & 0.14 & e \\
\hline 144.008 & 0.006 & 80.18 & 0.13 & 2730.2 & 1.5 & 0.18012 & 0.00025 & 0.14 & $\mathbf{f}$ \\
\hline 147.639 & 0.006 & 89.15 & 0.13 & 2930.8 & 1.5 & 0.18029 & 0.00025 & 0.14 & e \\
\hline 151.177 & 0.006 & 89.12 & 0.14 & 3125.3 & 1.5 & 0.18138 & 0.00025 & 0.14 & $\mathbf{f}$ \\
\hline 154.835 & 0.006 & 89.09 & 0.14 & 3325.4 & 1.5 & 0.18076 & 0.00023 & 0.13 & e \\
\hline
\end{tabular}


Table 28(a). Experimental liquid heat capacity data for HCFC-123.

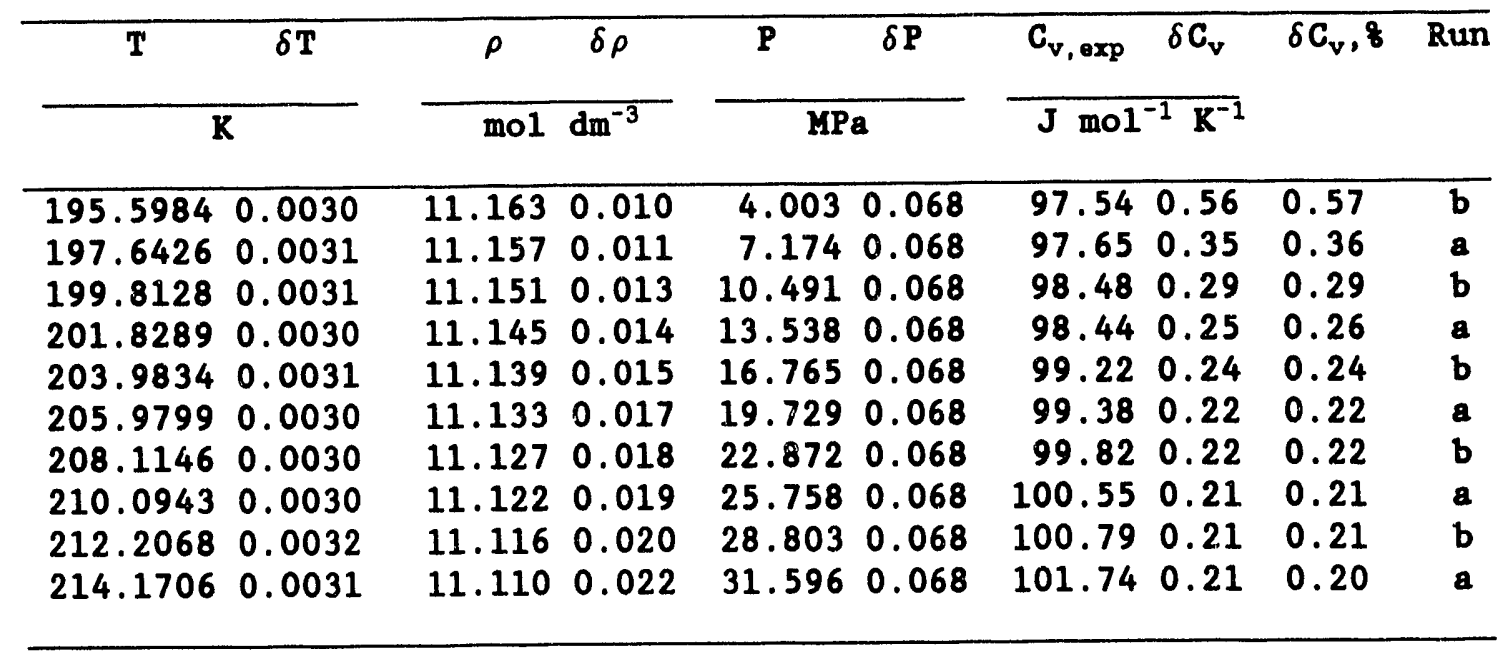

Table 28(b). Experimental liquid heat capacity data for HCFC-123.

\begin{tabular}{|c|c|c|c|c|c|c|c|c|c|}
\hline $\mathbf{T}$ & $\delta \mathbf{T}$ & $\rho$ & $\delta \rho$ & $\mathbf{P}$ & $\delta \mathrm{P}$ & $C_{\mathrm{v}, \exp }$ & $\delta \mathrm{C}_{\mathrm{v}}$ & $\delta C_{v}, 8$ & Run \\
\hline \multicolumn{2}{|c|}{${ }^{\circ} \mathrm{F}$} & \multicolumn{2}{|c|}{ Ib $\mathrm{ft}^{-3}$} & \multicolumn{2}{|c|}{ psia } & \multicolumn{4}{|c|}{ Btu $1 b^{-1}{ }^{\circ} F^{-1}$} \\
\hline $\begin{array}{r}-107.593 \\
-103.913 \\
-100.007 \\
-96.378 \\
-92.500 \\
-88.906 \\
-85.064 \\
-81.500 \\
-77.698 \\
-74.163\end{array}$ & $\begin{array}{l}0.005 \\
0.006 \\
0.006 \\
0.005 \\
0.006 \\
0.005 \\
0.005 \\
0.005 \\
0.006 \\
0.006\end{array}$ & $\begin{array}{l}106.57 \\
106.52 \\
106.46 \\
106.40 \\
106.35 \\
106.29 \\
106.23 \\
106.18 \\
106.13 \\
106.07\end{array}$ & $\begin{array}{l}0.10 \\
0.11 \\
0.12 \\
0.13 \\
0.14 \\
0.16 \\
0.17 \\
0.18 \\
0.19 \\
0.21\end{array}$ & $\begin{array}{r}580.6 \\
1040.5 \\
1521.6 \\
1963.5 \\
2431.6 \\
2861.5 \\
3317.3 \\
3735.9 \\
4177.5 \\
4582.6\end{array}$ & $\begin{array}{l}9.9 \\
9.9 \\
9.9 \\
9.9 \\
9.9 \\
9.9 \\
9.9 \\
9.9 \\
9.9 \\
9.9\end{array}$ & $\begin{array}{l}0.15244 \\
0.15261 \\
0.15391 \\
0.15385 \\
0.15507 \\
0.15532 \\
0.15600 \\
0.15714 \\
0.15752 \\
0.15900\end{array}$ & $\begin{array}{l}0.00088 \\
0.00055 \\
0.00045 \\
0.00039 \\
0.00038 \\
0.00034 \\
0.00034 \\
0.00033 \\
0.00033 \\
0.00033\end{array}$ & $\begin{array}{l}0.57 \\
0.36 \\
0.29 \\
0.26 \\
0.24 \\
0.22 \\
0.22 \\
0.21 \\
0.21 \\
0.20\end{array}$ & $\begin{array}{l}\mathrm{b} \\
\mathrm{a} \\
\mathrm{b} \\
\mathrm{a} \\
\mathrm{b} \\
\mathrm{a} \\
\mathrm{b} \\
\mathrm{a} \\
\mathrm{b} \\
\mathrm{a}\end{array}$ \\
\hline
\end{tabular}


Table 29(a). Experimental liquid heat capacity data for HCFC-123.

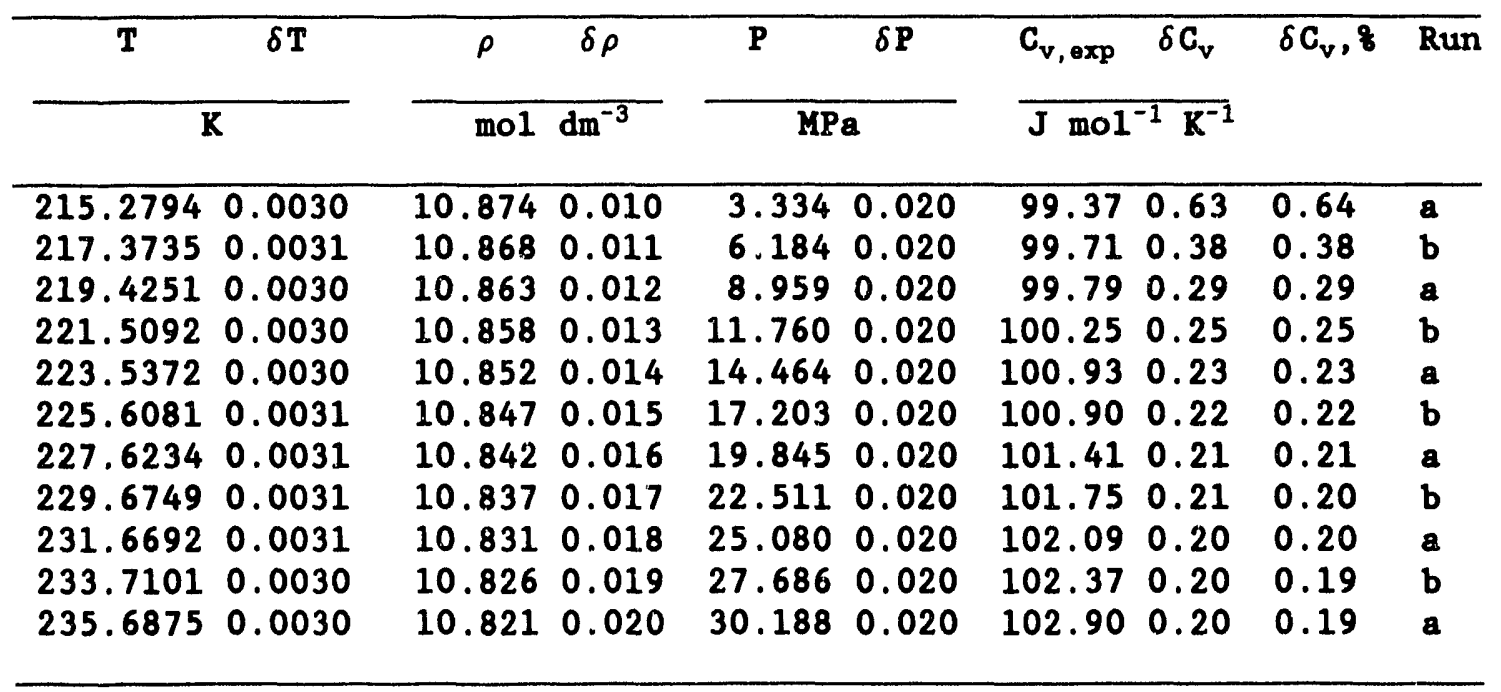

Table 29(b). Experimental liquid heat capacity data for HCFC-123.

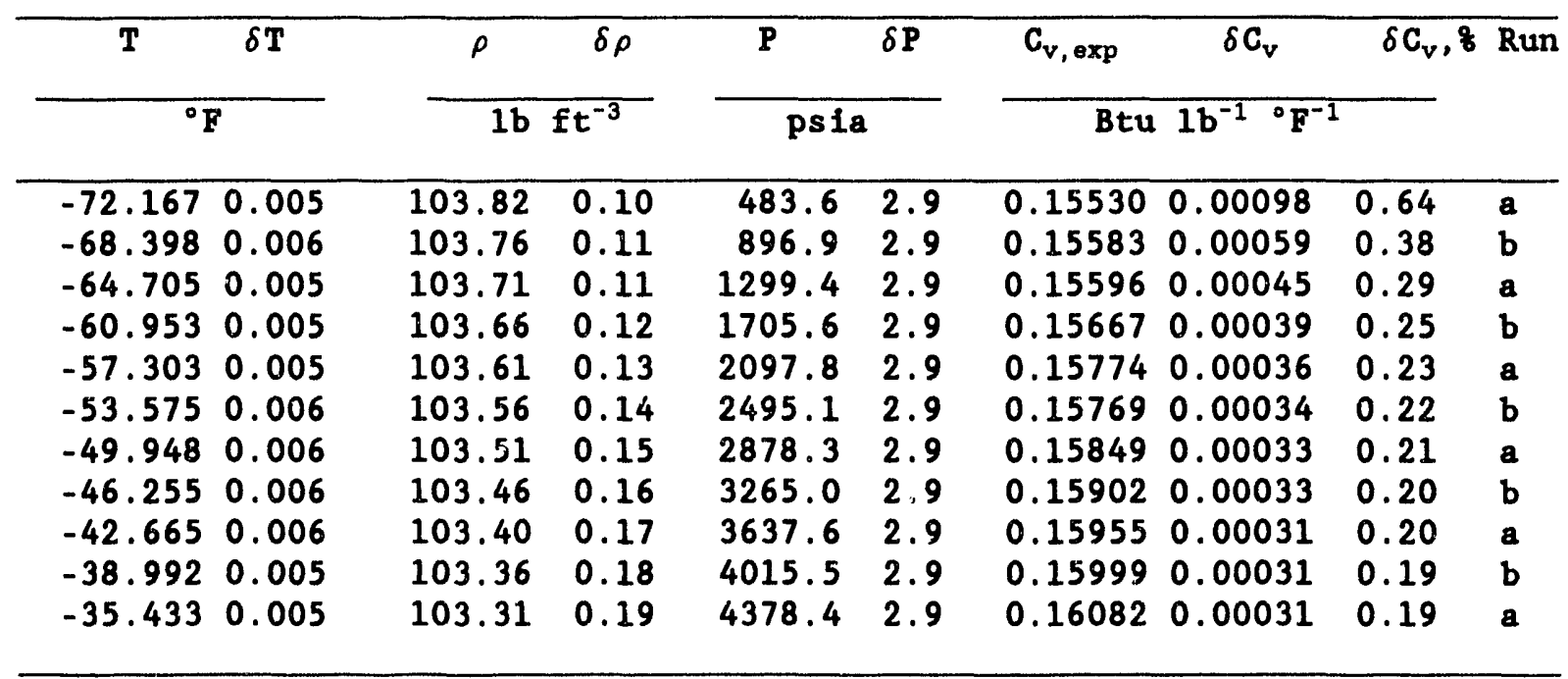


Table $30(a)$. Experimental liquid heat capacity data for HCFC-123.

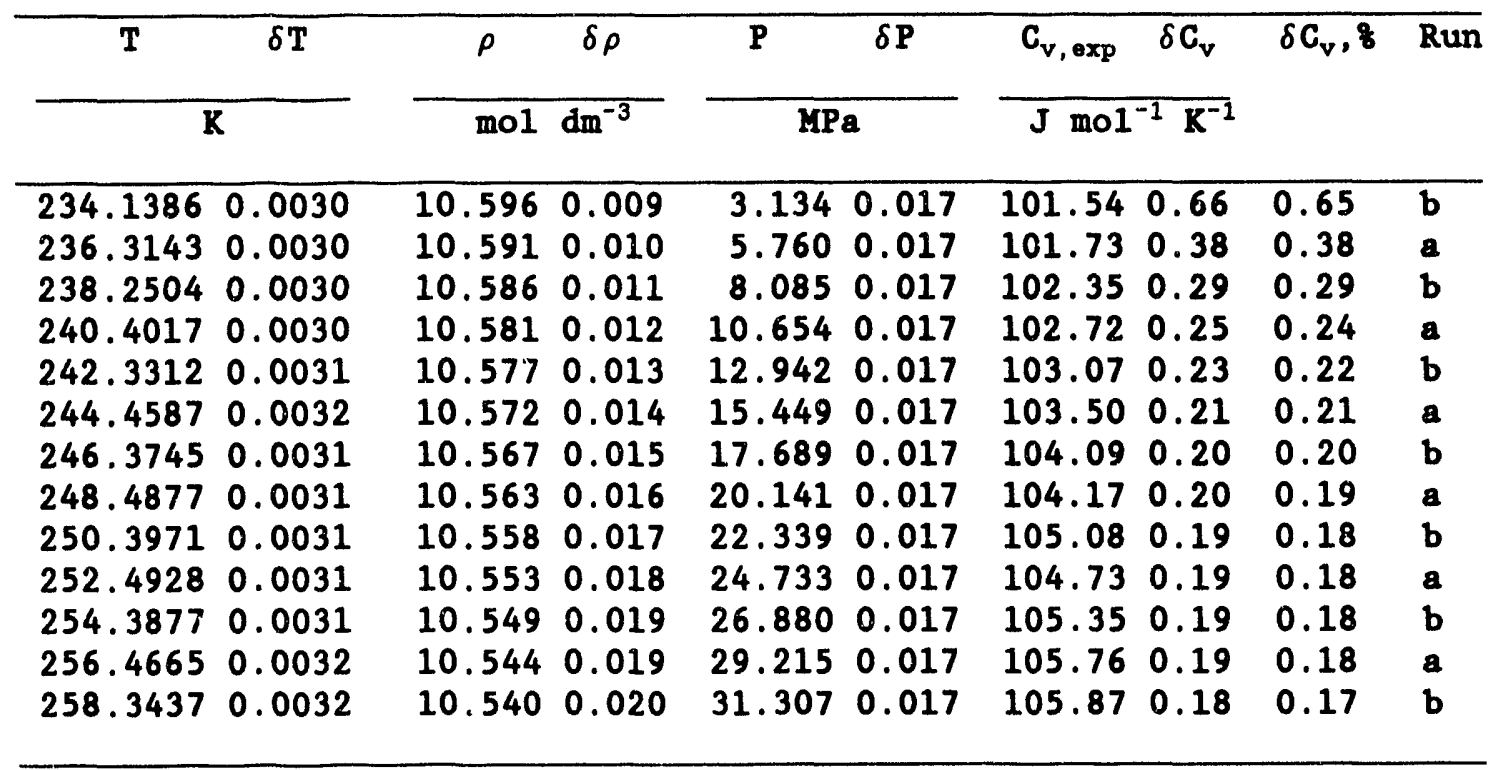

Table $30(b)$. Experimental liquid heat capacity data for HCFC-123.

\begin{tabular}{|c|c|c|c|c|c|c|c|c|c|}
\hline $\mathbf{T}$ & $\delta \mathbf{T}$ & $\rho$ & $\delta \rho$ & $\mathbf{P}$ & $\delta \mathbf{P}$ & $C_{v, \exp }$ & $\delta \mathrm{C}_{\mathrm{v}}$ & $\delta \mathrm{C}_{\mathrm{v}}, 8$ & Run \\
\hline \multicolumn{2}{|c|}{${ }^{\circ} \mathrm{F}$} & \multicolumn{2}{|c|}{$1 b \mathrm{ft}^{-3}$} & \multicolumn{2}{|c|}{ psia } & \multicolumn{4}{|c|}{ Btu $1 b^{-1} \cdot{ }^{\circ} F^{-1}$} \\
\hline $\begin{array}{r}-38.221 \\
-34.304 \\
-30.819 \\
-26.947 \\
-23.474 \\
-19.644 \\
-16.196 \\
-12.392 \\
-8.955 \\
-5.183 \\
-1.772 \\
1.970 \\
5.349\end{array}$ & $\begin{array}{l}0.005 \\
0.005 \\
0.005 \\
0.005 \\
0.006 \\
0.006 \\
0.006 \\
0.006 \\
0.006 \\
0.006 \\
0.006 \\
0.006 \\
0.006\end{array}$ & $\begin{array}{l}101.16 \\
101.11 \\
101.07 \\
101.02 \\
100.98 \\
100.93 \\
100.88 \\
100.85 \\
100.80 \\
100.75 \\
100.71 \\
100.66 \\
100.63\end{array}$ & $\begin{array}{l}0.09 \\
0.10 \\
0.11 \\
0.11 \\
0.12 \\
0.13 \\
0.14 \\
0.15 \\
0.16 \\
0.17 \\
0.18 \\
0.18 \\
0.19\end{array}$ & $\begin{array}{r}454.5 \\
835.4 \\
1172.6 \\
1545.2 \\
1877.1 \\
2240.7 \\
2565.6 \\
2921.2 \\
3240.0 \\
3587.2 \\
3898.6 \\
4237.3 \\
4540.7\end{array}$ & $\begin{array}{l}2.5 \\
2.5 \\
2.5 \\
2.5 \\
2.5 \\
2.5 \\
2.5 \\
2.5 \\
2.5 \\
2.5 \\
2.5 \\
2.5 \\
2.5\end{array}$ & $\begin{array}{l}0.15869 \\
0.15899 \\
0.15996 \\
0.16054 \\
0.16108 \\
0.16175 \\
0.16268 \\
0.16280 \\
0.16422 \\
0.16368 \\
0.16465 \\
0.16529 \\
0.16546\end{array}$ & $\begin{array}{l}0.00103 \\
0.00059 \\
0.00045 \\
0.00039 \\
0.00036 \\
0.00033 \\
0.00031 \\
0.00031 \\
0.00030 \\
0.00030 \\
0.00030 \\
0.00030 \\
0.00028\end{array}$ & $\begin{array}{l}0.65 \\
0.38 \\
0.29 \\
0.24 \\
0.22 \\
0.21 \\
0.20 \\
0.19 \\
0.18 \\
0.18 \\
0.18 \\
0.18 \\
0.17\end{array}$ & $\begin{array}{l}\text { b } \\
a \\
b \\
a \\
b \\
a \\
b \\
a \\
b \\
a \\
b \\
a \\
b\end{array}$ \\
\hline
\end{tabular}


Table 31(a). Experimental two-phase heat capacity data for HCFC-123.

\begin{tabular}{|c|c|c|c|c|c|c|c|c|c|c|}
\hline $\mathrm{T}$ & $\delta \mathrm{T}$ & $\rho_{\sigma}$ & $\mathbf{P}_{\sigma}$ & $\mathrm{C}_{\mathrm{v}}{ }^{(2)}$ & $\delta C_{v}$ & $00 \mathrm{v}$ & $8 \quad \mathrm{C}_{\sigma}$ & c & $\mathrm{s}_{\sigma}$ & 8 \\
\hline$\overline{1}$ & 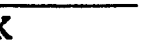 & $\mathrm{mol} \mathrm{dm}^{-3}$ & $\mathbf{P a}$ & & & 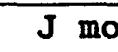 & 14 & & & \\
\hline 166.9425 & 0.0030 & 11.419 & 0.0001 & 137.83 & 0.11 & 0.08 & 137.82 & 0.11 & 0.08 & c \\
\hline 171.0998 & 0.0030 & 11.368 & 0.0001 & 137.91 & 0.10 & 0.08 & 137.90 & 0.11 & 0.08 & c \\
\hline 171.7387 & 30 & 11.360 & 0.0002 & 138.08 & 0.14 & 0.10 & 138.07 & 0.14 & 0.10 & $\mathbf{a}$ \\
\hline 174. & & 11.320 & 0.0 & 138.29 & 0.13 & 0.10 & 138.28 & 0.13 & 0.10 & $\mathbf{a}$ \\
\hline 175.2230 & 0. & 11. & 0.0002 & 138.32 & 0.11 & 0.08 & 138.31 & 0.11 & 0.08 & c \\
\hline 178.0067 & 0.0033 & 11.281 & 0.0003 & 138.63 & 0.16 & 0.11 & 138.62 & 0.16 & 0.11 & $\mathbf{a}$ \\
\hline 179.3247 & 0.0030 & 11.264 & 0.0004 & 138.46 & 0.11 & 0.08 & 138.45 & 0.11 & 0.08 & c \\
\hline 181.5964 & 0.0030 & 11.235 & 0.0005 & 139.03 & 0.11 & 0.08 & 139.01 & 0.11 & 0.08 & $\mathbf{a}$ \\
\hline 183.3926 & 0.0030 & 11.212 & 0.0006 & 138.84 & 0.11 & 0.08 & 138.82 & 0.11 & 0.08 & c \\
\hline 185.6505 & 0.0030 & 11.183 & 0.0008 & 139.87 & 0.11 & 0.08 & 139.85 & 0.11 & 0.08 & $\mathbf{a}$ \\
\hline 187. & 0.0 & 11. & 0.0009 & 139.19 & 0.11 & 0.08 & 139.17 & 0.11 & 0.08 & c \\
\hline 189. & 0. & 11. & 0. & 139.82 & 1 & 0 & 139.79 & 0.11 & 08 & $\mathbf{a}$ \\
\hline 191. & 0.1 & 11 & 0.0 & 139.84 & 0.12 & 0.08 & 139.81 & 0.12 & 0.08 & c \\
\hline 193.6829 & 0.0031 & 11.080 & 0.0017 & 139.94 & 0.11 & 0.08 & 139.90 & 0.11 & 0.08 & 8 \\
\hline 195.4422 & 0.0031 & 11.057 & 0.0020 & 140.06 & 0.11 & 0.08 & 140.02 & 0.11 & 0.08 & c \\
\hline 197.6566 & 0.0030 & 11.028 & 0.0025 & 140.36 & 0.11 & 0.08 & 140.31 & 0.11 & 0.08 & 8 \\
\hline 81 & 30 & 11.005 & 0.0029 & 140.49 & 0.12 & 0.08 & 140.43 & 0.12 & 0.08 & $\mathbf{c}$ \\
\hline 201.5701 & 0.0031 & 10.977 & 0.0035 & 146.12 & 0.12 & 0.09 & 146.05 & 0.13 & 0.09 & $\mathbf{a}$ \\
\hline 203.3431 & 0.0032 & 10.953 & 0.0 & 140.57 & 0.12 & 0.08 & 140.50 & 0.12 & 0.09 & c \\
\hline 205. & $0 . c$ & 10 & 0. & .42 & 2 & 0. & 141.34 & 0.12 & 9 & $\mathbf{b}$ \\
\hline 207. & 0.0 & 10 & 0.0 & 141.46 & 0.12 & 0.08 & 141.37 & 0.12 & 0.09 & c \\
\hline 209.3703 & 0.0031 & 10.873 & 0.0068 & 141.67 & 0.12 & 0.09 & 141.57 & 0.13 & 0.09 & b \\
\hline 211.1434 & 0.0031 & 10.849 & 0.0078 & 142.39 & 0.12 & 0.08 & 142.27 & 0.12 & 0.09 & $c$ \\
\hline 04 & 0.0 & 10 & 92 & .56 & 0.13 & 0.09 & 142.43 & 0.13 & 0.09 & $t$ \\
\hline 215.0115 & 0.0030 & 10 & 06 & 142.20 & 0.12 & 0.08 & 142.07 & 0.13 & 0.09 & c \\
\hline 217. & 0.0031 & 10. & 0.0 & 142.66 & 0.12 & 0.08 & 142.51 & 0.13 & 0.09 & $\mathbf{b}$ \\
\hline 218. & 0.0 & 10 & 0. & .73 & 0.12 & 0.08 & .57 & 0.13 & 0.09 & c \\
\hline 220. & 0.0 & 10 & 164 & 143.25 & 0.13 & 0.09 & 143.07 & 0.15 & 0.10 & $\mathbf{b}$ \\
\hline 222.6798 & 0.0 & 10. & 0.0186 & 143.31 & 0.12 & 0.08 & 143.12 & 0.14 & 0.09 & c \\
\hline 224.7490 & 0.0033 & 10.665 & 0.0215 & 143.89 & 0.13 & 0.09 & 143.68 & 0.15 & 0.11 & b \\
\hline 226.4838 & 0.0031 & 10.641 & 0.0243 & 143.84 & 0.13 & 0.09 & 143.62 & 0.15 & 0.10 & c \\
\hline 228.5416 & 0.0034 & 10.612 & 0.0279 & 145.16 & 0.14 & 0.10 & 144.92 & 0.16 & 0.11 & b \\
\hline 230. & 0.0 & 10 & 0.0 & 144.44 & 0.12 & 0.09 & 144.19 & 0.16 & 0.11 & c \\
\hline 232.3220 & 0.0031 & 10.560 & 0. & 144.97 & 0.13 & 0.09 & 144.70 & 0.16 & 0.11 & b \\
\hline 234.0181 & 0.0034 & 10. & 0.0 & 145.03 & 0.14 & 0.10 & 144.74 & 0.18 & 0.12 & d \\
\hline 236. & 0.0 & 10 & 0. & 144.90 & 0.13 & 0.09 & 144.59 & 0.17 & 0.12 & b \\
\hline 237.7526 & 0.0 & 10 & 0. & 145.33 & 0.13 & 0 & 145.01 & 0.18 & 0.13 & d \\
\hline 239.7937 & 0.0 & 10 & 0. & 145.45 & 0.13 & 0.09 & 145.11 & 0.19 & 0.13 & b \\
\hline 241.4666 & 0.0 & 10 & 0.0 & 145.99 & 0.14 & 0.10 & 145.64 & 0.20 & 0.14 & d \\
\hline 243.5063 & 0.0032 & 10.402 & 0.0712 & 146.23 & 0.13 & 0.09 & 145.86 & 0.20 & 0.14 & b \\
\hline 245.1696 & 0.0031 & 10.378 & 0.0785 & 145.91 & 0.13 & 0.09 & 145.53 & 0.21 & 0.14 & d \\
\hline 247.1947 & 0.003 & 10.349 & 0.0881 & 147.01 & 0.14 & 0.10 & 146.62 & 0.22 & 0.15 & b \\
\hline 248.8370 & 0.0032 & 10.325 & 0.0966 & 147.03 & 0.14 & 0.10 & 146.63 & 0.23 & 0.16 & d \\
\hline 250.8710 & 0.0032 & 10.296 & 0.1080 & 147.63 & 0.1 .5 & 0.10 & 147.21 & 0.24 & 0.16 & b \\
\hline 252.5090 & 0.0032 & 10.272 & 0.1181 & 147.37 & 0.14 & 0.09 & 146.95 & 0.24 & 0.16 & d \\
\hline 254.5302 & 0.0032 & 10.243 & 0.1316 & $147\ulcorner 6$ & 0.15 & 0.10 & 147.13 & 0.25 & 0.17 & $\mathbf{b}$ \\
\hline 56 & 1031 & 9 & 0.1432 & 47 & 0.16 & 1 & 147 & 0.27 & 0.18 & d \\
\hline
\end{tabular}


Table 31 (a)(continued). Experimental two-phase heat capacity data for HCFC-123.

\begin{tabular}{|c|c|c|c|c|c|c|c|c|c|c|}
\hline$T$ & $\overline{\delta T}$ & & & $C_{v}{ }^{(2)}$ & $\delta C_{v}{ }^{(2)}$ & $\delta \mathrm{C}$ & $\begin{array}{ll}C_{0} \\
\end{array}$ & $\delta \mathrm{C}_{0}$ & $\delta \mathrm{C}_{\sigma}, \mathrm{z}$ & $8 \mathrm{Rz}$ \\
\hline$\overline{\mathbf{X}}$ & & $01 \mathrm{dm}^{-3}$ & MPa & & & $\bar{J}$ & $\mathrm{~K}^{-1}$ & & & \\
\hline $\begin{array}{l}258.1638 \\
259.7729 \\
261.7865 \\
263.3859 \\
265.3879 \\
266.9878 \\
268.9728 \\
270.5732 \\
272.5468 \\
274.1380 \\
276.1054 \\
277.6859 \\
279.6487 \\
281.2308 \\
283.1806 \\
284.7545 \\
286.6920 \\
288.2690 \\
290.1942 \\
291.7702 \\
293.6878 \\
295.2590 \\
297.1620 \\
298.7470 \\
300.6368 \\
302.2147 \\
304.0893 \\
307.5811\end{array}$ & $\begin{array}{l}0.0031 \\
0.0034 \\
0.0032 \\
0.0032 \\
0.0031 \\
0.0031 \\
0.0034 \\
0.0031 \\
0.0032 \\
0.0032 \\
0.0031 \\
0.0031 \\
0.0036 \\
0.0032 \\
0.0032 \\
0.0033 \\
0.0033 \\
0.0032 \\
0.0033 \\
0.0033 \\
0.0035 \\
0.0032 \\
0.0033 \\
0.0032 \\
0.0038 \\
0.0035 \\
0.0034 \\
0.0035\end{array}$ & $\begin{array}{l}10.189 \\
10.165 \\
10.135 \\
10.112 \\
10.082 \\
10.057 \\
10.027 \\
10.003 \\
9.973 \\
9.949 \\
9.918 \\
9.894 \\
9.863 \\
9.839 \\
9.808 \\
9.783 \\
9.753 \\
9.728 \\
9.697 \\
9.672 \\
9.641 \\
9.615 \\
9.584 \\
9.558 \\
9.527 \\
9.501 \\
9.470 \\
9.411\end{array}$ & $\begin{array}{l}0.1590 \\
0.1726 \\
0.1910 \\
0.2067 \\
0.2279 \\
0.2460 \\
0.2702 \\
0.2911 \\
0.3187 \\
0.3424 \\
0.3737 \\
0.4006 \\
0.4361 \\
0.4665 \\
0.5063 \\
0.5404 \\
0.5850 \\
0.6233 \\
0.6729 \\
0.7158 \\
0.7709 \\
0.8185 \\
0.8793 \\
0.9326 \\
0.9994 \\
1.0581 \\
1.1313 \\
1.2783\end{array}$ & $\begin{array}{l}148.03 \\
148.83 \\
139.03 \\
139.84 \\
149.60 \\
150.01 \\
151.07 \\
150.28 \\
151.61 \\
150.90 \\
151.86 \\
152.35 \\
152.03 \\
152.98 \\
152.50 \\
153.09 \\
153.25 \\
153.80 \\
154.00 \\
154.68 \\
154.09 \\
155.20 \\
154.61 \\
155.27 \\
155.04 \\
156.55 \\
155.48 \\
151.27\end{array}$ & $\begin{array}{l}0.140 \\
0.170 \\
5.99 \\
5.96 \\
0.140 \\
0.140 \\
0.150 \\
0.140 \\
0.15 \\
0.140 \\
0.14 \\
0.14 \\
0.16 \\
0.15 \\
0.15 \\
0.15 \\
0.15 \\
0.15 \\
0.15 \\
0.15 \\
0.16 \\
0.15 \\
0.15 \\
0.15 \\
0.17 \\
0.17 \\
0.16 \\
0.16\end{array}$ & $\begin{array}{l}0.10 \\
0.11 \\
4.31 \\
4.27 \\
0.09 \\
0.09 \\
0.10 \\
0.09 \\
0.10 \\
0.10 \\
0.09 \\
0.09 \\
0.11 \\
0.10 \\
0.10 \\
0.10 \\
0.10 \\
0.10 \\
0.10 \\
0.10 \\
0.11 \\
0.10 \\
0.10 \\
0.10 \\
0.11 \\
0.11 \\
0.10 \\
0.11\end{array}$ & $\begin{array}{l}147.61 \\
148.41 \\
138.62 \\
139.42 \\
149.19 \\
149.62 \\
150.70 \\
149.95 \\
151.31 \\
150.64 \\
151.65 \\
152.19 \\
151.94 \\
152.95 \\
152.57 \\
153.23 \\
153.51 \\
154.15 \\
154.48 \\
155.28 \\
154.86 \\
156.11 \\
155.71 \\
156.54 \\
156.52 \\
158.24 \\
157.41 \\
153.73\end{array}$ & $\begin{array}{l}0.27 \\
0.29 \\
5.99 \\
5.97 \\
0.29 \\
0.30 \\
0.31 \\
0.31 \\
0.32 \\
0.32 \\
0.32 \\
0.33 \\
0.34 \\
0.33 \\
0.33 \\
0.33 \\
0.33 \\
0.33 \\
0.33 \\
0.32 \\
0.32 \\
0.31 \\
0.31 \\
0.30 \\
0.31 \\
0.30 \\
0.29 \\
0.30\end{array}$ & $\begin{array}{l}0.18 \\
0.19 \\
4.32 \\
4.28 \\
0.20 \\
0.20 \\
0.21 \\
0.21 \\
0.21 \\
0.21 \\
0.21 \\
0.21 \\
0.22 \\
0.22 \\
0.22 \\
0.22 \\
0.22 \\
0.21 \\
0.21 \\
0.21 \\
0.21 \\
0.20 \\
0.20 \\
0.19 \\
0.20 \\
0.19 \\
0.19 \\
0.20\end{array}$ & $\begin{array}{l}b \\
d \\
b \\
d \\
b \\
d \\
b \\
d \\
b \\
d \\
b \\
d \\
b \\
d \\
b \\
d \\
b \\
d \\
b \\
d \\
b \\
d \\
b \\
d \\
b \\
d \\
b \\
b\end{array}$ \\
\hline
\end{tabular}


Table 31(b). Experimental two-phase heat capacity data for HCFC-123.

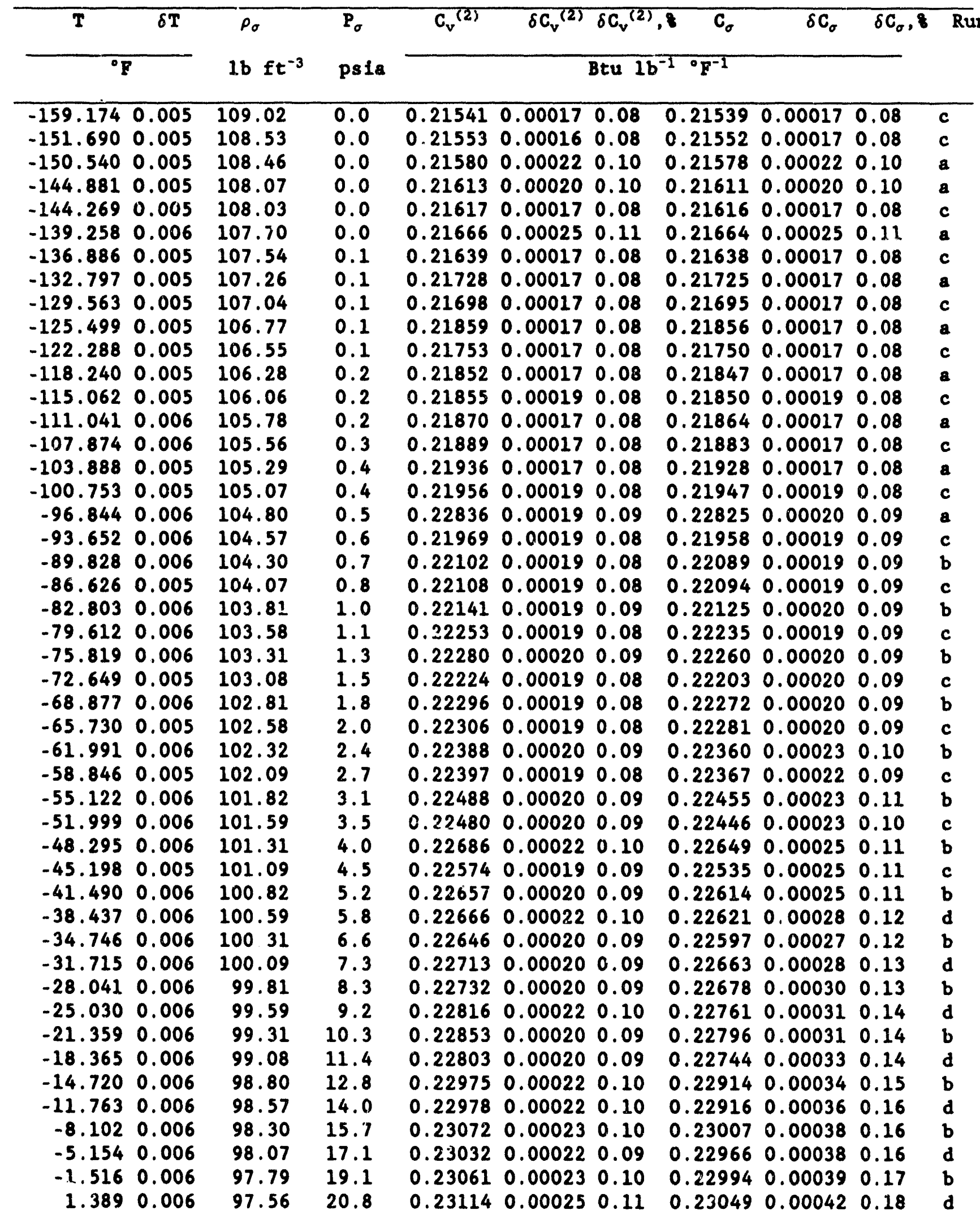


Table $31(b)$ (continued). Experimental two-phase heat capacity data for HCFC-123.

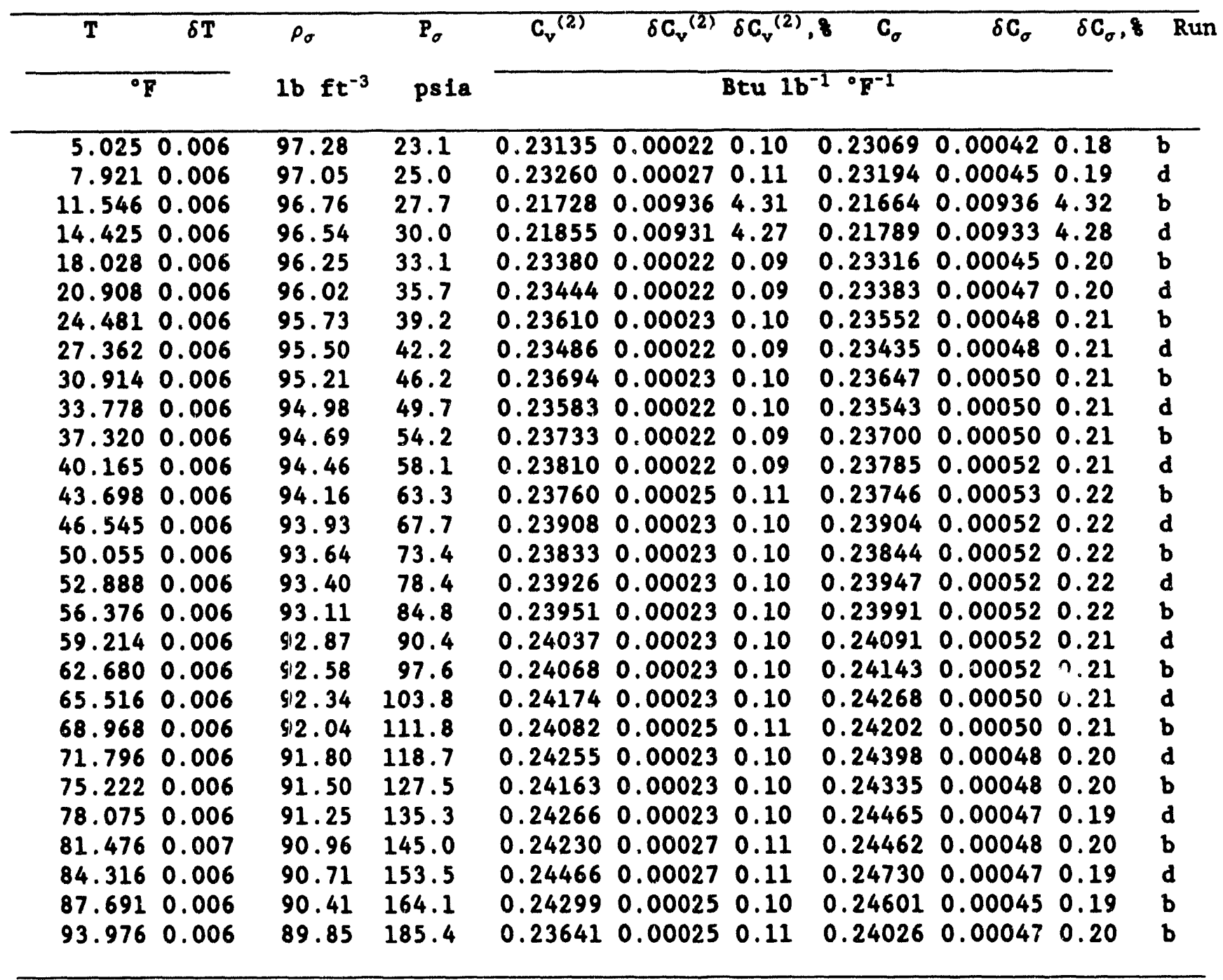


Table $32(a)$. Experimental two-phase heat capacity data for HCFC-123.

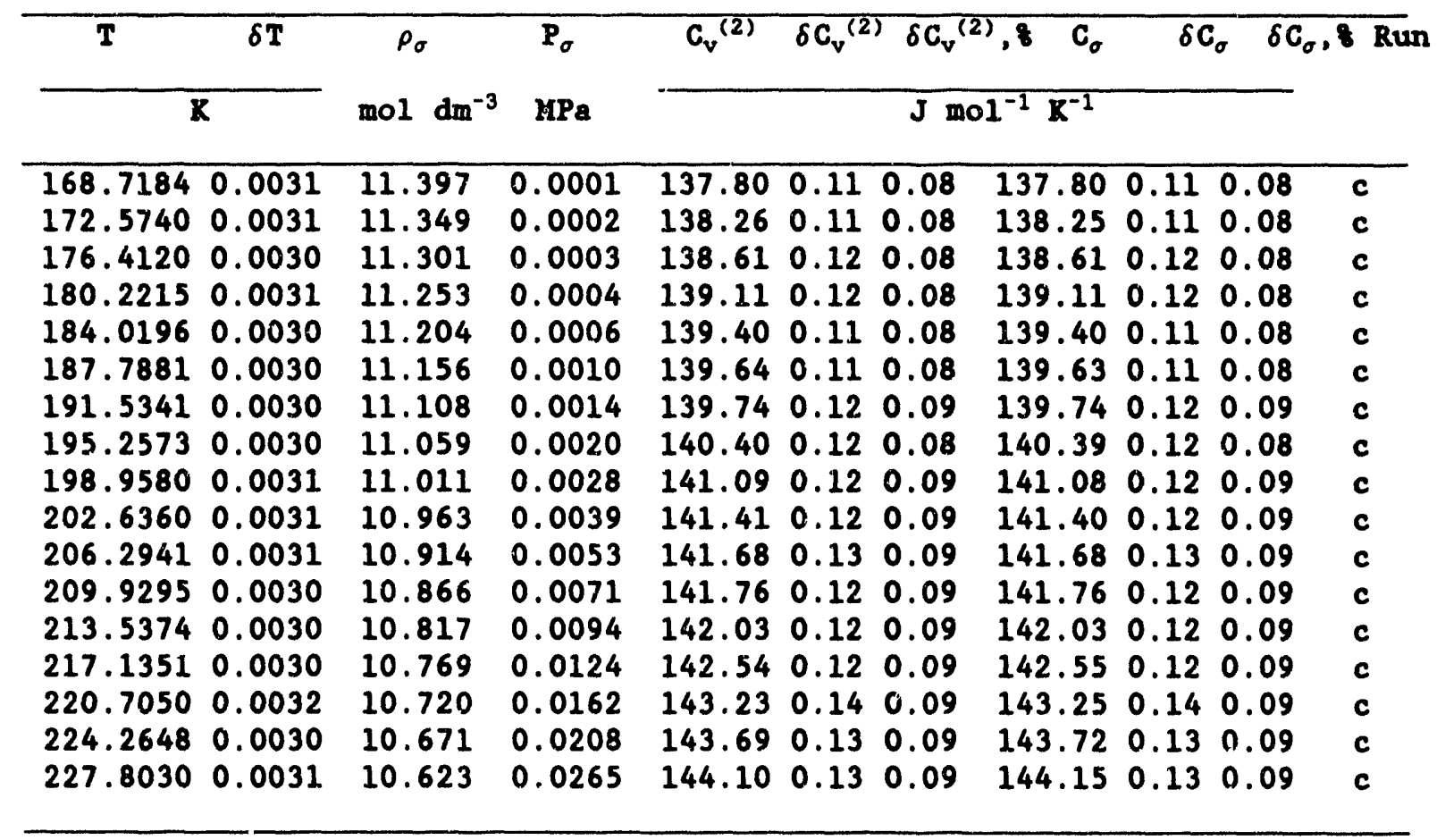

Table 32(b). Experimental two-phase heat capacity data for HCFC-123.

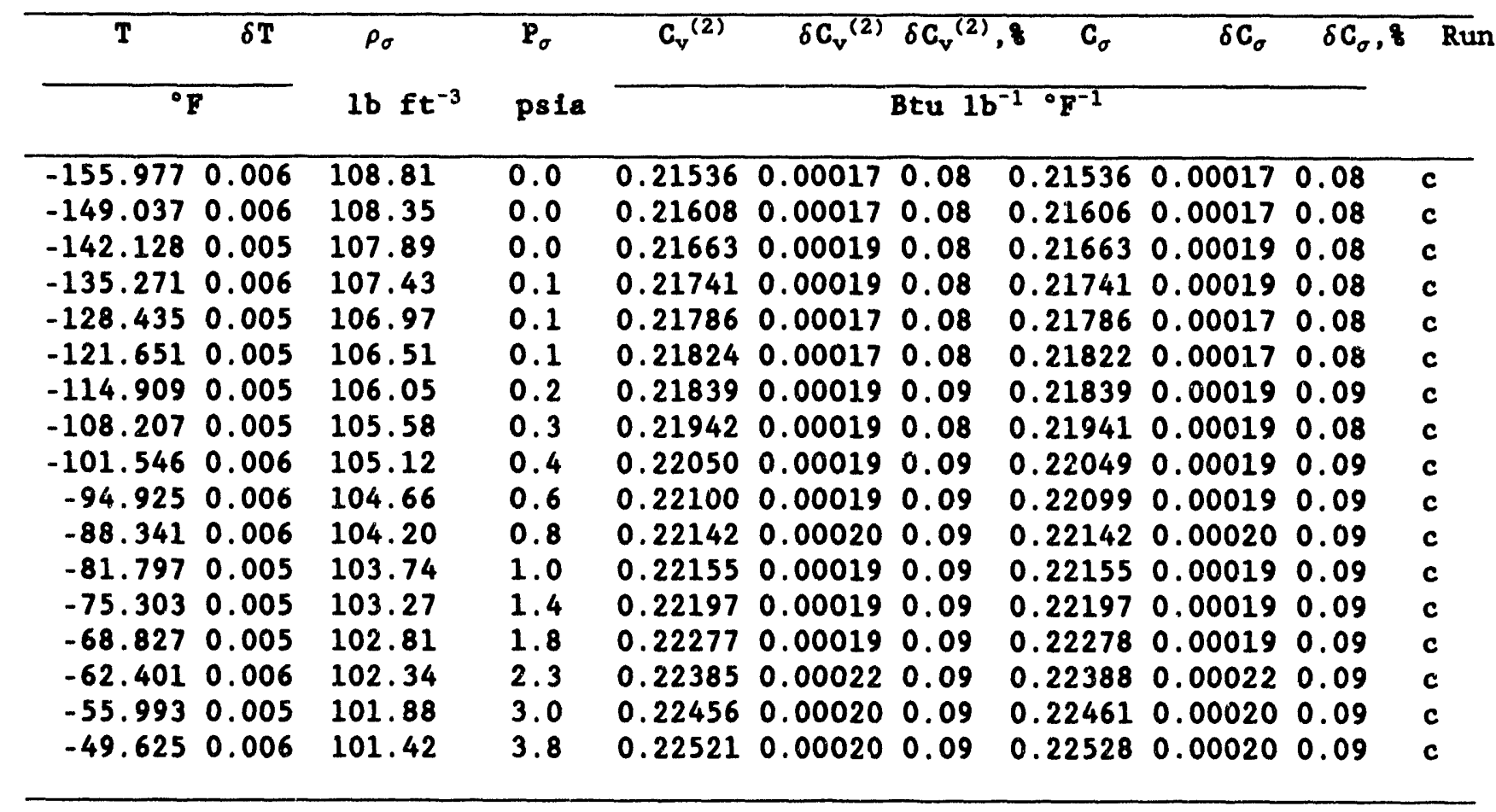



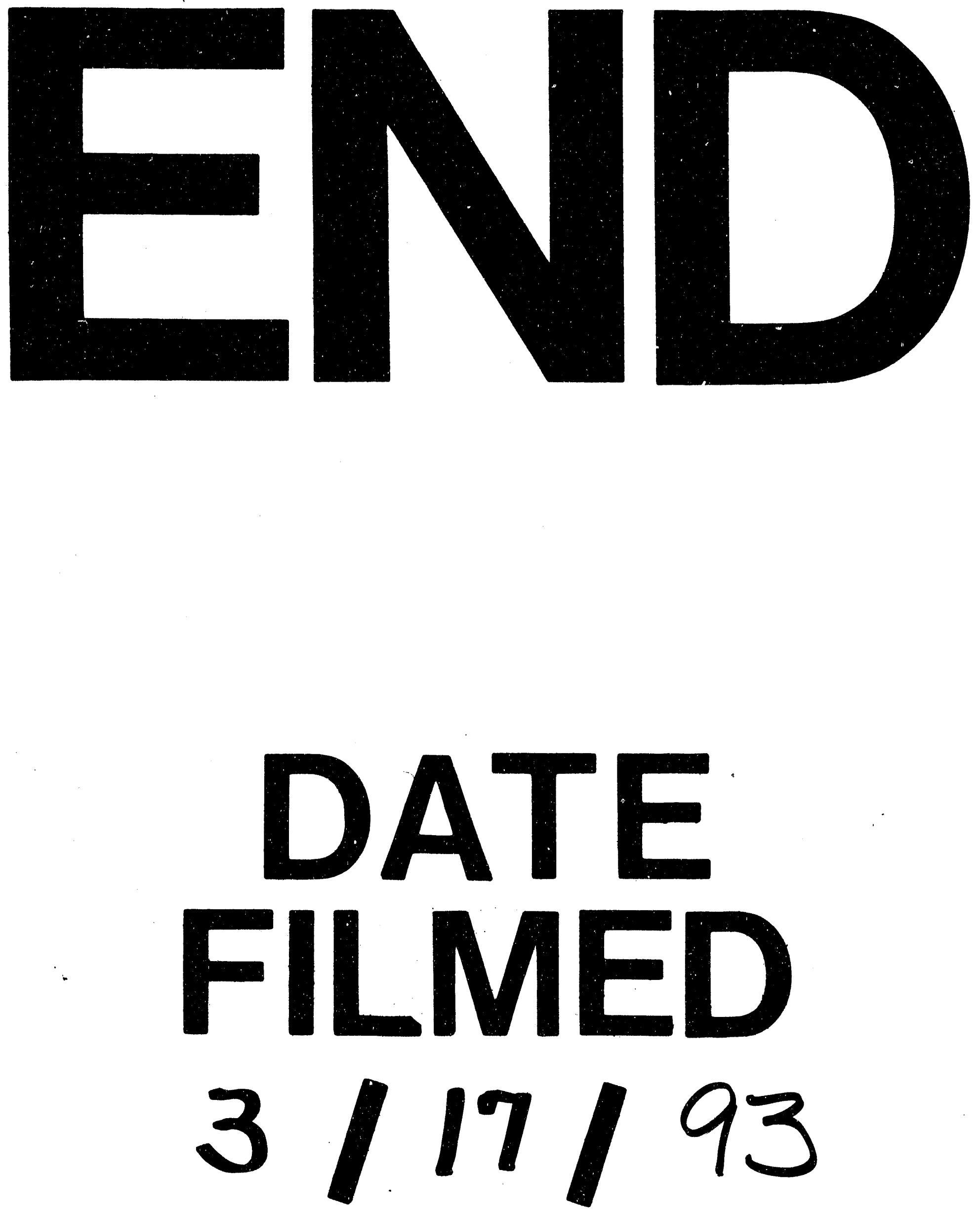
